\title{
Periodic Solutions of a Many-Rotator Problem in the Plane. II. Analysis of Various Motions
}

F CALOGERO †‡, J-P FRANÇOISE * and MSOMMACAL *

† Dipartimento di Fisica, Università di Roma "La Sapienza", 00185 Roma, Italy

E-mail: francesco.calogero@uniroma1.it

¥ Istituto Nazionale di Fisica Nucleare, Sezione di Roma, Italy

E-mail: francesco.calogero@roma1.infn.it

* GSIB, 175 Rue du Chevaleret, Université Paris VI, 75013 Paris, France

E-mail: jpf@ccr.jussieu.fr

\# Dipartimento di Fisica, Università di Roma "La Sapienza", 00185 Roma, Italy

E-mail:sommacal@sissa.it

Received May 13, 2002; Accepted August 2, 2002

\begin{abstract}
Various solutions are displayed and analyzed (both analytically and numerically) of a recently-introduced many-body problem in the plane which includes both integrable and nonintegrable cases (depending on the values of the coupling constants); in particular the origin of certain periodic behaviors is explained. The light thereby shone on the connection among integrability and analyticity in (complex) time, as well as on the emergence of a chaotic behavior (in the guise of a sensitive dependance on the initial data) not associated with any local exponential divergence of trajectories in phase space, might illuminate interesting phenomena of more general validity than for the particular model considered herein.
\end{abstract}

\section{Introduction}

Recently it was pointed out $[1,2]$ that the $N$-body problem in the plane characterized by the following Newtonian equations of motion possesses lots of completely periodic solutions:

$$
\begin{aligned}
\ddot{\vec{r}}_{n}= & \omega \hat{k} \wedge \dot{\vec{r}}_{n}+2 \sum_{m=1 ; m \neq n}^{N}\left(r_{n m}\right)^{-2}\left(\alpha_{n m}+\alpha_{n m}^{\prime} \hat{k} \wedge\right) \\
& \times\left[\dot{\vec{r}}_{n}\left(\dot{\vec{r}}_{m} \cdot \vec{r}_{n m}\right)+\dot{\vec{r}}_{m}\left(\dot{\vec{r}}_{n} \cdot \vec{r}_{n m}\right)-\vec{r}_{n m}\left(\dot{\vec{r}}_{n} \cdot \dot{\vec{r}}_{m}\right)\right] .
\end{aligned}
$$

Here and below the subscripted indices run from 1 to $N$ (unless otherwise indicated), the number of moving particles $N$ is a positive integer, the $N$ two-vectors $\vec{r}_{n} \equiv \vec{r}_{n}(t)$ identify 
the positions of the moving point-particles in a plane which for notational convenience we imagine immersed in ordinary three-dimensional space, so that $\vec{r}_{n} \equiv\left(x_{n}, y_{n}, 0\right) ; \hat{k}$ is the unit three-vector orthogonal to that plane, $\hat{k} \equiv(0,0,1)$, so that $\hat{k} \wedge \vec{r}_{n} \equiv\left(-y_{n}, x_{n}, 0\right)$;

$$
\vec{r}_{n m} \equiv \vec{r}_{n}-\vec{r}_{m}, \quad r_{n m}^{2}=r_{n}^{2}+r_{m}^{2}-2 \vec{r}_{n} \cdot \vec{r}_{m}
$$

superimposed dots denote of course time derivatives; $\omega$ is a real-indeed, without loss of generality, positive - constant, $\omega>0$, which sets the time scale, and to which we associate the period

$$
T=2 \pi / \omega
$$

(when discussing below specific examples we will conveniently set $\omega=2 \pi$, so that $T=1$; but for the moment let us not do that); and $\alpha_{n m}$ and $\alpha_{n m}^{\prime}$ are real "coupling constants". Note that this $N$-body model in the plane is invariant under both translations and rotations; as we shall see below, it is Hamiltonian; it features one-body and two-body velocitydependent forces; when the latter are missing - namely, when all the two-body coupling constants $\alpha_{n m}, \alpha_{n m}^{\prime}$ vanish,

$$
\alpha_{n m}=\alpha_{n m}^{\prime}=0,
$$

it represents the physical situation of $N$ electrically charged particles moving in a plane under the influence of a constant magnetic field orthogonal to that plane ("cyclotron"). In such a case of course every particle moves uniformly, with the same period $T$, see (1.2), on a circular trajectory the center $\vec{c}_{n}$ and radius $\rho_{n}$ of which are determined by the initial data (position and velocity) of each particle:

$$
\begin{aligned}
& \vec{r}_{n}(t)=\vec{c}_{n}+\vec{\rho}_{n} \sin (\omega t)-\hat{k} \wedge \vec{\rho}_{n} \cos (\omega t), \\
& \vec{c}_{n}=\vec{r}_{n}(0)+\hat{k} \wedge \vec{\rho}_{n}, \quad \vec{\rho}_{n}=\dot{\vec{r}}_{n}(0) / \omega .
\end{aligned}
$$

Let us begin by tersely reviewing previous findings [1, 2]. First of all we note that it is convenient to identify the physical plane in which the motion takes place with the complex plane, via the relation

$$
\vec{r}_{n} \equiv\left(x_{n}, y_{n}, 0\right) \Leftrightarrow z_{n} \equiv x_{n}+i y_{n},
$$

whereby the real Newtonian equations of motion in the plane (1.1) become the following equations determining the motion of the $N$ points $z_{n} \equiv z_{n}(t)$ in the complex $z$-plane:

$$
\ddot{z}_{n}=i \omega \dot{z}_{n}+2 \sum_{m=1 ; m \neq n}^{N} a_{n m} \dot{z}_{n} \dot{z}_{m} /\left(z_{n}-z_{m}\right),
$$

with

$$
a_{n m}=\alpha_{n m}+i \alpha_{n m}^{\prime} .
$$

Hereafter we always use this avatar, (1.5), of the equations of motion (1.1), and we moreover exploit the following key observation $[3,4,1,2]$ : via the change of (independent) variable

$$
\underline{z}(t)=\underline{\zeta}(\tau), \quad \tau=[\exp (i \omega t)-1] /(i \omega),
$$


the system (1.5) becomes

$$
\zeta_{n}^{\prime \prime}=2 \sum_{m=1 ; m \neq n}^{N} a_{n m} \zeta_{n}^{\prime} \zeta_{m}^{\prime} /\left(\zeta_{n}-\zeta_{m}\right)
$$

Here and below the underlined notation indicates an $N$-vector, say $\underline{z} \equiv\left(z_{1}, \ldots, z_{N}\right)$, $\underline{\zeta} \equiv\left(\zeta_{1}, \ldots, \zeta_{N}\right)$ and so on, and the primes denote of course differentiations with respect to the independent variable $\tau$. Note that the constant $\omega$ has completely disappeared from (1.7); nor does it feature in the relations among the initial data for (1.5) and (1.7), which read simply

$$
\underline{z}(0)=\underline{\zeta}(0), \quad \underline{\dot{z}}(0)=\underline{\zeta^{\prime}}(0) .
$$

This of course entails that, to obtain the solution $\underline{z}(t)$ of (1.5) corresponding to a given set of initial data $\underline{z}(0), \underline{\dot{z}}(0)$, one can solve (1.7) with the same set of initial data, see (1.8), thereby determine $\underline{\zeta}(\tau)$, and then use (1.6) to obtain $\underline{z}(t)$ (hence as well, via (1.4), the solution of the initial-value problem for (1.1)). This possibility - to infer the behavior of the evolution in the real time variable $t$ of the solutions $\underline{z}(t)$ of (1.5) (namely as well of the solutions of the physical many-body problem in the plane (1.1) ) from the properties of the solutions $\underline{\zeta}(\tau)$ of (1.7) as functions of the complex variable $\tau$ - is, as we will see, the main tool of our investigation. Indeed, when the real time variable $t$ evolves over one period $T$ - say, from $t=0$ to $t=T$ - the complex time-like variable $\tau$ goes from $\tau=0$ back to $\tau=0$ by traveling counter-clock-wise - see (1.6) - on the circular contour $\tilde{C}$ centered in the complex $\tau$-plane at $i / \omega$ and having radius $1 / \omega$. Hence whenever all the functions $\zeta_{n}(\tau)$ - obtained as solutions of (1.7) - are holomorphic, as functions of the complex variable $\tau$, inside that circular contour $\tilde{C}$ - or, more precisely, in the (closed) circular disk $C$ defined by that contour $\tilde{C}$ - the corresponding functions $z_{n}(t)$ - namely the corresponding solutions of (1.5) - are nonsingular and completely periodic in the real time variable $t$, with period $T$, see (1.2). (This also entails that, if the only singularities of a solution $\underline{\zeta}(\tau)$ of (1.7) inside the disk $C$ are a finite number of rational branch points, then the corresponding solution $\underline{z}(t)$ of (1.5) is again completely periodic in the real time $t$, albeit with a larger period which is then an entire multiple of $T$ - we shall discuss in detail this important point below).

Now we note that all solutions $\underline{\zeta}(\tau)$ of (1.7), corresponding to arbitrary initial data $\underline{\zeta}(0), \underline{\zeta}^{\prime}(0)$ (with the only restriction that these data be nonsingular, namely $\left|\zeta_{n}^{\prime}(0)\right|<\infty$ and $\zeta_{n}(0) \neq \zeta_{m}(0)$, see the right-hand side of (1.7)), yield solutions $\underline{\zeta}(\tau)$ which are holomorphic in the neighborhood of $\tau=0$, as implied by the standard theorem which guarantees the existence, uniqueness and analyticity of the solutions of analytic ODEs, in a sufficiently small circular disk $D$ centered in the complex $\tau$-plane at the origin, $\tau=0$, where the initial data defining the solution are given. The size of this disk $D$ is determined by the location of the singularity of $\zeta(\tau)$ closest to the origin, and the structure of the right-hand side of (1.7) clearly entails that a lower estimate of this distance - namely of the radius $\rho$ of $D$ - reads as follows:

$$
\rho>R \tilde{\zeta} / \tilde{\zeta}{ }^{\prime}
$$

with $R$ a positive constant (dependent on the values of the coupling constants $a_{n m}$ but not on the initial data) and $\tilde{\zeta}$ respectively $\tilde{\zeta}^{\prime}$ providing lower respectively upper estimates 
of the moduli of $\zeta_{n}(0)-\zeta_{m}(0)$ respectively $\zeta_{n}^{\prime}(0)$, say

$$
\begin{aligned}
& \tilde{\zeta}=\min _{n, m=1, \ldots, N ; n \neq m}\left|\zeta_{n}(0)-\zeta_{m}(0)\right|, \\
& \tilde{\zeta}^{\prime}=\max _{n=1, \ldots, N}\left|\zeta_{n}^{\prime}(0)\right|
\end{aligned}
$$

(for a derivation of this formula, including an explicit expression for $R$, see Appendix A). Let us now assume the initial data, see (1.8), to entail (via (1.9)) that $\rho>2 / \omega$. Then the disk $D$ includes the disk $C$, and this entails that the solutions $\underline{\zeta}(\tau)$ of (1.7) are holomorphic functions of the complex variable $\tau$ in the (closed) disk $C$, hence (see (1.6)) the corresponding solutions $\underline{z}(t)$ of $(1.5)$ are completely periodic functions of the real variable $t$, with period $T$, see (1.2).

This observation, together with the lower estimate (1.9) of $\rho$, imply the existence of a set, of nonvanishing (in fact, infinite) measure in phase space, of initial data $\underline{z}(0), \underline{\dot{z}}(0)$ which yield completely periodic solutions of (1.5) (hence as well of (1.1)). But before formulating this finding $[1,2]$ in the guise of the following Proposition [1.2 let us interject the following obvious

Remark 1.1. If $\underline{z}(t)$ is the solution of (1.5) corresponding to initial data $\underline{z}(0), \underline{\dot{z}}(0)$, then $\underline{\tilde{z}}(t)=c \underline{z}(b t)$ is the solution of the equations of motion that obtain from (1.5) by replacing in it $\omega$ with $\tilde{\omega}=b \omega$, and of course the corresponding initial data read $\underline{\tilde{z}}(0)=c \underline{z}(0)$, $\underline{\dot{\tilde{z}}}(0)=b c \underline{\dot{z}}(0)$. Here $b, c$ are of course arbitrary (nonvanishing!) rescaling constants.

Let us now formulate Proposition 1.2 (correcting thereby the partially incorrect formulation of this result given in Refs. [1,2]).

Proposition 1.2. Let $\underline{z}(t)$ be the solution of (1.5) with

$$
\omega=b \bar{\omega}
$$

corresponding to the assigned initial data

$$
\underline{z}(0)=c \underline{u}, \quad \underline{\dot{z}}(0)=\mu \underline{v}, \quad\left[\text { with } u_{n} \neq u_{m} \quad \text { if } \quad n \neq m\right],
$$

where the positive numbers $b, c, \mu$ play the role of scaling parameters (as we shall immediately see). Then the solution $\underline{z}(t)$ is completely periodic with period $T$, see (1.2),

$$
\underline{z}(t+T)=\underline{z}(t),
$$

provided one of the following conditions hold:

(i) for given $a_{n m}, \omega, \underline{z}(0)$ and $\underline{v}$, the scaling number $\mu$, hence as well the initial velocities $\dot{z}_{n}(0)$, are sufficiently small: $0 \leq \mu \leq \mu_{c}$, where $\mu_{c}$ is a positive number, $\mu_{c}>0$, whose value depends on the given quantities;

(ii) for given $a_{n m}, \omega, \underline{\dot{z}}(0)$ and $\underline{u}$, the scaling number $c$ is sufficiently large, $c>c_{c}$ (hence the initial positions of the $N$ particles in the plane are sufficiently well separated), with $c_{c}$ a positive number, $c_{c}>0$, whose value depends on the given quantities;

(iii) for given $a_{n m}, \bar{\omega}, \underline{z}(0)$ and $\underline{\dot{z}}(0)$, the scaling number $b$, hence as well the circular frequency $\omega=b \bar{\omega}$, is sufficiently large, $b>b_{c}$, where $b_{c}$ is a positive number, $b_{c}>0$, whose value depends on the given quantities. 
Remark 1.3. The first two formulations (items (i) and (ii)) of Proposition 1.2 refer to the same equations of motion, with modified (rescaled) initial conditions; the third formulation (item (iii)) refers to different equations of motion (due to the change via rescaling of the constant $\omega$ ) with the same initial conditions. But in fact these 3 formulations are completely equivalent, see Remark 1.1 .

We have thereby reviewed (and rectified!) the results of References [1,2], to an extent sufficient to make this paper self-contained. But before focusing on the specific, novel, contributions of this paper three additional facts are now recalled. The first two are relevant to the case - to which attention will be hereafter restricted - in which the (possibly complex) coupling constants $a_{n m}$ depend symmetrically on their two indices:

$$
a_{n m}=a_{m n} .
$$

Then the equations of motion (1.5) are Hamiltonian, being derivable in the standard manner from the Hamiltonian

$$
H(\underline{z}, \underline{p})=\sum_{n=1}^{N}\left\{i \omega z_{n} / c+\exp \left(c p_{n}\right) \prod_{m=1 ; m \neq n}^{N}\left[z_{n}-z_{m}\right]^{-a_{n m}}\right\}
$$

(and let us recall that this entails that the "physical" equations of motion (1.1) are as well Hamiltonian [2]). Note the presence, in this expression of the Hamiltonian function $H(\underline{z}, \underline{p})$, of the arbitrary (nonvanishing) constant $c$, which does not feature in the equations of motion (1.5). Also note that $H(\underline{z}, \underline{p})$, in contrast to the equations of motion (1.5), is not quite invariant under translations $\left(z_{n} \rightarrow \tilde{z}_{n}=z_{n}+z_{0}\right)$, although the only effect of such a translation on $H(\underline{z}, \underline{p})$ is addition of a constant.

Moreover, as clearly implied by the equations of motion (1.5) with (1.12), the center of mass,

$$
Z(t)=N^{-1} \sum_{n=1}^{N} z_{n}(t)
$$

moves periodically (with period $T$, see (1.2)) on a circular trajectory (in the complex $z$-plane):

$$
Z(t)=Z(0)+\dot{Z}(0)[\exp (i \omega t)-1] /(i \omega) .
$$

The third and last fact we like to recall is that, if all the coupling constants in (1.5) are unity,

$$
a_{n m}=1,
$$

then the equations of motion (1.5) are integrable indeed solvable [5, 2], the solution of the corresponding initial-value problem being given by the following neat prescription: the $N$ coordinates $z_{n}(t)$ which constitute the solution $\underline{z}(t)$ of the equations of motion (1.5) corresponding to the initial data $\underline{z}(0), \underline{\dot{z}}(0)$ are the $N$ roots of the following algebraic equation in the variable $z$ :

$$
\sum_{m=1}^{N} \dot{z}_{m}(0) /\left[z-z_{m}(0)\right]=i \omega /[\exp (i \omega t)-1]
$$


Note that, after elimination of all denominators, this is a polynomial equation of degree $N$ for the variable $z$, with all coefficients of the polynomial periodic in $t$ with period $T$, see (1.2). Hence the set $\underline{z}(t)$ of its $N$ zeros is as well periodic with period $T$. It is therefore clear that, in this special solvable case, see (1.16), all nonsingular solutions of (1.5) are completely periodic, with a period that is either $T$ or an integer multiple of $T$ (the latter possibility arises because the zeros of the polynomial - which is itself periodic with period $T$ - may get reshuffled through the motion - but of course that integer multiple cannot exceed $N$ ! - indeed it cannot exceed the combinatorial factor $p(N)$ defined as the smallest integer $p(N)$ such that the iteration $p(N)$ times of any permutation of $N$ objects yield unity, and of course $p(N) \ll N$ ! for large $N)$.

As we just saw, Proposition 1.2 entails that the equations of motion (1.5) (as well as the equivalent "physical" equations of motion (1.1)) possess a lot of nonsingular and completely periodic solutions. This finding [1, 2] was (of course!) validated by simulations performed via a computer program created by one of us (MS) to solve numerically the equations of motion (1.5). The new findings discussed in this paper emerged from the (rather successful!) effort to understand certain remarkable features of these numerical simulations - in particular the existence, in the cases characterized by real and rational values of the coupling constants $a_{n m}$ in (1.5), of additional sets of initial data, also of nonvanishing measure in phase space, yielding nonsingular and completely periodic solutions with periods which are integer multiples of $T$, see (1.2) - via a mechanism, at which we already hinted above, associated with rational branch points of the solutions $\zeta(\tau)$, which is indeed also responsible in the solvable case (with $a_{n m}=1$ ) to yield completely periodic solutions with such larger periods (integer multiples of $T$ ). These new findings also suggest the existence of other integrable - but presumably not solvable - cases of the many-body problem (1.1), as well as of nonintegrable cases for which however integrable behaviors, such as those associated with completely periodic motions, emerge from sectors of initial data having nonvanishing measure in phase space; and they display as well a mechanism for the emergence of a chaotic behavior (for other regions of phase space, of course only in the nonintegrable cases) characterized by a sensitive dependence on the initial data (the signature of chaos!) which is not associated with any local exponential divergence of trajectories over time - in analogy to the type of chaotic behavior that ensues when a particle moves freely (except for the reflections on the borders) inside, say, a triangular plane billiard whose angles are irrational fractions of $\pi$.

These findings shall be reviewed at the end of this paper, after they have been discussed in the body of it. In particular, in the following Section 2 we discuss the conservation laws associated with the equations of motion (1.7); in Section 3 we exhibit certain similarity solutions of these equations of motion; in Section 4 we discuss the solution of the two-body problem, in somewhat more detail than it was done in [1]; in Section 5 we investigate the analytic structure of the solutions of (1.7), in particular the nature of the branch points, in the complex $\tau$-plane, featured by the solutions of these equations of motion, and the structure of the associated Riemann surfaces, which is crucial to determine the behavior - be it completely periodic or chaotic - of the solutions of the "physical" equations of motion (1.5) namely (1.1) as the real time $t$ unfolds. The relevance of these findings for the motions of the "physical" problem (1.5) or (1.1) are then discussed, and also illustrated via the display of numerical simulations, in Section 6. But - to avoid this paper becoming excessively long - the treatments of Sections 5 and 6 are restricted to the case in which 
the real parts of the coupling constants $a_{n m}$ are nonnegative,

$$
\operatorname{Re}\left(a_{n m}\right) \geq 0,
$$

a condition which is sufficient (albeit not necessary; see below, in particular (5.8)) to exclude the phenomenon of "escape to infinity"; the general case shall be treated in a subsequent paper. A final Section 7 outlines how the study of this model can and should be pursued. To avoid interrupting the flow of the discourse certain technical developments are relegated to four Appendices.

\section{Conserved quantities}

The equations of motion (1.7) are of course (see (1.13)) implied by the Hamiltonian

$$
H(\underline{\zeta}, \underline{\pi})=\sum_{n=1}^{N}\left\{\exp \left(c \pi_{n}\right) \prod_{m=1 ; m \neq n}^{N}\left[\zeta_{n}-\zeta_{m}\right]^{-a_{n m}}\right\} .
$$

Indeed this yields the Hamiltonian equations of motion

$$
\begin{aligned}
& \zeta_{n}^{\prime}=\partial H / \partial \pi_{n}=c \exp \left(c \pi_{n}\right) \prod_{m=1 ; m \neq n}^{N}\left[\zeta_{n}-\zeta_{m}\right]^{-a_{n m}}, \\
& \pi_{n}^{\prime}=-\partial H / \partial \zeta_{n}=c^{-1} \sum_{m=1 ; m \neq n}^{N}\left(\zeta_{n}^{\prime}+\zeta_{m}^{\prime}\right)\left(\zeta_{n}-\zeta_{m}\right),
\end{aligned}
$$

where to write in more convenient form the second set of equations, (2.2b), we used the first set, (2.2a) (and we of course assumed validity of the symmetry property (1.12)). Clearly logarithmic differentiation of (2.2a) yields

$$
\zeta_{n}^{\prime \prime} / \zeta_{n}^{\prime}=c \pi_{n}^{\prime}-\sum_{m=1 ; m \neq n}^{N} a_{n m}\left(\zeta_{n}^{\prime}-\zeta_{m}^{\prime}\right) /\left(\zeta_{n}-\zeta_{m}\right),
$$

and these equations, via (2.2b), clearly yields the equations of motion (1.7).

It is thus seen, from (2.1) and (2.2a), that

$$
H=c^{-1} \sum_{n=1}^{N} \zeta_{n}^{\prime}
$$

hence in this case the fact that the Hamiltonian is a constant of the motion coincides with the time-independence of the "center-of-mass" velocity,

$$
(d / d \tau) \sum_{n=1}^{N} \zeta_{n}^{\prime}=0
$$

which is itself an immediate consequence of the equations of motion (1.7) (indeed, their sum over the index $n$ from 1 to $N$ implies the vanishing of the right-hand side due to 
the antisymmetry under exchange of the two dummy indices $n, m$ of the summand in the double sum - since we always assume (1.12) to hold).

A second conserved quantity, associated with the translation-invariant character of the Hamiltonian (2.1), is the total momentum $\Pi$,

$$
\Pi=\sum_{n=1}^{N} \pi_{n},
$$

as it is clear by summing over the index $n$ from 1 to $N$ the equations of motion (2.2b).

By exponentiation and via (2.2a) this entails the following version of the second conserved quantity:

$$
K=\prod_{n=1}^{N}\left[\zeta_{n}^{\prime} \prod_{m=1 ; m \neq n}^{N}\left(\zeta_{n}-\zeta_{m}\right)^{a_{n m}}\right]
$$

The fact that this quantity, $K$, is a constant of motion can also be verified as follows. Logarithmic differentiation of (2.7) yields

$$
\begin{aligned}
& K^{\prime} / K=\sum_{n=1}^{N}\left[\zeta_{n}^{\prime \prime} / \zeta_{n}^{\prime}+\sum_{m=1 ; m \neq n}^{N} a_{n m}\left(\zeta_{n}^{\prime}-\zeta_{m}^{\prime}\right) /\left(\zeta_{n}-\zeta_{m}\right)\right], \\
& K^{\prime} / K=\sum_{n=1}^{N}\left[\zeta_{n}^{\prime \prime} / \zeta_{n}^{\prime}-2 \sum_{m=1 ; m \neq n}^{N} a_{n m} \zeta_{m}^{\prime} /\left(\zeta_{n}-\zeta_{m}\right)\right]
\end{aligned}
$$

The second of these equations, (2.8b), follows from the first, (2.8a), by exchanging the role of the two dummy indices $n$ and $m$ in the first term of the double sum (and of course by taking advantage of (1.12)). It is then obvious, see (1.7), that $K^{\prime}$ vanishes, namely that $K$ is a constant of motion.

\section{Similarity solutions}

In this section we report certain special solutions of the evolution equations (1.7), hence as well, via (1.6), of the Newtonian equations of motion (1.5) and (1.1). They read (obviously, up to arbitrary permutations)

$$
\begin{aligned}
& \zeta_{n}(\tau)=c_{0}+c_{n}\left(\tau-\tau_{b}\right)^{\Gamma}, \quad n=1,2, \ldots, M, \\
& \zeta_{n}(\tau)=C_{n}, \quad n=M+1, M+2, \ldots, N,
\end{aligned}
$$

with $M$ a positive integer, $2 \leq M \leq N$, the complex constant $\tau_{b}$ arbitrary (it identifies the value of $\tau$ at which this solution has, generally, a branch point; see (3.1a) and below), the constants $C_{n}$ arbitrary (and largely irrelevant: particles that do not move, see (3.1b), can be altogether ignored, since the structure of the right-hand side of (1.7) entails that they neither feel nor cause any force), the constant $c_{0}$ also arbitrary (reflecting the translationinvariant character of the model), while the constants $\Gamma$ and $c_{n}$ are determined (the latter 
up to an arbitrary common multiplicative factor) by the following equations, which clearly obtain by inserting (3.1) in (1.7):

$$
\begin{aligned}
& \Gamma(\Gamma-1)=-\Gamma^{2} A, \\
& A=-2 \sum_{m=1 ; m \neq n}^{M} a_{n m} c_{m} /\left(c_{n}-c_{m}\right), \quad n=1,2, \ldots, M .
\end{aligned}
$$

The first of these equations, (3.2a), entails

$$
\Gamma=(1+A)^{-1}
$$

(provided $A \neq-1$; if instead $A=-1$ the function $\left(\tau-\tau_{b}\right)^{\Gamma}$ in the right-hand side of (3.1a) must be replaced by $\exp (\beta \tau)$, with $\beta$ an arbitrary nonvanishing constant; see below).

The $M$ equations (3.3a) determine $A$ (note the assumed independence of this quantity from the index $n$ ) and, up to a common rescaling factor, the $M$ constants $c_{m}$ with $m=$ $1,2, \ldots, M$. The expressions of these $M$ constants cannot be generally obtained (for arbitrary $a_{n m}$ ) in explicit form from (3.3a), but this is instead possible for $A$, hence, via (3.2b) for $\Gamma$ (provided, as we always assume, the symmetry property (1.12) of the coupling constants $a_{n m}$ hold). Indeed by replacing $c_{m}$ in the numerator in the right-hand side of (3.3a) with $\left(c_{m}-c_{n}\right)+c_{n}$ we get

$$
A=2 \sum_{m=1 ; m \neq n}^{M} a_{n m}-2 \sum_{m=1 ; m \neq n}^{M} a_{n m} c_{n} /\left(c_{n}-c_{m}\right),
$$

hence, by summing over $n$ from 1 to $M$,

$$
M A=2 \sum_{m, n=1 ; m \neq n}^{M} a_{n m}-M A .
$$

To obtain this equation, (3.3c), we exchanged the order of the second double sum over $n$ and $m$ in the right-hand side, we took advantage of the symmetry property (1.12), and we used again (3.3a). From (3.3C) (and taking again advantage of the symmetry property (1.12) so as to avoid any double counting in the sum) we finally get

$$
A=(2 / M) \sum_{n, m=1 ; n>m}^{M} a_{n m} .
$$

In conclusion we see that the system (1.7), for any arbitrary choice of the coupling constants $a_{n m}$, possesses the (exact) solution (3.1), with an arbitrary choice of the positive integer $M$ in the range $2 \leq M \leq N$ and of the $N-M$ (complex) constants $C_{m}$ (for $m$ in the range $M+1 \leq m \leq N)$, and with the $M$ constants $c_{n}$ (for $n$ in the range $1 \leq$ $n \leq M)$ determined, up to a common rescaling factor, by the system of $M$ algebraic equations (3.3a), where the constant $A$ is given (provided, as we always assume, there holds the symmetry property (1.12)) by the simple formula (3.3d). The (generally complex) 
constant $\tau_{b}$ in (3.1a) is also an arbitrary constant, and its significance is that, at $\tau=\tau_{b}$, the $M$ particles labeled from 1 to $M$ all "collide" if $\operatorname{Re}(\Gamma)>0$ or "escape to infinity" if $\operatorname{Re}(\Gamma)<0$, yielding of course a singularity of the equations of motion, as evidenced by the right-hand side of (1.7). Note that the solutions $\zeta_{n}(\tau)$ possess, at this point $\tau=\tau_{b}$, a branch point singularity, whose nature is characterized by the exponent $\Gamma$, see (3.1a) and (3.2b) with (3.3d). Of course analogous solutions exist for any arbitrary permutation of the $N$ indices $n$. (Note that above we put inverted commas around the word "collide" or "escape to infinity" - to underline that this is not, generally, a true "physical" phenomenon, since it occurs at some complex value of the $\tau$ variable; see below).

The corresponding solutions $\underline{z}(t)$ of the equations of motion (1.5), hence as well (via (1.4)) of the Newtonian equations of motion (1.1), obtain from these solutions $\underline{\zeta}(\tau)$ via (1.6). Therefore when the complex constant $\tau_{b}$ falls outside the disk $C$ (centered, in the complex $\tau$-plane, at $\tau=i / \omega$, and with radius $1 / \omega)$, these solutions $\underline{z}(t)$ are nonsingular and completely periodic with period $T$, see (1.2), while if the complex point $\tau_{b}$ falls inside the disk $C$, the solutions $\underline{z}(t)$ are again nonsingular but generally not periodic, unless the exponent $\Gamma$, see (3.1a ) and (3.2b) with (3.3d) is rational, $\Gamma=p / q$ (with $p$ and $q$ two coprime integers, and, say, $q$ positive, $q \geq 1$ ), in which case the solutions $\underline{z}(t)$ are again completely periodic, but with period $\tilde{T}=q T$ (as clearly entailed by (3.1a) with (1.6)). In the special case in which the point $\tau_{b}$ falls exactly on the circle $\tilde{C}$ that constitutes the boundary of the disk $C$, so that the formula $\tau_{b}=\left[\exp \left(i \omega t_{c}\right)-1\right] /(i \omega)$ defines $\bmod (T)$ a real time $t_{c}$, then the solution $\underline{z}(t)$ corresponds to a special Newtonian motion in which, at the "collision time" $t_{c}, M$ particles simultaneously collide (if $\operatorname{Re}(\Gamma)>0$ ) or escape to infinity (if $\operatorname{Re}(\Gamma)<0$ ).

This analysis applies provided $A \neq-1$ (see (3.3d ) ), so that $\Gamma$ is well defined (see $(3.2 \mathrm{~b})$ ). If instead $A=-1$, the similarity solutions $\underline{\zeta}(\tau)$ are entire functions of $\tau$, since the expression (3.1a) is then replaced by the formula

$$
\zeta_{n}(\tau)=c_{n} \exp (\beta \tau),
$$

where $\beta$ is an arbitrary constant (just as $\tau_{b}$ is an arbitrary constant in (3.1a)). Hence in this case the corresponding similarity solutions $\underline{z}(t)$ are always nonsingular and completely periodic with period $T$, see (1.2), as indeed clearly entailed by (3.4) with (1.5).

The actual behavior of these similarity solutions $\underline{z}(t)$ need not be discussed any further, nor explicitly displayed, since the interested reader will have no difficulty to figure it out from (3.1a) (or (3.4) ) and (1.6). Let us only note that, in the "equal particle" case in which all the coupling constants $a_{n m}$ coincide,

$$
a_{n m}=a,
$$

the expression (3.3d) yields

$$
A=a(M-1),
$$

hence (3.2b yields (provided $A \neq-1$; otherwise, see the previous paragraph)

$$
\Gamma=[1+a(M-1)]^{-1},
$$

and in this special case the constants $c_{n}$ can be explicitly exhibited,

$$
c_{n}=c \exp (2 i \pi n / M) \text {, }
$$


consistently, see (3.3a) and (3.5b), with the identities

$$
\begin{gathered}
\sum_{m=1 ; m \neq n}^{N} \exp (2 i \pi m / M) /[\exp (2 i \pi n / M)-\exp (2 i \pi m / M)]=-(M-1) / 2, \\
n=1,2, \ldots, M .
\end{gathered}
$$

The treatment of this special "equal particle" case is not new $[4,2]$.

Let us finally note that, even though in general the constants $c_{m}$ cannot be determined in explicit closed form in the general case with arbitrary $N$ and different coupling constants $a_{n m}$, whenever these depend symmetrically from their two indices, see (1.12), there holds the sum rule

$$
\sum_{n=1}^{N} c_{n}=0,
$$

which clearly obtains by multiplying (3.3a) by $c_{n}$ and summing over the index $n$ from 1 to $N$ (since the double sum in the right-hand side then vanishes due to the antisymmetry of the summand). For $N=2$ this sum rule is sufficient to determine the constants $c_{n}$ (up to a common rescaling constants), since it clearly entails $c_{1}=c, c_{2}=-c$.

\section{The two-body problem}

In this section we provide a somewhat more complete treatment of the two-body problem than given in [1]. This is of interest in itself, but even more so for the insight it provides, not only for the two-body case but as well for the $N$-body case (as discussed in the following Section 5), on the nature of the singularities of the solutions $\underline{\zeta}(t)$ of $(1.7)$ as functions of the complex variable $\tau$, hence on the periodicity of the corresponding solutions $\underline{z}(t)$ of the "physical" equations of motion (1.5).

For $N=2$ the equations of motion (1.7) are consistent with the assignment (corresponding to the standard separation of the center of mass and relative motions)

$$
\begin{aligned}
& \zeta_{1}(\tau)+\zeta_{2}(\tau)=\zeta_{1}(0)+\zeta_{2}(0)+V \tau, \\
& \zeta_{1}(\tau)-\zeta_{2}(\tau)=\zeta(\tau)
\end{aligned}
$$

namely

$$
\begin{aligned}
& \zeta_{1}(\tau)=\left[\zeta_{1}(0)+\zeta_{2}(0)+V \tau+\zeta(\tau)\right] / 2, \\
& \zeta_{2}(\tau)=\left[\zeta_{1}(0)+\zeta_{2}(0)+V \tau-\zeta(\tau)\right] / 2,
\end{aligned}
$$

where (see (1.14), (1.8) and (4.1a))

$$
V=\zeta_{1}^{\prime}(0)+\zeta_{2}^{\prime}(0)=\zeta_{1}^{\prime}(\tau)+\zeta_{2}^{\prime}(\tau)=2 \dot{Z}(0)
$$

is a (generally complex) constant and the difference $\zeta(\tau)$ satisfies the second-order ODE

$$
\zeta^{\prime \prime}=a\left[V^{2}-\left(\zeta^{\prime}\right)^{2}\right] / \zeta
$$


Here and throughout this section $a=a_{12}=a_{21}$ is the relevant "coupling constant", and primes denote of course differentiations with respect to $\tau$.

This ODE is easily integrated once (after multiplying it by the factor $2 \zeta^{\prime} /\left[V^{2}-\left(\zeta^{\prime}\right)^{2}\right]$ ), and one gets thereby

$$
\begin{aligned}
& \left(\zeta^{\prime}\right)^{2}=V^{2}+B \zeta^{-2 a} \\
& \left(\zeta^{\prime}\right)^{2}=V^{2}\left[1+(\zeta / L)^{-2 a}\right],
\end{aligned}
$$

with $B=-4 \zeta_{1}^{\prime}(0) \zeta_{2}^{\prime}(0)[\zeta(0)]^{2 a}-$ or, when $V \neq 0$, equivalently but notationally more conveniently, $L=\left(B / V^{2}\right)^{1 /(2 a)}=\zeta(0)\left\{\left[\zeta^{\prime}(0) / V\right]^{2}-1\right\}^{1 /(2 a)}-$ a (generally complex) integration constant.

In the special case $V=0$ the first-order ODE (4.4a) can be easily integrated once more, and one obtains thereby $[1,2]$ the solution of (4.3) in closed form: for $a \neq-1$,

$$
\zeta(\tau)=c\left(\tau-\tau_{b}\right)^{\gamma}
$$

with

$$
\gamma=1 /(1+a)
$$

for $a=-1$,

$$
\zeta(\tau)=c \exp (\beta \tau)
$$

Here $c, \tau_{b}$ and $\beta$ are arbitrary (complex) constants, and it is easily seen that $\tau_{b}$ respectively $\beta$ are related to the initial data by the relations

$$
\tau_{b}=-(1+a)^{-a} \zeta(0) / \zeta^{\prime}(0)
$$

respectively

$$
\beta=\zeta^{\prime}(0) / \zeta(0)
$$

Note that this solution coincides with the similarity solution (3.1) (with $N=M=2$, $\gamma=\Gamma)$.

For $V \neq 0$ the ODE (4.4) can of course be generally integrated by a further quadrature, but the corresponding formula in terms of the hypergeometric function $F(A, B ; C ; Z)[6]$,

$$
\zeta F\left(1 / 2,-1 /(2 a) ; 1-1 /(2 a) ;[\zeta / L]^{-2 a}\right)=V\left(\tau-\tau_{b}\right),
$$

is not particularly enlightening, except in the special cases listed below in which the hypergeometric function reduces to elementary functions (actually in these cases direct integration of (4.4) is the neatest way to get the solution, without going through the hypergeometric function).

For $a=-1$, one easily finds

$$
\zeta(\tau)=L \sinh \left[(V / L)\left(\tau-\tau_{0}\right)\right],
$$


with $\tau_{0}$ a (complex) constant (related of course to the initial data: $\left.\zeta(0)=-L \sinh \left[(V / L) \tau_{0}\right]\right)$. Hence in this case $\zeta(\tau)$ is an entire function of $\tau$, and via (1.6) this entails that all solutions of the Newtonian equations of motion (1.5) (with $N=2$ ) are in this case nonsingular and completely periodic with period $T$, see (1.2) (as entailed by (4.1) with (4.7) or (4.9)).

For $a=1$ (namely, for the special value of the coupling constant that corresponds, in the equal-particle $N$-body problem, to the solvable case, see (1.17)), it is as well easy to get

$$
\zeta(\tau)=V\left[\left(\tau-\tau_{+}\right)\left(\tau-\tau_{-}\right)\right]^{1 / 2}
$$

with the two (complex) constants $\tau_{ \pm}$related to the initial values by the formula

$$
\tau_{ \pm}=\left[\zeta_{2}(0)-\zeta_{1}(0)\right]\left\{\left[\zeta_{1}^{\prime}(0)\right]^{1 / 2} \pm i\left[\zeta_{2}^{\prime}(0)\right]^{1 / 2}\right\}^{2} / V^{2}
$$

Note the square-root branch point in the right-hand side of 4.10a , and its consistency with (4.5) and (4.6) (of course with $a=1$ ). It is easily seen, via (1.6), that these findings entail the following results for the solutions $z_{n}(t)$ of the Newtonian equations of motion (1.5) (with $N=2$ and $a=1$ ). If $V=0$ (namely, if the center of mass does not move - initially, hence as well throughout the motion, see (1.15)), the solutions are nonsingular and completely periodic with period $T$, see (1.2), if (the initial data entail, see (4.5b) and (1.8), that) $\tau_{b}$ falls, in the complex $\tau$-plane, outside the circular disk $C$ (centered at $\tau=i / \omega$ and of radius $1 / \omega$ ); they are nonsingular and completely periodic with period $\tilde{T}=2 T$ if $\tau_{b}$ instead falls inside the circular disk $C$; while they are singular (due to the occurrence of a two-body collision) at the time $t_{c}$ defined $\bmod (T)$ by the formula $\tau_{b}=\left[\exp \left(i \omega t_{c}\right)-1\right] /(i \omega)$, if $\tau_{b}$ falls just on the boundary of $C$, namely on the circular contour $\tilde{C}$ (which is just the condition necessary and sufficient to entail that $t_{c}$, as defined above, be real). Likewise, if instead $V \neq 0$ (in which case the center of mass moves on a circular orbit, see (1.15)), the solutions are nonsingular and completely periodic with period $T$, see (1.2), if (the initial data entail, see (4.10b) and (1.8), that) the two complex constants $\tau_{ \pm}$fall either both inside or both outside the circular disk $C$; they are nonsingular and completely periodic with period $\tilde{T}=2 T$ if one of the two constants $\tau_{ \pm}$ falls inside the circular disk $C$ and the other outside it; while they are singular (due to the occurrence of a two-body collision) if $\tau_{+}$or $\tau_{-}$falls just on the boundary of $C$, namely on the circular contour $\tilde{C}$. These results of course confirm those entailed by the resolvent formula (1.17), as described after that formula, see above. (If one considers initial data of type (1.10b) with fixed $z_{n}(0)=\zeta_{n}(0)$ and $\dot{z}_{n}(0)=\zeta_{n}^{\prime}(0)=\mu v_{n}$ entailing $V=\mu\left(v_{1}+v_{2}\right)$ with fixed $v_{n}$, then (4.10b) yields $\tau_{ \pm} \equiv \tau_{ \pm}(\mu)=\mu^{-1} \tau_{ \pm}(1)$, hence the motion is completely periodic with period $T$, see (1.2), both for sufficiently small and for sufficiently large values of the positive scaling parameter $\mu$; it is instead completely periodic with period $\tilde{T}=2 T$ for a finite intermediate interval of values of $\mu$ ).

A third neatly solvable case obtains if $a=-1 / 2$. Then

$$
\zeta(\tau)=L\left\{\left[(V / L)\left(\tau-\tau_{0}\right) / 2\right]^{2}-1\right\},
$$

which clearly entails that $\zeta(\tau)$ is entire (indeed, just a second-degree polynomial) in $\tau$. Note that the same conclusion is implied in this case by (4.5a) with (4.6). Hence we conclude that in this case all solutions of the Newtonian equations of motion (1.5) (can 
be exhibited in explicit closed form and) are nonsingular and completely periodic with period $T$, see (1.2).

The next two cases the solutions of which we report explicitly are characterized by $a=-2$ and $a=-3$, respectively (the alert reader can easily check these results, or derive them via the approach of Appendix B).

For $a=-2$ the solution reads

$$
\zeta(\tau)=\lambda \zeta(0)\left\{\operatorname{cn}\left(\left\{V\left(\tau-\tau_{0}\right) /[\lambda \zeta(0)]\right\}, 2^{-1 / 2}\right)\right\}^{-1} .
$$

Here and below $\mathrm{cn}(u, k)$ is the standard Jacobian elliptic function (see for instance [6]), and the two constants $\lambda$ and $\tau_{0}$ are related to the initial data as follows:

$$
\begin{aligned}
& \lambda=2^{-1 / 4}\left[\zeta^{\prime}(0) / V\right]^{-1 / 2}, \\
& \operatorname{cn}\left(\left\{V \tau_{0} /[\lambda \zeta(0)]\right\}, 2^{-1 / 2}\right)=\lambda .
\end{aligned}
$$

These formulas entail that, in this case with $N=2$ and $a=a_{12}=a_{21}=-2$, all solutions $\zeta_{n}(\tau)$ of the equations of motion (1.7) are meromorphic functions of $\tau$ (note that this is also the case if $V=0$, because $a=-2$ entails $\gamma=-1$; see (4.5) and (4.6)). Hence, in this case with $N=2$ and $a=a_{12}=a_{21}=-2$, all nonsingular solutions $z_{n}(t)$ of the "physical" equations of motion (1.5) or (1.1) are completely periodic functions of the time $t$, with period $T$, see (1.2).

For $a=-3$ the solution reads

$$
\begin{aligned}
& \zeta(\tau)=\lambda \zeta(0)\left\{\wp\left(\left\{V\left(\tau-\tau_{0}\right) /[\lambda \zeta(0)]\right\} ; 0,-4\right)\right\}^{-1 / 2}, \\
& \zeta(\tau)=V\left[\wp\left(\left(\tau-\tau_{0}\right) ; 0,-4[V / \zeta(0)]^{6}\left\{-1+\left[\zeta^{\prime}(0) / V\right]^{2}\right\}\right)\right]^{-1 / 2} .
\end{aligned}
$$

Here and below $\wp\left(u ; g_{2}, g_{3}\right)$ is the standard Weierstrass elliptic function (see for instance [6]), and the two constants $\lambda$ and $\tau_{0}$ are related to the initial data as follows:

$$
\begin{aligned}
& \lambda=\left\{-1+\left[\zeta^{\prime}(0) / V\right]^{2}\right\}^{-1 / 6}, \\
& \lambda^{2} \wp\left(\left\{V \tau_{0} /[\lambda \zeta(0)]\right\} ; 0,-4\right)=1
\end{aligned}
$$

(the equivalence of (4.13a) and (4.13b) is entailed by the rescaling properties of the Weierstrass function, see for instance eq. 14.13(2) of [6]). These formulas entail that, in this case with $N=2$ and $a=a_{12}=a_{21}=-3$, the only singularities featured by the solutions $\zeta(\tau)$ of the equations of motion (1.7) as functions of $\tau$ are branch points with exponent $-1 / 2$ (note that this is also the case if $V=0$, because $a=-3$ entails $\gamma=-1 / 2$; see (4.5) and (4.6) ). Hence, in this case with $N=2$ and $a=a_{12}=a_{21}=-3$, all nonsingular solutions $\underline{z}(t)$ of the "physical" equations of motion (1.5) or (1.1) are completely periodic functions of the time $t$, with period $T$, see (1.2), or $\tilde{T}=2 T$.

The last case we treat in detail is characterized by $a=-1 / 4$. It can then be shown (again, via simple calculations analogous to those carried out in Appendix B) that in this case the general solution of $4.4 \mathrm{~b}$ ) (with $V \neq 0$ ) is given by the (implicit) formula

$$
[\zeta(\tau) / L]^{3 / 2}-3[\zeta(\tau) / L]=(9 / 16)\left[(V / L)\left(\tau-\tau_{b}\right)\right]^{2}-3(V / L)\left(\tau-\tau_{b}\right),
$$


entailing

$$
\zeta(\tau)=V\left(\tau-\tau_{b}\right) \sum_{l=0}^{\infty} \alpha_{l}\left[(V / L)\left(\tau-\tau_{b}\right)\right]^{l / 2}
$$

with

$$
\alpha_{0}=\alpha_{1}=1, \quad \alpha_{2}=-3 / 16, \quad \ldots
$$

It is thus seen that, in this case with $a=-1 / 4, \zeta(\tau)$ has a square-root branch point at $\tau=\tau_{b}$ if the center of mass moves $(V \neq 0)$, while it has a cubic-root branch point if the center of mass does not move $(V=0$; indeed $a=-1 / 4$ entails $\gamma=4 / 3$, see (4.5a) and (4.6)). Hence, in this case with $a=-1 / 4$, the solutions $z_{n}(t)$ of the physical twobody problem (1.5) are nonsingular and completely periodic with period $T$, see (1.2), if the branch point $\tau_{b}$ (whose location depends of course on the initial conditions, see 4.5b) or 4.14a) fall outside the circular disk $C$ (centered in the complex $\tau$-plane at $\tau=i / \omega$ and of radius $1 / \omega$ ); they are of course singular at a finite real time $t=t_{c}$ (when the two particles collide) if $\tau_{b}$ falls just on the boundary of $C$; while if the branch point $\tau_{b}$ falls inside the circular disk $C$ they are nonsingular and completely periodic with period $\tilde{T}=2 T$ or $\tilde{T}=3 T$ depending whether the center of mass does $(V \neq 0)$ or does not $(V=0)$ move.

As implied by our general treatment and exemplified by these examples, an analogous analysis of the emergence of periodic motions in the two-body problem (1.5) can also be made for an arbitrary value of the coupling constant $a$. The conclusions of such an analysis depend essentially on the nature, and location, of the branch points featured by the solutions of the first-order ODE (4.4) (with $V \neq 0$; the $V=0$ case is completely illuminated by the explicit solution (4.5) with (4.6), hence our treatment below refers exclusively to the $V \neq 0$ case). A detailed treatment of this question is given in Appendix B, and it leads to the following results.

Three cases must be distinguished, depending on the value of the real part of the coupling constant $a$.

Case (i):

$$
\operatorname{Re}(a)>0 \text {. }
$$

In this case the branch point at, say, $\tau=\tau_{b}$, is characterized by the exponents $\gamma$ and $1-\gamma$, see (4.6), with the behavior of $\zeta(\tau)$ for $\tau \approx \tau_{b}$ given by the formula

$$
\begin{aligned}
\zeta(\tau)= & L \gamma^{-\gamma}\left[(V / L)\left(\tau-\tau_{b}\right)\right]^{\gamma} \\
& \times\left\{1+\sum_{l=1}^{\infty} \sum_{k=0}^{l} g_{k l}\left[(V / L)\left(\tau-\tau_{b}\right)\right]^{k \gamma}\left[(V / L)\left(\tau-\tau_{b}\right)\right]^{2 l(1-\gamma)}\right\}
\end{aligned}
$$

(for a justification of this formula, including the significance of the coefficients $g_{k l}$, see (B.20) with (B.2)). Note that this formula entails $\zeta\left(\tau_{b}\right)=0$ (as well as $\left|\zeta^{\prime}\left(\tau_{b}\right)\right|=\infty$, since (4.6) and (4.15a) entail $0<\operatorname{Re}(\gamma)<1$ ), namely this singularity is associated with a "collision" of the two particles (see (4.1b) ), which both move with infinite speed when they collide. This singularity is analogous to that which obtains in the $V=0$ case, see (4.5a). 
Case (ii):

$$
-1<\operatorname{Re}(a)<0 .
$$

In this case the branch point at, say, $\tau=\tau_{b}$, is characterized by the exponent

$$
\beta=-2 a,
$$

with the behavior of $\zeta(\tau)$ for $\tau \approx \tau_{b}$ given by the formula

$$
\zeta(\tau)= \pm V\left(\tau-\tau_{b}\right)\left\{1+\sum_{l=1}^{\infty} g_{l}\left[ \pm(V / L)\left(\tau-\tau_{b}\right)\right]^{l \beta}\right\} .
$$

This singularity, however, is generally not of the same type as that which obtains in the $V=0$ case, see (4.5a) (except for those values of $a$ such that $\gamma=(1+a)^{-1}$ and $\beta=-2 a$ differ by an integer). In both cases ( $V=0$, see (4.5a) with (4.6) and 4.16a); $V \neq 0$, see (4.16)) $\zeta\left(\tau_{b}\right)=0$, namely this singularity is again associated with a "collision" of the two particles (see (4.1b) ); however, in the $V=0$ case $\left|\zeta^{\prime}\left(\tau_{b}\right)\right|=\infty$, while in the $V \neq 0$ case $\zeta^{\prime}\left(\tau_{b}\right)= \pm V$ and this entails (see (4.1)) that either $\zeta_{1}^{\prime}(\tau)$ or $\zeta_{2}^{\prime}(\tau)$ vanishes at $\tau=\tau_{b}$ so that

$$
\zeta_{1}^{\prime}\left(\tau_{b}\right) \zeta_{2}^{\prime}\left(\tau_{b}\right)=0
$$

Case (iii):

$$
\operatorname{Re}(a)<-1
$$

In this case both behaviors, (4.15b) respectively (4.16c), are possible, so both type of branch points, characterized by the exponents $\gamma=(1+a)^{-1}$ respectively $\beta=-2 a$, may be present: but the first type of branch point, characterized by the exponent $\gamma$ (which obtains now for all values of $V$ ) corresponds now to the phenomenon of "escape to infinity" $\left(\left|\zeta\left(\tau_{b}\right)\right|=\left|\zeta^{\prime}\left(\tau_{b}\right)\right|=\infty\right)$, while the second type of branch point, characterized by the exponent $\beta$ (which obtains only if $V$ does not vanish, $V \neq 0$ ) corresponds to the phenomenon of "two-body collision" $\left(\zeta\left(\tau_{b}\right)=0\right.$, with $\zeta^{\prime}\left(\tau_{b}\right)= \pm V$ entailing (4.16d) $)$.

The behaviors of the corresponding solutions, $\underline{z}(t)$, of the "physical" equations of motion (1.5) depends on the locations of the branch points $\tau_{b}$ in the complex $\tau$-plane, which of course depend themselves on the initial data (and on the coupling constant $a$ ). (Note that the above analysis is local, namely it applies in the neighborhood of each branch point; nothing excludes that there be several, or possibly an infinity, of them). If none of these branch points is located inside the disk $C$ (centered in the complex $\tau$-plane at $\tau=i / \omega$ and of radius $1 / \omega)$ nor on its boundary $\tilde{C}$, then the solutions $z_{n}(t)$ are nonsingular and completely periodic with period $T$, see (1.2) (irrespective of the value of the coupling constant $a$ ). If instead one branch point, say at $\tau=\tau_{b}$, falls inside the disk $C$, then the motion is again nonsingular but generally not periodic, unless the branch point exponent is (real and) rational, in which case the motion may again be nonsingular and completely periodic (see below for a justification of the conditional), but with a larger period $\tilde{T}=q T$, where $q$ is the (positive) integer in the denominator of the rational exponent characterizing the branch point, which depends of course on the two-body "coupling 
constant", as discussed above (indeed a necessary and sufficient condition for the branch point exponent to be rational is that the coupling constant $a$ be itself rational - except for the special case $a=-1$, when the motion, see (4.7) and (4.9), is always nonsingular and completely periodic with period $T$, see (1.2)). And if $\tau_{b}$ falls just on the boundary of $C$, namely on the circular contour $\tilde{C}$, then at a finite real time $t_{c}$ defined $\bmod (T)$ by the formula $\tau_{b}=\left[\exp \left(i \omega t_{c}\right)-1\right] /(i \omega)$ the "physical" equations of motion (1.5) become generally singular, either because the two particles collide, or because they escape to infinity (as the case may be, see above). Note however that, if $a$ is a negative integer or a negative half-integer, in the case in which the two particles collide there is no singularity; this is entailed by (4.16c), it is consistent with the occurrence of a collision thanks to (4.16d), and it is indeed verified in the special cases $a=-2, a=-3, a=-1 / 2$, see (4.12), (4.13), (4.14). If more than one branch point occurs inside the disk $C$ the analysis must be adjusted accordingly; of course the outcome is critically affected not only by the presence of such branch points, but as well by which sheets they are located on, namely it depends on the overall structure of the Riemann surface associated with $\underline{\zeta}(\tau)$, the key element being always the path traveled on that surface by the complex point $\tau=[\exp (i \omega t)-1] /(i \omega)$ as the real variable $t$ ("time") evolves onward from the initial moment $t=0$.

In conclusion let us emphasize that our findings identify the following reasoning as instrumental to understand the motion of the particles in the plane entailed, for given initial data, by the Newtonian equations of motion (1.1), or equivalently by the complex equations of motion (1.5). The idea is to fix attention on the solution of (1.7) (rather than (1.5)) corresponding to the same initial data (see (1.8)). This defines the solution $\zeta_{n}(\tau)$ (with $n=1,2$, since we are now restricting attention to the two-body case; but we will see in the next section that essentially the same reasoning applies in the $N$-body case), to which is generally associated a multi-sheeted Riemann surface in the complex $\tau$-plane. The behavior of the solution of the "physical" equations of motion (1.5) as a function of the, of course real, time $t$ is then obtained by traveling on that Riemann surface following the circular contour $\tilde{C}$ defined by (1.6). Depending on the structure of the Riemann surface, this may entail a motion that is nonsingular and completely periodic (with period $T$, see (1.2), or with a period which is an integer multiple of $T$ ), that is singular (if a branch point happens to sit just on the contour $\tilde{C}$ ), or that is nonsingular but not periodic. Two mechanisms may give rise to the latter outcome (no periodicity): (i) the nature of the branch points, if they are characterized by an exponent that is not a real rational number (whether this is going or not to happen is immediately predictable, since it depends on whether the coupling constant $a$ is or is not itself a real rational number, see (4.6) and (4.16b) ); (ii) even if the coupling constant is a real rational number, so that each branch point yields only a finite number of sheets, there still may be an infinite number of sheets due to an infinity of branch points (of course not all of them occurring necessarily on the same sheet, but possibly in a nested fashion), and it may then happen that by traveling along the contour $\tilde{C}$ an endless sequence of new sheets is accessed (a necessary condition for this to happen is that the Riemann surface feature an infinity of branch points inside the contour $\tilde{C}$ - of course, on different sheets). Of course both mechanisms could be at work simultaneously.

Clearly the completely periodic motions correspond to an integrable behavior of the system; and, as shown above, whatever the value of the coupling constant $a$, there always exist at least a set (having nonvanishing, indeed infinite, measure in phase space) 
of initial data which yield such a behavior (nonsingular and completely periodic motions with period $T$, see (1.2)). As for the motions which are instead not periodic, one may well ask whether they should be categorized as chaotic or as not chaotic. It seems natural to suggest that, if the chaotic behavior is characterized by the fact that two trajectories, however close the initial data are that define them, give eventually rise to completely different motions ("sensitive dependence on initial conditions"), then clearly the nonperiodic motions will be chaotic respectively not chaotic according to whether it is infinite respectively finite the number of branch points which, by falling just inside or just outside the circular contour $\tilde{C}$, determine the (infinite) number of sheets that are accessed by a path following that contour $\tilde{C}$ on the Riemann surface associated with the solution $\underline{\zeta}(\tau)$ of (1.7). Indeed, if that number of branch point is finite, two sets of initial data that are sufficiently close to each other (in phase space) yield two Riemann surfaces which are sufficiently similar to each other so that, throughout the two motions corresponding to the two sets of initial data, the same sequence of sheets is traveled. But if that number of branch points is infinite, then even two solutions $\underline{\zeta}(\tau)$ of $(1.7)$ that are initially very close will inevitably be associated with two Riemann surfaces in which one relevant branch points falls in one case inside, and in the other outside, the circular contour $\tilde{C}$, hence, by traveling on $\tilde{C}$, after that point has been passed the corresponding trajectories of the physical problem (1.5) (or, equivalently, (1.1)) become different, because from that moment a different sequence of sheets is accessed of the two Riemann surfaces associated with the corresponding solutions $\underline{\zeta}(\tau)$ of the evolution equations (1.7). This is then to be interpreted as the cause of chaos. Note however that, even in the chaotic case, there is no local exponential divergence of trajectories; the mechanism that causes the onset of chaos in this case is rather analogous to that which characterizes the nonperiodic free motion of a point in, say, a triangular plane billiard with angles which are irrational fractions of $\pi$ (then any two trajectories, however close they initially are - and for however long they remain close - eventually become topologically different because one of the two misses a reflection that the other one takes, and from that moment onwards their evolutions become quite different).

\section{Branch points of the solutions of (1.7) in the $N$-body case}

In this Section we investigate the branch point structure in the complex $\tau$-plane of the solutions $\underline{\zeta}(\tau)$ of the equations of motion (1.7), since - as discussed above - the nature and location of these branch points determine the behavior of the solutions $\underline{z}(t)$ of the equations of motion (1.5), namely of the Newtonian equations of motion (1.1), as functions of the real time variable $t$. Let us re-emphasize that it is indeed the structure of the Riemann surface associated with the solution $\underline{\zeta}(\tau)$ of the equations of motion (1.7) that determines whether the corresponding solution $\underline{z}(t)$ of the "physical" equations of motion (1.5) namely (1.1) does or does not become singular as function of the real time variable $t$, and if it is not singular throughout time whether or not it is completely periodic, and if it is periodic then with what period (whether $T$, see (1.2), or an integer multiple of $T$ ). The rule to evince these conclusions is quite simple, see (1.6): to obtain $\underline{z}(t)$ as function of the real variable $t$ one must follow the corresponding solution $\underline{\zeta}(\tau)$ (namely, that characterized by the same initial data, see (1.8)) as the complex "time-like" variable $\tau$ travels, on the 
Riemann surface associated with that solution $\underline{\zeta}(\tau)$, around and around counterclockwise along the circular contour $\tilde{C}$ centered at $i / \omega$ and of radius $1 / \omega$.

The implications of this analysis have already been discussed in the preceding Section 4 in the context of the two-body problem. The situation in the $N$-body case is somewhat analogous. Indeed the structure of the evolution equations (1.7) entails that the same two mechanisms discussed in the two-body case are generically responsible for the emergence of singularities, at some complex values $\tau_{b}$, of the solutions of these equations of motion, (1.7), for an arbitrary number $N$ of particles: namely, singularities arise either from the "collision" of two particles, characterized by the relation $\zeta_{1}\left(\tau_{b}\right)=\zeta_{2}\left(\tau_{b}\right)$ (with $\left|\zeta_{1}\left(\tau_{b}\right)\right|=$ $\left|\zeta_{2}\left(\tau_{b}\right)\right|<\infty$, where we assign, without loss of generality, the labels 1 and 2 to the two "colliding" particles), or from the simultaneous "escape to infinity" of two or more (say, $M$ ) particles, characterized by the relation $\left|\zeta_{1}\left(\tau_{b}\right)\right|=\left|\zeta_{2}\left(\tau_{b}\right)\right|=\cdots=\left|\zeta_{M}\left(\tau_{b}\right)\right|=\infty$ (where, without loss of generality, we assumed the $M$ "particles" $\zeta_{n}(\tau)$ that escape to infinity as $\tau \rightarrow \tau_{b}$ to be labeled by the first $M$ indices, $\left.n=1, \ldots, M\right)$. Note that here we use again inverted commas around the word "collision" and "escape to infinity" to underline that only in the special cases in which, via the transformation (1.6), to the value $\tau_{b}$ there corresponds a real value $t_{c}$ (namely, $\tau_{b}=\left[\exp \left(i \omega t_{c}\right)-1\right] /(i \omega)$ with $t_{c}$ real; see $(1.6)$ ), the "collision", or the "escape to infinity", corresponds indeed to a real event for the physical problem (1.5) namely (1.1).

The statement we just made is not meant to exclude the possibility that "collisions" involving simultaneously more than two particles occur and cause correspondingly a singularity: indeed the exact similarity solutions (with $M>2$ ) presented in Section 3 provide examples of solutions characterized by such phenomena. But it stands to reason - and it is confirmed by our analysis, see below - that $M$-particle "collisions" with $M>2$ are not generic, namely they are not associated to solutions emerging from the assignment of generic initial data: indeed for a generic solution of the equations of motion (1.7) the complex equation $\zeta_{1}\left(\tau_{b}\right)=\zeta_{2}\left(\tau_{b}\right)$ generally has at least one complex solution $\tau_{b}$ (and more likely many such solutions, indeed quite possibly an infinity of them), while one should not expect the $M-1$ complex equations $\zeta_{1}\left(\tau_{b}\right)=\zeta_{2}\left(\tau_{b}\right)=\cdots=\zeta_{M}\left(\tau_{b}\right)$, with $M>2$, to possess any solution at all (although there are of course special solutions $\underline{\zeta}(\tau)$ of $(1.7)$ for which such multiple equations do possess solutions, see for instance Section 3). Let us then understand the type of singularity associated to these two types of "events". To this end the discussion of the preceding Section 4 is helpful (especially to guess at the nature of the singularity associated with such "events"), but a new treatment in the $N$-body context is nevertheless necessary.

We analyze firstly the singularities associated with "two-body collisions".

For notational convenience let us assume, without loss of generality, that the two particles involved in the two-body event - which happens at $\tau=\tau_{b}$ - carry the labels 1 and 2 , and let us call $a$ the coupling constant associated with this particle pair,

$$
a=a_{12}=a_{21} .
$$

Let us firstly assume that

$$
\operatorname{Re}(a)>0,
$$

so that the real part of the branch-point exponent $\gamma$ (see (4.6), and below),

$$
\gamma=1 /(1+a)
$$


satisfies the restriction

$$
0<\operatorname{Re}(\gamma)<1
$$

It can then be shown (see Appendix C) that in the neighborhood of a "two-body collision" occurring at $\tau=\tau_{b}$ the solution $\underline{\zeta}(\tau)$ of (1.7) features the following behavior:

$$
\begin{aligned}
\zeta_{s}(\tau)= & b+(-1)^{s-1} c\left(\tau-\tau_{b}\right)^{\gamma}+v\left(\tau-\tau_{b}\right) \\
& +\sum_{k=1}^{\infty} \sum_{l, m=0 ; l+m \geq 1}^{\infty} g_{k l m}^{(s)}\left(\tau-\tau_{b}\right)^{k+l \gamma+m(1-\gamma)}, \quad s=1,2, \\
\zeta_{n}(\tau)= & b_{n}+v_{n}\left(\tau-\tau_{b}\right) \\
& +\sum_{k=1}^{\infty} \sum_{l=\delta_{k 1}}^{\infty} \sum_{m=0}^{\infty} g_{k l m}^{(n)}\left(\tau-\tau_{b}\right)^{k+l \gamma+m(1-\gamma)}, \quad n=3, \ldots, N .
\end{aligned}
$$

In these formulas the $2 N$ (complex) constants $b, c, v, \tau_{b}$ and $b_{n}, v_{n}($ with $n=3, \ldots, N)$ are arbitrary, except for the inequalities

$$
b \neq b_{n}, \quad b_{n} \neq b_{m}, \quad n, m=3, \ldots, N
$$

while the coefficients of the sums are determined in terms of these constants (see Appendix C). Clearly these formulas, (5.4), entail $\zeta_{1}\left(\tau_{b}\right)=\zeta_{2}\left(\tau_{b}\right)=b \neq \zeta_{n}\left(\tau_{b}\right)=b_{n} \neq$ $\zeta_{m}\left(\tau_{b}\right)=b_{m}$ for $n \neq m, 3 \leq n, m \leq N$ with $\left|\zeta_{1}\left(\tau_{b}\right)\right|=\left|\zeta_{2}\left(\tau_{b}\right)\right|=|b|<\infty$ but (see (5.4a) and (15.2b) ) $\left|\zeta_{1}^{\prime}\left(\tau_{b}\right)\right|=\left|\zeta_{2}^{\prime}\left(\tau_{b}\right)\right|=\infty$, while $\left|\zeta_{n}\left(\tau_{b}\right)\right|=\left|b_{n}\right|<\infty$ and (see (5.4b) and (5.2b) $)\left|\zeta_{n}^{\prime}\left(\tau_{b}\right)\right|=\left|v_{n}\right|<\infty, n=3, \ldots, N$. This confirms that the corresponding event is to be interpreted as a "collision" of the two particles 1 and 2, with both colliding particles moving infinitely fast at the collision time $\tau=\tau_{b}$. What interests us most is the nature of the corresponding singularity: a branch point characterized by the exponents $\gamma$ and $1-\gamma$, see (5.3). And the fact that such a singularity is associated with an expression of the solution $\underline{\zeta}(\tau)$ of (1.7) that features, see (5.4), the maximal number, $2 N$, of arbitrary constants, demonstrates the generic character of such singularities, which are therefore likely to be featured by the solutions $\underline{\zeta}(\tau)$ corresponding to a generic set of initial data.

Of course a more complete treatment shall have to face the nontrivial task to prove that the iteration used in Appendix $\mathrm{C}$ to justify (5.4) does converge and thereby yields a solution which has indeed the analytic structure suggested by the formulas written above. In this paper we limit our consideration, both here and below, to this incomplete treatment, which is however sufficient to guess the character of the branch points.

Likewise, if the inequality (5.2a) is reversed,

$$
\operatorname{Re}(a)<0
$$

it can be shown (see Appendix C) that in the neighborhood of a "two-body collision" the behavior of the solutions $\underline{\zeta}(\tau)$ is characterized, rather than by (5.4), by the following 
expressions:

$$
\begin{aligned}
& \zeta_{1}(\tau)=b+c\left(\tau-\tau_{b}\right)^{1+\beta}+\sum_{k, l=1 ; k+l \geq 3}^{\infty} g_{k l}^{(1)}\left(\tau-\tau_{b}\right)^{k+l \beta}, \\
& \zeta_{2}(\tau)=b+v_{2}\left(\tau-\tau_{b}\right)-c\left(\tau-\tau_{b}\right)^{1+\beta}+\sum_{k=1}^{\infty} \sum_{l=2 \delta_{k 1}}^{\infty} g_{k l}^{(2)}\left(\tau-\tau_{b}\right)^{k+l \beta}, \\
& \zeta_{n}(\tau)=b_{n}+v_{n}\left(\tau-\tau_{b}\right)+\sum_{k=2}^{\infty} \sum_{l=0}^{\infty} g_{k l}^{(n)}\left(\tau-\tau_{b}\right)^{k+l \beta}, \quad n=3, \ldots, N,
\end{aligned}
$$

with

$$
\beta=-2 a
$$

so that (see (5.5a))

$$
\operatorname{Re}(\beta)>0 .
$$

In these formulas the $2 N$ (complex) constants $b, c, v_{2}, \tau_{b}$ and $b_{n}, v_{n}$ (with $n=3, \ldots, N$ ) are arbitrary (except, again, for the inequalities (5.4C)), while the coefficients of the sums are determined in terms of these constants (see Appendix $\mathrm{C}$ ). Clearly these formulas (see (5.6) and (5.4c)) entail again $\zeta_{1}\left(\tau_{b}\right)=\zeta_{2}\left(\tau_{b}\right)=b \neq \zeta_{n}\left(\tau_{b}\right)=b_{n} \neq \zeta_{m}\left(\tau_{b}\right)=b_{m}$ for $n \neq m, 3 \leq n, m \leq N$ with $\left|\zeta_{1}\left(\tau_{b}\right)\right|=\left|\zeta_{2}\left(\tau_{b}\right)\right|<\infty$, but now (see (5.6a) with (5.5b) and (5.6b) $) \zeta_{1}^{\prime}\left(\tau_{b}\right)=0, \zeta_{2}^{\prime}\left(\tau_{b}\right)=v_{2}$ (so that $\zeta_{1}^{\prime}\left(\tau_{b}\right) \zeta_{1}^{\prime}\left(\tau_{b}\right)=0$; see the right-hand side of (1.7)), while of course again $\left|\zeta_{n}\left(\tau_{b}\right)\right|=\left|b_{n}\right|<\infty$ and (see (5.2b) $)\left|\zeta_{n}^{\prime}\left(\tau_{b}\right)\right|=\left|v_{n}\right|<\infty$ for $n=3, \ldots, N$. Hence the corresponding event is again to be interpreted as a "collision" of the two particles 1 and 2, but now with particle 1 having zero velocity at the time of the collision, in contrast to particle 2 which moves with velocity $v_{2}$ (note the notational distinction thereby introduced among the two colliding particles); while for our purposes the interpretation of these formulas, $(5.6 \mathrm{a}, \mathrm{b}, \mathrm{c})$, is that the nature of the corresponding singularity is a branch point characterized by the exponent $\beta$, see (5.7). And again the fact that such a singularity is associated with an expression of the solution $\zeta_{n}(\tau)$ of (1.7) that features, see $(5.6 \mathrm{a}, \mathrm{b}, \mathrm{c})$, the maximal number, $2 N$, of arbitrary constants, demonstrates the generic character of such singularities, which are therefore likely to be featured by the solutions $\underline{\zeta}(\tau)$ corresponding to a generic set of initial data.

This concludes our discussion of the singularities associated with "two-body collisions". Those associated with "collisions" involving more than two particles can be discussed in an analogous manner (also taking advantage of the results of Section 3), but in view of their lack of genericity we forsake their treatment here, and we rather proceed to discuss the singularities associated with "escapes to infinity".

This phenomenon can only occur if, for some group of the interacting particles, which without loss of generality is hereafter assumed to be formed by the $M$ particles with labels from 1 to $M$ (where $2 \leq M \leq N$ ), the quantity $A$,

$$
A=(2 / M) \sum_{n, m=1 ; n>m}^{M} a_{n m},
$$


has real part less than negative unity,

$$
\operatorname{Re}(A)<-1,
$$

so that the corresponding quantity $\Gamma$ (see $(3.2 \mathrm{~b}))$,

$$
\Gamma=(1+A)^{-1}
$$

has negative real part,

$$
\operatorname{Re}(\Gamma)<0 .
$$

The quantities $a_{n m}$ appearing in the right-hand side of (5.8a) are of course the coupling constants that characterize the two-body interactions acting among the particles belonging to this group of $M$ particles, see (1.7) or (1.5); and there may of course be, for a given $N$-body problem, several subgroups of particles such that the corresponding quantity $\Gamma$, defined according to the above prescription, has negative real part, see (5.9b). Let us assume for simplicity that there is just one such group. It is then easily seen that the dominant term at $\tau \approx \tau_{b}$ of the solution $\underline{\zeta}(\tau)$ representing the "escape to infinity" of the $M$ coordinates $\zeta_{n}(\tau), n=1, \ldots, M$, reads

$$
\begin{aligned}
& \zeta_{n}(\tau) \approx c_{n}\left(\tau-\tau_{b}\right)^{\Gamma}, \quad n=1, \ldots, M, \\
& \zeta_{n}(\tau)=b_{n}, \quad n=M+1, \ldots, N .
\end{aligned}
$$

In these formulas the $N$ (complex) constants $b_{n}$ are arbitrary, while the coefficients $c_{n}$, $n=1, \ldots, M$ are determined (up to a common rescaling factor) in terms of the coupling constants $a_{n m}$ with $n, m=1, \ldots, M$ by (3.3a) with (5.8a). Clearly these formulas (see (5.10) and (5.8b) ) entail $\left|\zeta_{n}\left(\tau_{b}\right)\right|=\left|\zeta_{n}^{\prime}\left(\tau_{b}\right)\right|=\infty$ for $n=1, \ldots, M$ and $\zeta_{n}\left(\tau_{b}\right)=b_{n}$ for $n=M+1, \ldots, N$. Hence the corresponding "event" at $\tau=\tau_{b}$ is indeed to be interpreted as the "escape to infinity" of the $M$ particles with (conveniently chosen) labels from 1 to $M$. But, as already indicated in the introductory Section 1, a discussion of the analytic structure of this solution $\underline{\zeta}(\tau)$ at $\tau \approx \tau_{b}$ is postponed to a separate paper.

\section{Analysis of various motions}

In this section we survey the implications of the findings discussed above (especially in Section 5) for the motions of the physical models (1.1) characterized by various choices of the number $N$ of particles and of the coupling constants $a_{n m}$. We always refer, for notational convenience, to the complex avatar (1.5) of these equations of motion, and we display the trajectories of solutions produced via a computer code manufactured by one of us (MS) for the numerical integration of these equations of motion, (1.5). Additional numerical examples, as well as the discussion and display of the numerical code, will be reported elsewhere [8], enabling thereby any interested party to enjoy an ampler personal exploration of analogous numerical results (easily generated on a personal computer).

The basic mode of presentation of our results begins by choosing a specific model, characterized by the number $N$ of particles (for simplicity we restrict consideration below

to three- and four-body cases, $N=3$ and $N=4$, although the simulation program can 
actually handle an arbitrary number $N$ of moving points) and by a set of coupling constants $a_{n m}$, see (1.5), which we always assume to satisfy the symmetry property (1.12). We moreover always set, for notational convenience (and of course without loss of generality, see the Remark 1.1)

$$
\omega=2 \pi
$$

entailing an assignment of the time scale such that the basic period be unity,

$$
T=1
$$

see (1.2). We then generally consider, for each model, a sequence of solutions of the equations of motions (1.5) corresponding to a sequence of initial data characterized by the same initial configuration of the particle positions in the plane and by sets of initial velocities modified, from one solution to the next one considered, via multiplication by an increasing sequence of positive factors $\mu$ (by analogy with the notation in (1.10b)):

$$
z_{n}(0)=z_{n}^{(0)}, \quad \dot{z}_{n}(0)=\mu \dot{z}_{n}^{(0)} .
$$

As implied by the treatment of the preceding sections (see in particular Section 1 and the Remarks 1.1 and 1.3) this entails that all the sequentially analyzed solutions $\underline{z}(t)$ of the "physical" equations of motion (in their avatar (1.5), and characterized by a specific assignment of $N$ and $a_{n m}$ ) correspond, via the fundamental relation (1.6) (with an appropriately chosen value of the constant $\omega$ in this formula (1.6), namely $\omega=2 \pi / \mu$ ), to the same solution $\underline{\zeta}(\tau)$ of $(1.7)$, characterized by the same values of $N$ and $a_{n m}$, and by the same initial data,

$$
\zeta_{n}(0)=z_{n}^{(0)}, \quad \zeta_{n}^{\prime}(0)=\dot{z}_{n}^{(0)}
$$

hence the solutions $\underline{z}(t)$ of the equations of motion (1.5) discussed below are obtained via (1.6) from the same solution $\underline{\zeta}(\tau)$ of $(1.7)$ by traveling on the Riemann surface associated with this solution of (1.7) on circular contours $\tilde{C}=\tilde{C}(\mu)$, in the complex $\tau$-plane, the diameters of which always lie on the upper imaginary axis of the complex $\tau$-plane, with their lower ends fixed at the origin, $\tau=0$, while the size of these diameters is $\mu / \pi$, namely it gets increased via multiplication by the same rescaling factor, $\mu$, that yielded by multiplication the set of initial velocities. The sequence of solutions we consider starts generally with a completely periodic one having the basic period $T=1$; note that, as implied by Proposition 1.2. and as of course confirmed by the numerical simulations, such solutions $\underline{z}(t)$ always exist for sufficiently small initial velocities, namely sufficiently small values of $\mu$ (they correspond to solutions $\underline{\zeta}(\tau)$ of $(1.7)$ which are singularity free, on the main sheet of their Riemann surface, inside the circle $\tilde{C}(\mu)$ - which, for very small $\mu$, becomes very small, and gets very close to the origin, $\tau=0$, where the solution $\underline{\zeta}(\tau)$ is of course holomorphic). The sequence of solutions $\underline{z}(t)$ identified according to the procedure indicated above corresponds therefore generally to the sequential coming into play of different branch points (see Section 5) of the same solution $\underline{\zeta}(\tau)$ of $(1.7)$, as they get enclosed inside the circular contours $\tilde{C}=\tilde{C}(\mu)$ when the size of these contours gets enlarged proportionally to the parameter $\mu$ (which is sequentially increased from one simulation to the next one). The inclusion of such branch points may entail the transition - for models with 
real rational coupling constants, to which our consideration is mainly restricted below, as they display the most interesting phenomenology (aside from the fact that, in numerical computations, all numbers are rational!) - to solutions which are again completely periodic but with larger periods, or which possibly are no more periodic (although of course it is hardly possible to distinguish numerically a nonperiodic solution from one which is periodic but with a very large period, see below), according to a phenomenology which can be understood on the basis of the treatment of the preceding Sections 4 and 5 and the discussion at the end of Section 1 - an analysis which turns out to be indeed validated by the numerical results reported below (and see also the analogous discussion in Ref. [9]). We also present below some results with complex coupling constants, and tersely discuss the associated phenomenology. On the other hand, as already mentioned above, our consideration is restricted to coupling constants the real part of which is positive, $\operatorname{Re}\left(a_{n m}\right)>0$.

Enough of preliminary remarks: let us proceed to display and comment a few representative examples. In the graphs displayed below, particle 1 will be shown in Red, particle 2 in Green, particle 3 in Blue and particle 4 (if present) in Yellow. Whenever we felt such an additional indication might be usefully displayed we indicated with a black diamond the initial position of each particle (at $t=0$ ), with a black dot the position at a subsequent time $t=t_{1}$, and with smaller black dots the position at every subsequent integer multiple of $t_{1}$ (namely at $t=t_{k}=k t_{1}, k=2,3, \ldots$ ); in this manner the direction of the motion along the trajectories can be inferred (from the relative positions along the trajectories of the diamond and the larger dot), as well as some indication of the positions of the particles over time, as they move (by counting the dots along the trajectory). Of course a much more satisfactory visualization of the behavior of the many-body system is provided by simulations in which the particle motions are displayed as they unfold over time (as in a movie); it is planned to make available soon, via the web, the numerical code suitable to perform such simulations on personal computers [8]. Let us emphasize that such simulations are particularly stunning to watch in the case of high-period trajectories, which are very complicated (see below), so that the fact that the particles return eventually exactly on their tracks appears quite miraculous and is indeed a remarkable proof of the reliability of the numerics.

The first example we report is characterized by the following parameters:

$$
\begin{aligned}
& N=3 ; \quad a_{12}=a_{21}=a_{R G}=1, \quad a_{13}=a_{31}=a_{R B}=1 / 2, \\
& a_{23}=a_{32}=a_{G B}=3 / 2,
\end{aligned}
$$

and by the following values of the parameters $x_{n}^{(0)}, y_{n}^{(0)}, \dot{x}_{n}^{(0)}, \dot{y}_{n}^{(0)}$ characterizing the initial data via (6.2):

$$
\begin{array}{llll}
x_{1}^{(0)}=0, & y_{1}^{(0)}=0, & \dot{x}_{1}^{(0)}=-1, & \dot{y}_{1}^{(0)}=-0.5 ; \\
x_{2}^{(0)}=1, & y_{2}^{(0)}=0, & \dot{x}_{2}^{(0)}=0.5, & \dot{y}_{2}^{(0)}=1 ; \\
x_{3}^{(0)}=2, & y_{3}^{(0)}=0, & \dot{x}_{3}^{(0)}=0.5, & \dot{y}_{3}^{(0)}=-1 .
\end{array}
$$

Our numerical findings are synthesized in the Tables $6.1 \mathrm{a}, \mathrm{b}$, and in the sequence of Figures 6.1 (the contents of which are identified in Table 6.1a). Here and below we employ the acronym HSL (Hic Sunt Leones) to denote a (presumably) chaotic behavior. We trust 
the significance of this material - as presented here and in the rest of this section - to be self-evident, but we nevertheless add a few comments.

Table $6.1 \mathrm{a}$

\begin{tabular}{|c|c|c|c|c|c|c|c|c|c|c|c|}
\hline$\mu$ & 0.8 & 1 & 1.301 & 1.302 & 5.28 & 5.29 & 5.93 & 5.94 & 6.82 & 6.83 & 50 \\
\hline Period & 1 & 1 & 1 & 2 & 2 & 6 & 6 & 7 & 7 & HSL & HSL \\
\hline Fig. 6.1 & $\mathrm{a}$ & - & $\mathrm{b}$ & $\mathrm{c}$ & $\mathrm{d}$ & $\mathrm{e}$ & $\mathrm{f}$ & $\mathrm{g}$ & $\mathrm{h}$ & $\mathrm{i}$ & - \\
\hline
\end{tabular}

Table $6.1 \mathrm{~b}$

\begin{tabular}{|c|c|c|c|c|}
\hline Period Change & $1 \rightarrow 2$ & $2 \rightarrow 6$ & $6 \rightarrow 7$ & $7 \rightarrow$ HSL \\
\hline Colliding Particles & $\mathrm{R}-\mathrm{G}$ & $\mathrm{G}-\mathrm{B}$ & $\mathrm{R}-\mathrm{G}$ & $\mathrm{R}-\mathrm{B}$ \\
\hline Collision Time & 0.80 & 0.77 & 2.87 & 3.72 \\
\hline
\end{tabular}

Clearly for $\mu \leq 1.301$ the solution $\underline{\zeta}(\tau)$ of $(1.7)$ (with (6.3) and (6.4b) is holomorphic inside the circle $\tilde{C}(\mu)$, hence the (corresponding) solution $\underline{z}(t)$ of (1.5) (with (6.1), (6.2) and $(6.3 \mathrm{~b})$ ) is completely periodic with period $T=1$ (see the first 3 columns of Table $6.1 \mathrm{a}$, and Figs. 6.1a,b). For $1.302 \leq \mu \leq 5.28$ this (same) solution $\underline{\zeta}(\tau)$ of $(1.7)$ (with (6.3) and (6.4b) has a single singularity inside the circle $\tilde{C}(\mu)$, a branch point with exponent

$$
\gamma_{R G}=1 /\left(a_{R G}+1\right)=1 / 2
$$

hence by traveling on the Riemann surface associated with $\underline{\zeta}(\tau)$ on the circle $\tilde{C}(\mu)$ two sheets are now visited before returning to the point of departure, and therefore the (corresponding) solution $\underline{z}(t)$ of $(1.5)$ (with (6.1), (6.2) and (6.3b)) is completely periodic with period $T=2$ (see the fourth and fifth columns of Table 6.1a, and Figs. 6.1c,d). The transition from the regime with period $T=1$ to that with period $T=2$ occurs due to the entrance of the relevant branch point from outside to inside the circle $\tilde{C}(\mu)$; this corresponds to the occurrence of a collision among the Red and Green particles (see Figs. 6.1b and $6.1 \mathrm{c}$, and the first column of Table 6.1b), consistently - according to the analysis of Section 5 - with the presence of the coupling constant $a_{R G}=1$ in (6.5a).

For $5.29 \leq \mu \leq 5.93$ this (same) solution $\underline{\zeta}(\tau)$ of (1.7) (with (6.3) and (6.4b)) has two singularities inside the circle $\tilde{C}(\mu)$, namely the branch point with the exponent (6.5a) and a second one (located either on the main sheet, or possibly on the second sheet accessed via the cut associated with the first branch point, with exponent (6.5a)), with exponent

$$
\gamma_{G B}=1 /\left(a_{G B}+1\right)=2 / 5,
$$

which opens the way to four additional sheets; hence by traveling on the Riemann surface associated with $\underline{\zeta}(\tau)$ on the circle $\tilde{C}(\mu)$ altogether six sheets are now visited before returning to the point of departure, and therefore the (corresponding) solution $\underline{z}(t)$ of $(1.5)$ (with (6.1), (6.2) and (6.3b)) is completely periodic with period $T=6$ (see the sixth and seventh columns of Table 6.1a, and Figs. 6.1e,f). The transition from the regime with period $T=2$ to that with period $T=6$ occurs due to the entrance (in one of the two sheets of the Riemann surface) of the (second) branch point, characterized by the exponent (6.5b), from outside to inside the circle $\tilde{C}(\mu)$; this corresponds to the occurrence of a collision among the Green and Blue particles (see Figs. 6.1d and 6.1e, and the second column of Table $6.1 \mathrm{~b}$ ), consistently - again according to the analysis of Section 5 - with the presence of the coupling constant $a_{G B}=3 / 2$ in (6.5b). 
Another transition occurs for a value of $\mu$ between 5.93 and 5.94 (see the seventh and eighth columns of Table 6.1, and Figs. 6.1f,g), associated to another collision among the Red and Green particles (see the third column of Table 6.2) and bringing inside the circle $\tilde{C}(\mu)$ another branch point of square-root type (see (6.5a)), that opens the way to one additional sheet of the Riemann surface, and thereby causes the solutions $\underline{z}(t)$ of $(1.5)$ (with (6.1), (6.2) and (6.3b)) to be completely periodic with period $T=7$ (see the eighth and ninth columns of Table 6.1a, and Figs. 6.1g,h) for $5.94 \leq \mu \leq 6.82$.

Finally another collision occurs, for $\mu$ between 6.82 and 6.83 (see the ninth and tenth column of Table 6.1a, and Figs. 6.1h,i), among the Blue and Red particles (see the last column of Table $6.1 \mathrm{~b}$ ), which brings (on one of the seven relevant sheets of the Riemann surface associated with the solution $\underline{\zeta}(\tau)$ of $(1.7)$ with (6.3) and (6.4b) ) a branch point with exponent

$$
\gamma_{R B}=1 /\left(1+a_{R B}\right)=2 / 3
$$

inside the circle $\tilde{C}(\mu)$. This opens the way to two additional sheets of the Riemann surface (associated with the solution $\underline{\zeta}(\tau)$ of (1.7) with (6.3) and (6.4b)); but clearly on (at least) one of these two sheets there is (at least) one additional branch point (characterized by one of the three exponents $(6.5 \mathrm{a}, \mathrm{b}, \mathrm{c}))$ which is already located inside the circle $\tilde{C}(\mu)$, and this opens the way to additional sheets on (some of) which there also are additional branch points inside the circle $\tilde{C}(\mu)$, and so on and on, causing presumably a transition to chaos (see the last two columns of Table 6.1a, and Fig. 6.1i) according to the mechanism outlined at the end of the introductory Section 1 and discussed in Section 4 (and also discussed, in an analogous context, in Ref. [9]).

The second example we report is much richer. It is characterized by the following parameters:

$$
\begin{aligned}
& N=3 ; \quad a_{12}=a_{21}=a_{R G}=1, \quad a_{13}=a_{31}=a_{R B}=2, \\
& a_{23}=a_{32}=a_{G B}=3,
\end{aligned}
$$

and by the following values of the parameters $x_{n}^{(0)}, y_{n}^{(0)}, \dot{x}_{n}^{(0)}, \dot{y}_{n}^{(0)}$ characterizing the initial data via (6.2):

$$
\begin{aligned}
& x_{1}^{(0)}=0, \quad y_{1}^{(0)}=0, \quad \dot{x}_{1}^{(0)}=-1, \quad \dot{y}_{1}^{(0)}=1 ; \\
& x_{2}^{(0)}=0, \quad y_{2}^{(0)}=1, \quad \dot{x}_{2}^{(0)}=1, \quad \dot{y}_{2}^{(0)}=0 \text {; } \\
& x_{3}^{(0)}=-1, \quad y_{3}^{(0)}=0, \quad \dot{x}_{3}^{(0)}=-0.5, \quad \dot{y}_{3}^{(0)}=-0.5 \text {. }
\end{aligned}
$$

Our numerical findings are now synthesized in the Tables 6.2a,b, and in the sequence of Figs. 6.2 (the contents of which are identified in Table 6.2a). We urge the alert reader to repeat the analysis we detailed in the preceding example: the fact that the branch point exponents take in this case the values $\gamma_{R G}=1 / 2, \gamma_{B R}=1 / 3$ and $\gamma_{G B}=1 / 4$ is of course relevant, and clearly some transitions from a periodic regime to another periodic regime with higher period are the effect of a single identifiable branch point, while others are due to the synergistic effect of several branch points coming into play simultaneously. The presence of trajectories with stunningly large periods is of course noteworthy. 
Table $6.2 \mathrm{a}$

\begin{tabular}{|c|c|c|c|c|c|c|c|c|c|}
\hline$\mu$ & 0.5 & 0.780 & 0.781 & 1 & 1.213 & 1.214 & 1.219 & 1.220 & 1.241 \\
\hline Period & 1 & 1 & 6 & 6 & 6 & 49 & 49 & 50 & 50 \\
\hline Fig. 6.2 & $\mathrm{a}$ & $\mathrm{b}$ & $\mathrm{c}$ & - & $\mathrm{d}$ & $\mathrm{e}$ & - & - & - \\
\hline
\end{tabular}

\begin{tabular}{|c|c|c|c|c|c|c|c|c|c|}
\hline$\mu$ & 1.242 & 1.25 & 1.293 & 1.294 & 1.295 & 1.400 & 1.401 & 1.41 & 1.442 \\
\hline Period & 51 & 51 & 51 & 57 & 58 & 58 & 59 & 59 & 59 \\
\hline Fig. 6.2 & - & $\mathrm{f}$ & - & $\mathrm{g}$ & - & - & - & $\mathrm{h}$ & - \\
\hline
\end{tabular}

\begin{tabular}{|c|c|c|c|c|c|c|c|c|c|}
\hline$\mu$ & 1.443 & 1.721 & 1.722 & 1.8 & 1.944 & 1.945 & 2.053 & 2.054 & 2.1 \\
\hline Period & 65 & 65 & 66 & 66 & 66 & 68 & 68 & 71 & 71 \\
\hline Fig. 6.2 & - & - & - & $\mathrm{i}$ & - & - & - & - & $\mathrm{j}$ \\
\hline
\end{tabular}

\begin{tabular}{|c|c|c|c|c|c|c|c|c|c|}
\hline$\mu$ & 2.108 & 2.109 & 2.164 & 2.165 & 2.17 & 2.171 & 2.172 & 2.2 & 2.5 \\
\hline Period & 71 & 72 & 72 & 74 & 74 & 74 & HSL & HSL & HSL \\
\hline Fig. 6.2 & - & - & - & - & $\mathrm{k}$ & - & - & $\mathrm{m}$ & - \\
\hline
\end{tabular}

Table $6.2 \mathrm{~b}$

\begin{tabular}{|c|c|c|c|c|c|}
\hline Period Change & $1 \rightarrow 6$ & $6 \rightarrow 49$ & $49 \rightarrow 50$ & $50 \rightarrow 51$ & $51 \rightarrow 57$ \\
\hline Colliding Particles & $\mathrm{R}-\mathrm{B}$ & $\mathrm{R}-\mathrm{G}$ & $\mathrm{R}-\mathrm{G}$ & $\mathrm{R}-\mathrm{G}$ & $\mathrm{R}-\mathrm{G}$ \\
\hline Collision Time & 0.48 & 3.54 & 24.72 & 20.59 & 43.75 \\
\hline
\end{tabular}

\begin{tabular}{|c|c|c|c|c|c|}
\hline Period Change & $57 \rightarrow 58$ & $58 \rightarrow 59$ & $59 \rightarrow 65$ & $65 \rightarrow 66$ & $66 \rightarrow 68$ \\
\hline Colliding Particles & $\mathrm{R}-\mathrm{G}$ & $\mathrm{R}-\mathrm{G}$ & $\mathrm{R}-\mathrm{G}$ & $\mathrm{R}-\mathrm{G}$ & $\mathrm{R}-\mathrm{B}$ \\
\hline Collision Time & 31.49 & 27.37 & 8.35 & 44.25 & 54.41 \\
\hline
\end{tabular}

\begin{tabular}{|c|c|c|c|c|}
\hline Period Change & $68 \rightarrow 71$ & $71 \rightarrow 72$ & $72 \rightarrow 74$ & $74 \rightarrow \mathrm{HSL}$ \\
\hline Colliding Particles & $\mathrm{G}-\mathrm{B}$ & $\mathrm{R}-\mathrm{G}$ & $\mathrm{R}-\mathrm{B}$ & $\mathrm{R}-\mathrm{G}$ \\
\hline Collision Time & 56.26 & 5.63 & 13.75 & 24.905 \\
\hline
\end{tabular}

The third example we report is the last one with coupling constants which are both real and rational - namely the last one belonging to the class that can give rise to completely periodic motions with periods larger than unity; and it features four particles. It is characterized by the following parameters:

$$
\begin{aligned}
& N=4 ; \quad a_{12}=a_{21}=a_{R G}=2, \quad a_{13}=a_{31}=a_{R B}=5 / 2, \\
& a_{14}=a_{41}=a_{R Y}=1, \quad a_{23}=a_{32}=a_{G B}=1 / 2, \quad a_{24}=a_{42}=a_{G Y}=3, \\
& a_{34}=a_{43}=a_{B Y}=3 / 2,
\end{aligned}
$$

and by the following values of the parameters $x_{n}^{(0)}, y_{n}^{(0)}, \dot{x}_{n}^{(0)}, \dot{y}_{n}^{(0)}$ characterizing the initial data via (6.2):

$$
\begin{array}{llll}
x_{1}^{(0)}=0, & y_{1}^{(0)}=1, & \dot{x}_{1}^{(0)}=1, & \dot{y}_{1}^{(0)}=-1 ; \\
x_{2}^{(0)}=0, & y_{2}^{(0)}=0, & \dot{x}_{2}^{(0)}=-0.5, & \dot{y}_{2}^{(0)}=0 ; \\
x_{3}^{(0)}=-1.5, & y_{3}^{(0)}=0, & \dot{x}_{3}^{(0)}=0, & \dot{y}_{3}^{(0)}=-0.5 ; \\
x_{4}^{(0)}=1, & y_{4}^{(0)}=0, & \dot{x}_{4}^{(0)}=-1, & \dot{y}_{4}^{(0)}=1 .
\end{array}
$$

The corresponding numerical results are synthesized in the Tables $6.3 \mathrm{a}, \mathrm{b}$, and in the sequence of Figs. 6.3 (the contents of which are identified in Table 6.3a). We again urge 
the alert reader to repeat the analysis we detailed in the first example, taking of course into account that, corresponding to the six different coupling constants (see (6.7a)), there are in this case six different branch point exponents: $\gamma_{R G}=1 / 3, \gamma_{B R}=2 / 7, \gamma_{R Y}=1 / 2$, $\gamma_{G B}=2 / 3, \gamma_{G Y}=1 / 4$ and $\gamma_{B Y}=2 / 5$. And the presence of trajectories with remarkably large periods should again be noted.

Table $6.3 \mathrm{a}$

\begin{tabular}{|c|c|c|c|c|c|c|c|c|}
\hline$\mu$ & 0.4 & 0.626 & 0.627 & 0.692 & 0.693 & 1 & 1.037 & 1.038 \\
\hline Period & 1 & 1 & 4 & 4 & 6 & 6 & 6 & 22 \\
\hline Fig. 6.3 & $\mathrm{a}$ & $\mathrm{b}$ & $\mathrm{c}$ & $\mathrm{d}$ & $\mathrm{e}$ & - & $\mathrm{f}$ & $\mathrm{g}$ \\
\hline
\end{tabular}

\begin{tabular}{|c|c|c|c|c|c|c|c|c|c|}
\hline$\mu$ & 1.156 & 1.157 & 1.207 & 1.208 & 1.3 & 1.318 & 1.319 & 1.32 & 1.4 \\
\hline Period & 22 & 23 & 23 & 26 & 26 & 26 & HSL & HSL & HSL \\
\hline Fig. 6.3 & - & - & - & - & $\mathrm{h}$ & - & - & $\mathrm{i}$ & - \\
\hline
\end{tabular}

Table $6.3 \mathrm{~b}$

\begin{tabular}{|c|c|c|c|c|c|c|}
\hline Period Change & $1 \rightarrow 4$ & $4 \rightarrow 6$ & $6 \rightarrow 22$ & $22 \rightarrow 23$ & $23 \rightarrow 26$ & $26 \rightarrow \mathrm{HSL}$ \\
\hline Colliding Particles & $\mathrm{G}-\mathrm{Y}$ & $\mathrm{R}-\mathrm{G}$ & $\mathrm{R}-\mathrm{B}$ & $\mathrm{R}-\mathrm{Y}$ & $\mathrm{G}-\mathrm{Y}$ & $\mathrm{B}-\mathrm{Y}$ \\
\hline Collision Time & 0.44 & 3.33 & 3.33 & 9.26 & 12.36 & 14.53 \\
\hline
\end{tabular}

Finally let us display two examples with complex coupling constants, which can both be considered deformations of the first example considered above, obtained by adding to the three coupling constants characterizing that model, see (6.4a), (not too large) imaginary parts, either all of them negative (first example below), or all of them positive (second example below). We also take, in both cases, the same assignment (6.4b) of the parameters that determine the initial data via (6.2).

The first of these last two examples is characterized by the parameters

$$
\begin{aligned}
& N=3 ; \quad a_{12}=a_{21}=a_{R G}=1-0.3 i \\
& a_{13}=a_{31}=a_{R B}=0.5-0.4 i, \quad a_{23}=a_{32}=a_{G B}=1.5-0.2 i
\end{aligned}
$$

and the corresponding trajectories are displayed in the sequence of Figs. 6.4a-f; in each figure the value is now indicated of the scaling factor $\mu$ that determines via (6.2) with (6.4b) the initial data for the displayed motions, as well as the period - which is of course always $T=1$ - in the case of periodic motions (see Figs. 6.4a,b) or instead the final time $t$ of the displayed trajectories in the case of nonperiodic motions (see Figs. 6.4c-f).

The qualitative understanding of these results is easy, and it displays an interesting phenomenology - limit cycles, with (asymptotic) period $T=1$ - that is presumably characteristic of all models of type (1.5) with complex coupling constants the imaginary parts of which are negative, $\operatorname{Im}\left(a_{n m}\right)<0$. Indeed the (first) two Figs. 6.4a,b display the completely periodic motions, with period $T=1$, which as we know are always featured by all models of type (1.5), as guaranteed by Proposition [1.2 and let us recall that our understanding of the existence of these completely periodic solutions $\underline{z}(t)$ of (1.5) with period $T=1$ is in terms of the corresponding solution $\underline{\zeta}(\tau)$ of (1.7) being singularity free inside the corresponding circles $\tilde{C}(\mu)$ in the complex $\tau$-plane. We also know that, by increasing $\mu$ - hence, by increasing the size of the circle $\tilde{C}(\mu)$, of diameter $\mu / \pi-$ branch points (of the corresponding solution $\underline{\zeta}(\tau)$ of $(1.7)$ ) are eventually enclosed inside it. These branch points are characterized by exponents $\gamma_{n m}=1 /\left(1+a_{n m}\right)$, hence by 
exponents that, for this example, have a positive imaginary part, $\operatorname{Im}\left(\gamma_{n m}\right)>0$, since the imaginary part of the coupling constant $a_{n m}$ is negative, $\operatorname{Im}\left(a_{n m}\right)<0$. In this case the main effect of such a branch point, when one travels over the circle $\tilde{C}(\mu)$ namely follows the evolution of $\underline{\zeta}(\tau)$ with $\tau$ given by the formula $\tau=\mu[\exp (2 \pi i t)-1] /(2 \pi i)$ (see $(1.6)$ and $(6.1))$, is to yield the shrinking factor $\exp \left[-2 \pi \operatorname{Im}\left(\gamma_{n m}\right) t\right]$, as entailed by the formula

$$
\begin{gathered}
\left(\tau-\tau_{b}\right)^{\gamma_{n m}}=\left\{\mu[\exp (2 \pi i t)-1] /(2 \pi i)-\tau_{b}\right\}^{\gamma_{n m}}=\exp \left[-2 \pi \operatorname{Im}\left(\gamma_{n m}\right) t\right] \\
\times \exp \left[2 \pi i \operatorname{Re}\left(\gamma_{n m}\right) t\right]\left\{\mu[1-\exp (-2 \pi i t)] /(2 \pi i)-\tau_{b} \exp (-2 \pi i t)\right\} .
\end{gathered}
$$

The effect of such shrinking, as long as it is relevant, is to reduce - qualitatively - by the same amount the magnitude of the (nonlinear) right-hand side of the equations of motion (1.5) or (1.7); but we know (see Section 1) that when this happens eventually one reaches the regime characterized by the completely periodic solutions of period $T=1$ of (1.5), the existence of which is guaranteed by Proposition [1.2. This is what one sees in the trajectories displayed in the Figs. (6.4c-f): in fact, given the relative scale of the motions of the various particles, what one apparently sees is that one of the three particles eventually stops (asymptotically; or it might actually tend to a periodic trajectory of very small size, moving along it very slowly since the period of that asymptotic motion is always $T=1$; in either case, because of its slow motion, it essentially decouples hence moves on a circular orbit, neither feeling the presence of the other particles nor affecting their motion, due to the velocity-dependence of the two-body forces, see the right-hand side of (1.5)), while the other two particles approach asymptotically (in fact, given the graphical limitations, seem to eventually follow) periodic trajectories, always of course with period $T=1$. Specifically, the first transition from the completely periodic regime to that affected by the presence of a branch point occurs at a value of $\mu$ between 1.682 and 1.683, due to a collision among the Red and Green particles occurring approximately at $t=0.85$ (see Figs. $6.4 \mathrm{~b}, \mathrm{c}$; note that the latter of these two figures displays a motion in which the Green and Blue particles follow asymptotically periodic trajectories, while the Red particle seems to stop asymptotically). Several other transitions occur for larger values of $\mu$. A representative instance, due to a collision among the Green and Blue particles occurring approximately at $t=0.85$, is displayed by Figs. 6.4 d,e: note that Fig. 6.4d displays a motion in which asymptotically all three particles move on periodic trajectories, but the Red one moves on a circle of very small size; while Fig. 6.4e displays as well a motion with all three particles asymptotically on periodic trajectories, but now with the Blue one approaching asymptotically a circular trajectory of very small size, in fact so small that it appears merely as a point in the figure. Finally Fig. 6.4f displays a motion in which the Blue particle seems to stop asymptotically, while the other two approach periodic trajectories.

The interpretation of this phenomenology as outlined above is confirmed by the numerical results of the last example we report, in which the imaginary parts of the coupling constants $a_{n m}$ are instead positive, $\operatorname{Im}\left(a_{n m}\right)>0$. This example is characterized by the parameters

$$
\begin{aligned}
& N=3 ; \quad a_{12}=a_{21}=a_{R G}=1+0.4 i, \\
& a_{13}=a_{31}=a_{R B}=0.5+0.3 i, \quad a_{23}=a_{32}=a_{G B}=1.5+0.2 i,
\end{aligned}
$$

and the corresponding trajectories are displayed in the sequence of Figs. 6.5a-f; as above, in each figure the corresponding value is indicated of the scaling factor $\mu$ that determines 
the initial data via (6.2) with (6.4b), as well as the period - which is of course always $T=1$ - in the case of periodic motions (see Figs. 6.5a,b) or instead the final time $t$ of the displayed trajectories in the case of nonperiodic motions (see Figs. 6.5c-f). As in the preceding example, the completely periodic motions of period $T=1$ displayed in the first two figures correspond to the solutions predicted by Proposition 1.2, namely they correspond to the solution $\underline{\zeta}(\tau)$ of $(1.7)$ being singularity-free inside $\tilde{C}(\mu)$; while the behavior (spiraling outwardly) displayed by the other four figures is consistent with the coming into play of an expanding factor, the origin of which can be explained exactly as above (see (6.9)), the difference among expanding and shrinking behavior being of course consistent with the change of sign of the imaginary parts of the coupling constants $a_{n m}$. Specifically one sees from Figs. 6.5b,c that the first transition from the completely periodic regime is due to a collision among the Red and Green particles (occurring approximately at $t=0.74$ ), and another transition is apparent from Figs. 6.5d,e, due a collision among the Green and Blue particles (occurring approximately at $t=0,69$ ). Several other transitions occur between these, as well as for values of $\mu$ between 1.010 (see Fig. 6.5c) and 3.474 (see Fig. 6.5d), between 3.475 (see Fig. 6.5e) and 20 (see Fig. 6.5f), and beyond 20. Note that in all these simulations - with $\mu \geq 1.010$, namely when the motions are no more periodic - all three particles eventually spiral out. This, however, does not seem to represent a universal behavior; other (three-body) cases have been observed, with all coupling constants having positive imaginary parts, in which only two particles spiral out asymptotically, while the third one seems to stop. Clearly a more complete analysis of this phenomenology will have to be made; indeed while the discussion of the last two examples seems to provide a satisfactory qualitative understanding of the numerical results reported, it also confirms the richness of behaviors displayed by the many-body problem in the plane (1.1), or equivalently (1.5), leaving an ample scope for additional study (for instance, of cases with coupling constant some of which have positive, and others negative, imaginary parts) - and of course there is moreover the case with coupling constants having a negative real part, the investigation of which shall be reported separately.

Let us finally emphasize that the findings reported above are merely a tiny subset selected on the basis of their representative character - of the data we collected in our numerical explorations. Indeed we expect that every interested reader will eventually like to engage in personal experimentations, as soon as the computer program [8] which yielded these results shall become available (it is planned to put it on the web soon). In particular we recommend once again as a thrilling experience the observation as they evolve over time of periodic motions with large periods, such as some of those which have been displayed above (but only in the guise of trajectories: see for instance Figs. 6.1e-h and especially Figs. 6.2c-k, 6.3c-h). 

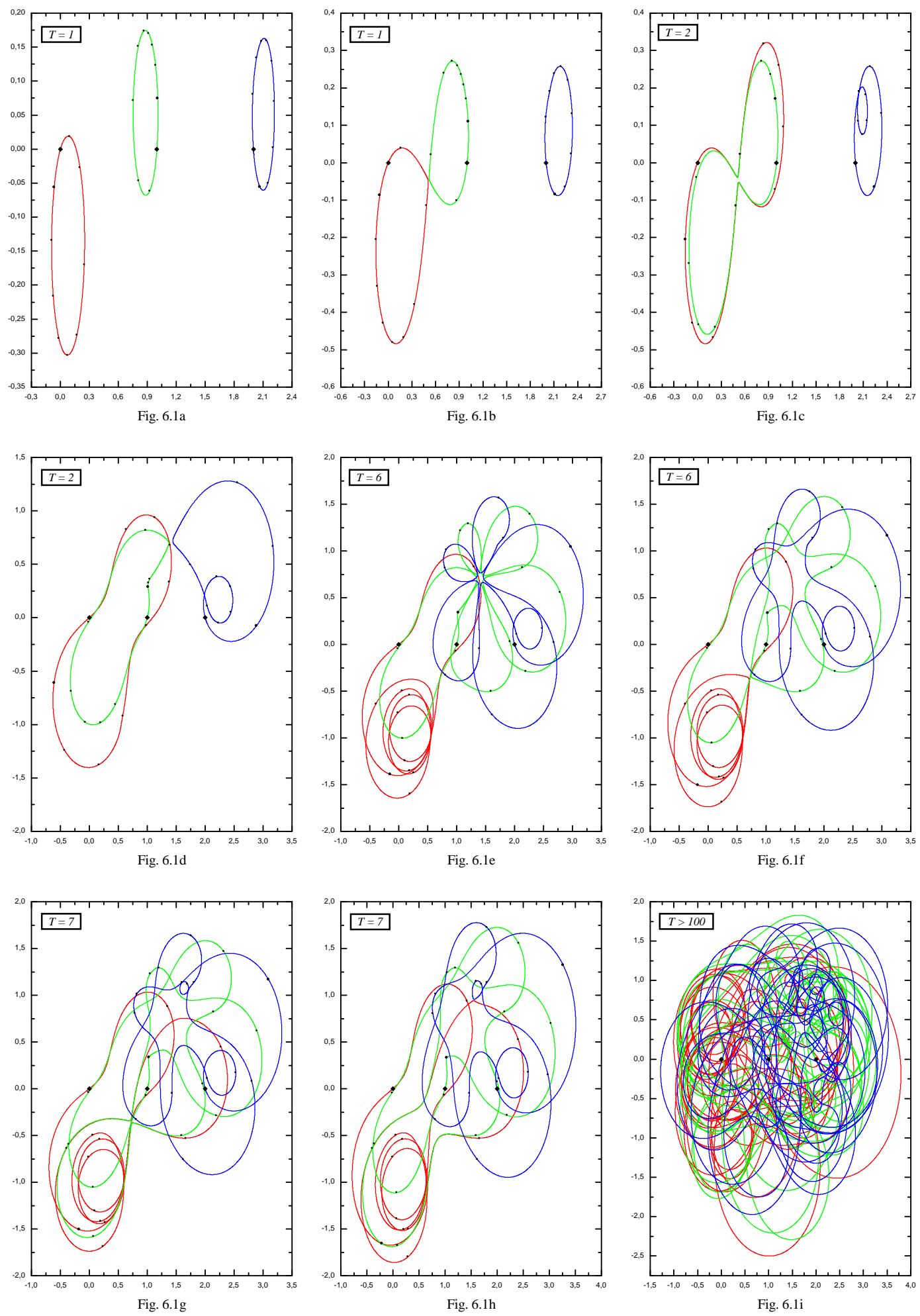

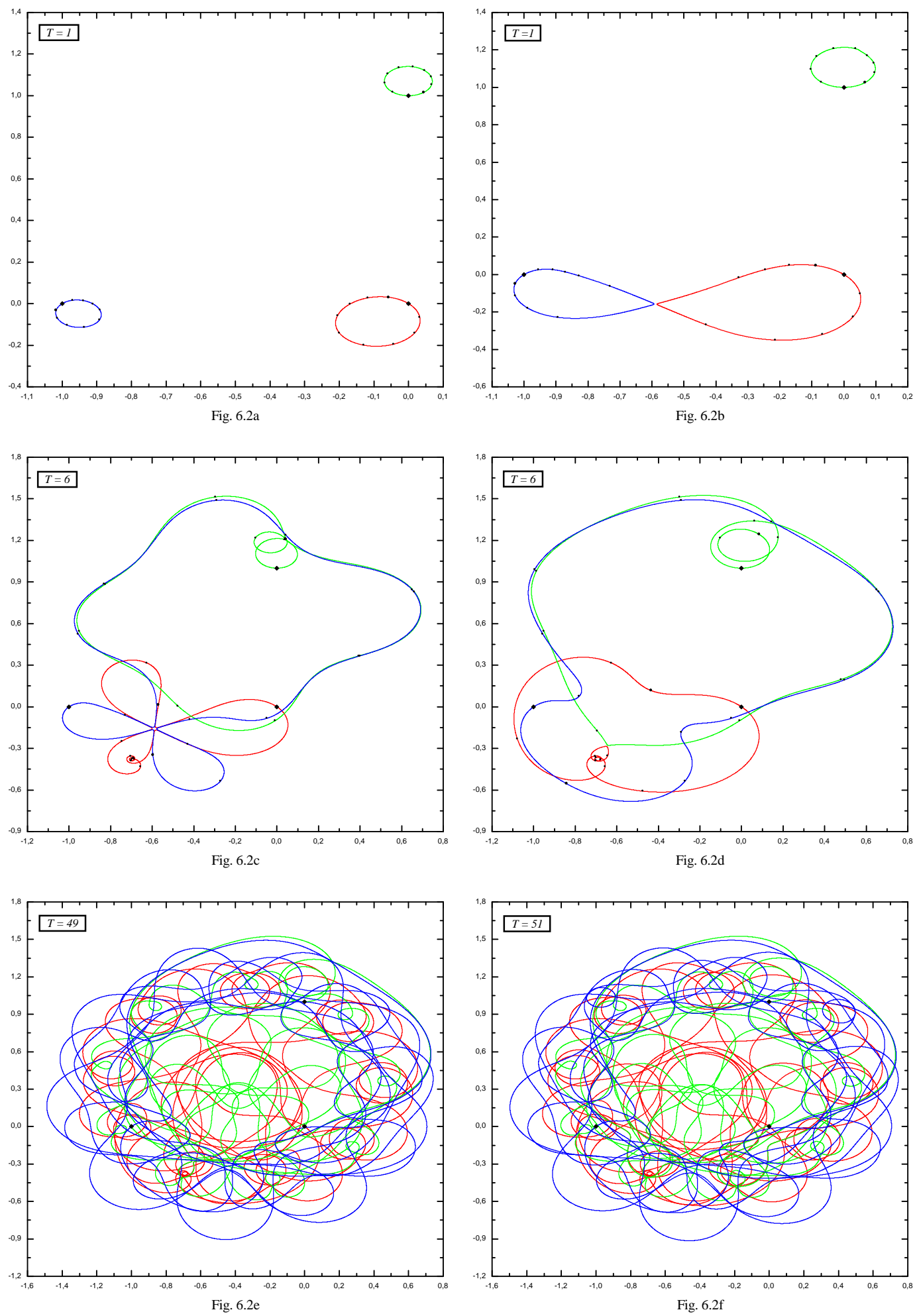

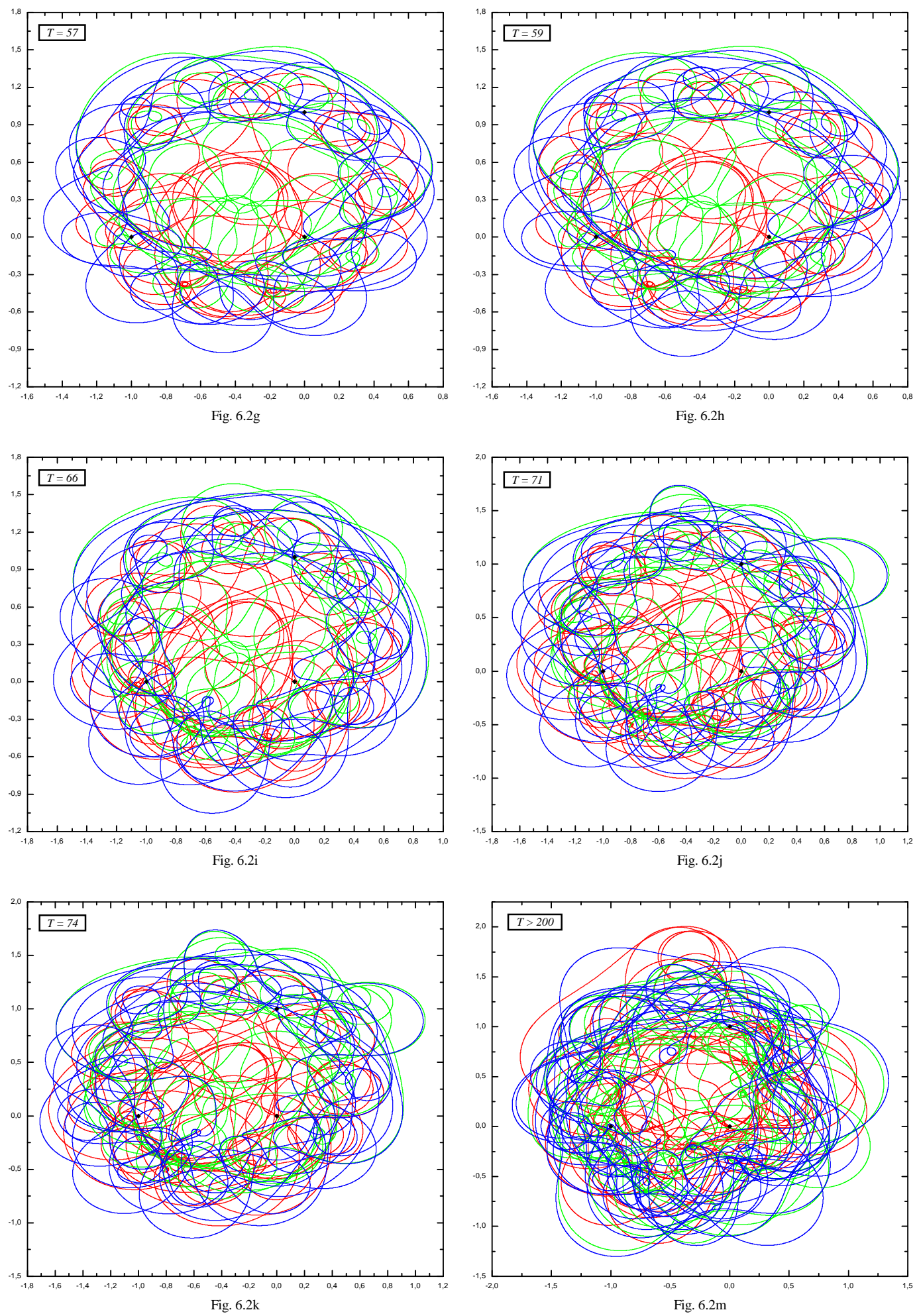
F Calogero, J-P Françoise and M Sommacal
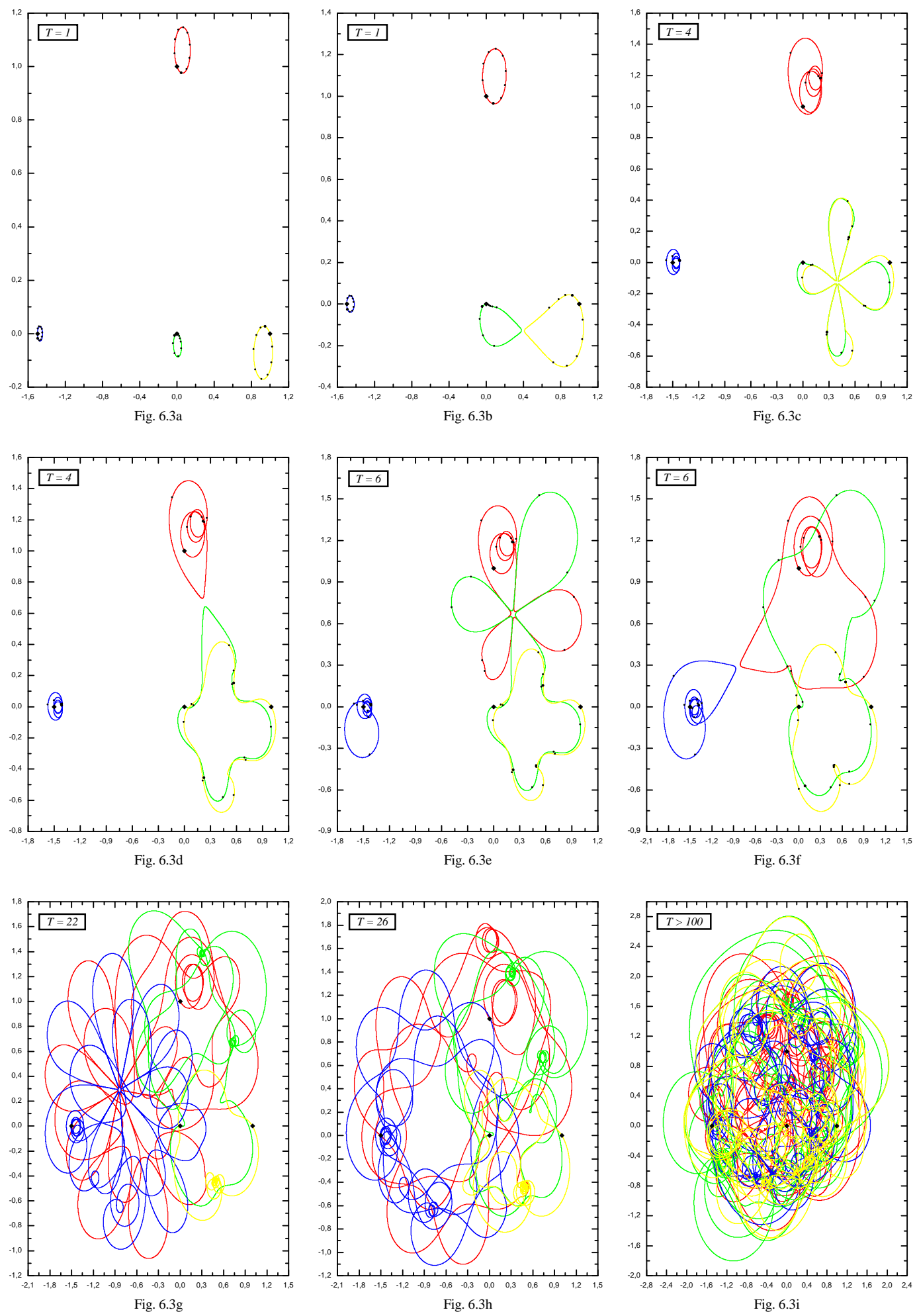

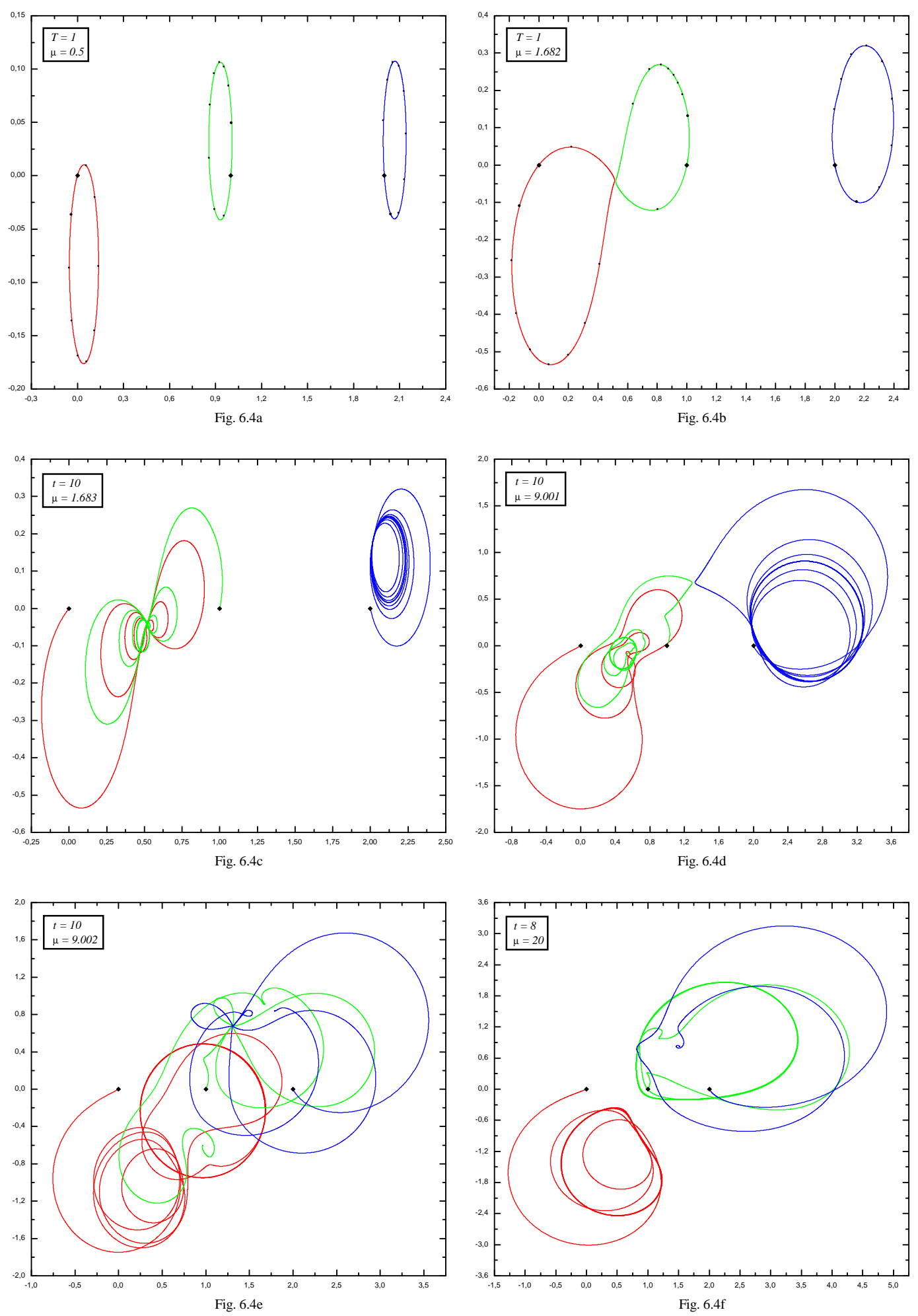

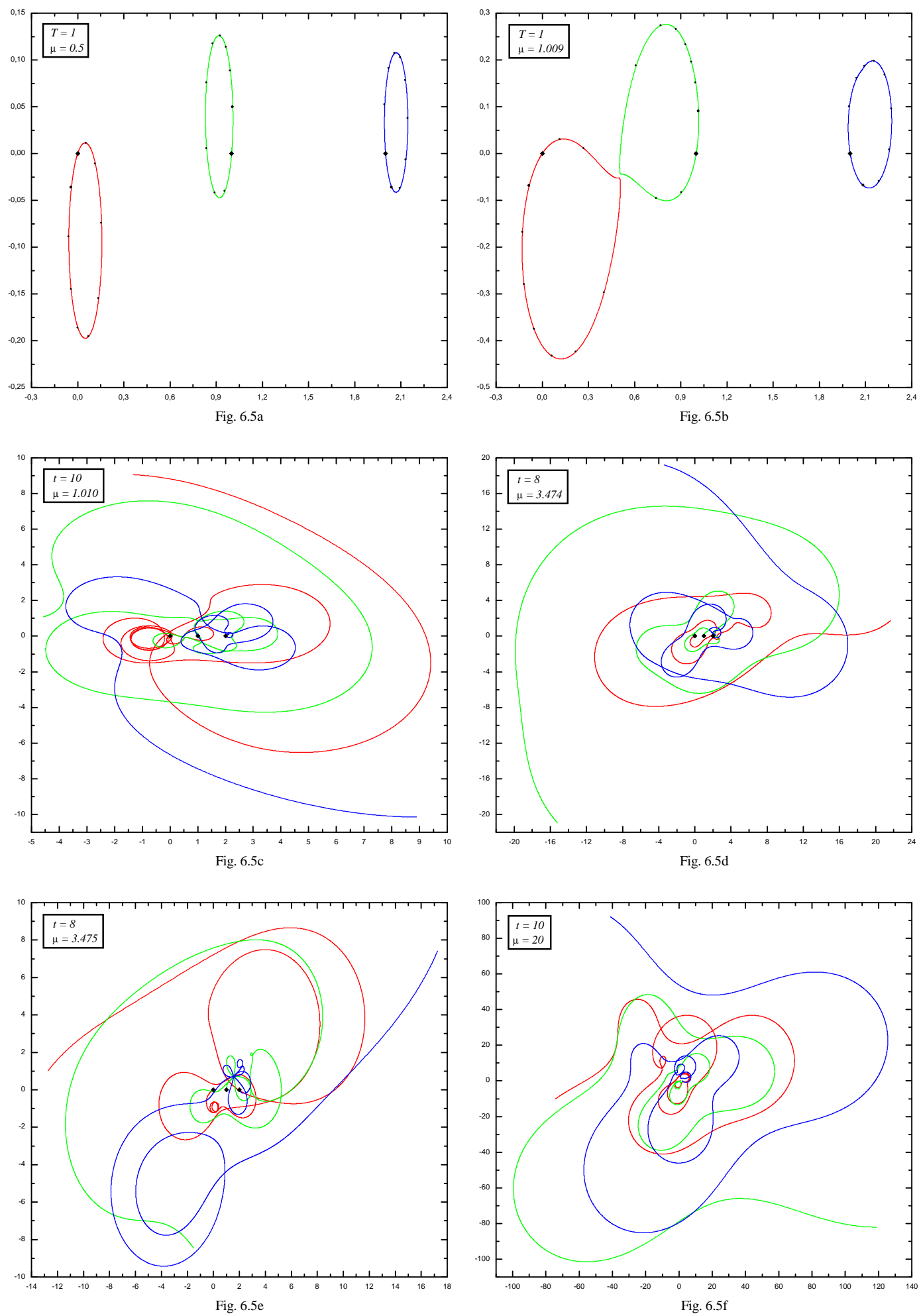


\section{Outlook}

The rich phenomenology featured by the solutions of the many-body problem in the plane investigated in this paper led us to refer to it, at least colloquially among us, as a "goldfish", thereby extending to this model the terminology that was initially suggested for its integrable variant [12]. Much additional fun and insight can certainly be gleaned from a more complete exploration of its solutions than we were able to report here; let us re-emphasize that the most illuminating way to pursue this study shall be provided by the computer code that simulates - by solving numerically the relevant equations of motions, see (1.1) - the actual behavior of the many-body problem as it unfolds over time; this computer code is indeed a remarkably efficient research tool which we hope to make available soon to the scientific community at large [8]. As for the more theoretical aspect of the investigation of this many-body problem, let us recall that in this paper a rather satisfactory understanding of the behavior of the system has been provided for the case in which the coupling constants $a_{n m}$ are all nonnegative, $a_{n m} \geq 0$, see (1.1) (or, equivalently, $\operatorname{Re}\left(a_{n m}\right) \geq 0$, see $\left.(1.5)\right)$, while the case when this restriction does not hold still needs to be fully investigated.

\section{Acknowledgements}

While the results reported in this paper were under development we discussed them with several colleagues, who often provided precious suggestions. In particular we like to thank for these Robert Conte, Ovidiu Costin, Herman Flaschka, Giovanni Gallavotti, Nalini Joshi, Martin Kruskal, François Leyvraz, Alexander Turbiner.

\section{Appendix A}

In this Appendix we provide a derivation of (1.9). Our starting point is the standard theorem that guarantees the analyticity of the solutions of (systems of) analytic ODEs (see for instance Section 13.21 of [7]). Our treatment is closely analogous to that of [10].

The standard formulation of this theorem refers to a system of first-order ODEs, say

$$
w_{j}^{\prime}=f_{j}\left(w_{1}, w_{2}, \ldots, w_{2 N}\right), \quad j=1,2, \ldots, 2 N,
$$

which coincides with our system, see (1.7), via the assignments

$$
\begin{aligned}
& w_{n} \equiv w_{n}(\tau)=\zeta_{n}(\tau)-\zeta_{n}(0), \\
& w_{N+n} \equiv w_{N+n}(\tau)=c\left[\zeta_{n}^{\prime}(\tau)-\zeta_{n}^{\prime}(0)\right],
\end{aligned}
$$

as well as

$$
\begin{aligned}
& f_{n}=c^{-1} w_{N+n}+\zeta_{n}^{\prime}(0) \\
& f_{N+n}=2 c \sum_{m=1, m \neq n}^{N} a_{n m} \frac{\left[c^{-1} w_{N+n}+\zeta_{n}^{\prime}(0)\right]\left[c^{-1} w_{N+m}+\zeta_{m}^{\prime}(0)\right]}{\left[w_{n}-w_{m}+\zeta_{n}(0)-\zeta_{m}(0)\right]} .
\end{aligned}
$$

Here of course (as in the rest of our paper) the index $n$ takes all integer values in the range $1 \leq n \leq N$, and $N$ is the total number of particles. Note that with these assignments 
all the $2 N$ quantities $w_{j}(\tau)$ vanish at the origin, $w_{j}(0)=0$, as appropriate to reproduce the notation of [7]. In these formulas $c$ is an arbitrary positive constant (which we have introduced for convenience, see below).

The standard result [7] provides then the following lower bound for the radius $\rho$ of the circular disk $D$, centered at the origin $\tau=0$ in the complex $\tau$-plane, within which the solutions $w_{n}(\tau)$ of (A.1), hence (see (A.2a)) as well the solutions $\zeta_{n}(\tau)$ of (1.7), are holomorphic:

$$
\rho>b /[(2 N+1) M]
$$

(this formula coincides with the last equation of Section 13.21 of [7], with the assignments $m=2 N$ and $a=\infty$, the first of which is justified by the fact that (A.1) features $2 N$ equations, the second of which is justified by the autonomous character of our equations of motion, see (A.1) with (A.3)). The two positive quantities $b$ and $M$ in this equation, (A.4), are defined as follows. The quantity $b$ is required to guarantee that the right-hand sides of (A.1) are holomorphic provided there hold the $2 N$ inequalities

$$
\left|w_{j}\right| \leq b
$$

this entails, in our case, the single restriction

$$
b<\tilde{\zeta} / 2
$$

with $\tilde{\zeta}$ defined by $(1.9 \mathrm{~b})$, because the only source of singularities is in our case the vanishing of a denominator in the right-hand side of (A.3b) (which is clearly excluded by (A.5) with (A.6) and (1.9b)). The second quantity in (A.4), $M \equiv M(b)$, is the upper bound of the right-hand sides of (A.1) when the quantities $w_{j}$ satisfy the restriction (A.5) with (A.6); but of course the inequality (A.4) holds a fortiori if we overestimate $M$ (as we shall now do).

Indeed, the right-hand sides of (A.3) clearly entail, via (A.2) and (1.9b,c),

$$
M<\max \left[c^{-1} b+\tilde{\zeta}^{\prime}, 2 c N \alpha\left(c^{-1} b+\tilde{\zeta}^{\prime}\right)^{2} /(\tilde{\zeta}-2 b)\right]
$$

where the maximum should be taken among the two terms, while it remains our privilege to choose the values of the positive quantity $c$, as well as the value of the positive quantity $b$ (respecting of course the inequality (A.6)). The positive quantity $\alpha$ in this formula is of course the maximal value of the moduli of the "coupling constants" $a_{n m}$,

$$
\alpha=\max _{n, m=1, \ldots, N ; n \neq m}\left|a_{n m}\right| .
$$

We now make the convenient choice

$$
c=[\tilde{\zeta}-2 b(1+N \alpha)] /\left[2 N \alpha \tilde{\zeta}^{\prime}\right]
$$

which equalizes the two terms inside the maximum function in (A.7). Note that the requirement that $c$ be positive entails that (A.6) must now be replaced by the more stringent inequality

$$
b<\tilde{\zeta} /[2(1+N \alpha)] .
$$


Hence from (A.7) we now get

$$
M<\tilde{\zeta}^{\prime}(\tilde{\zeta}-2 b) /[\tilde{\zeta}-2 b(1+N \alpha)]
$$

and via (A.4) there thus obtains the formula

$$
\rho>R \tilde{\zeta} / \tilde{\zeta}^{\prime}
$$

with

$$
R=x[1-2(1+N \alpha) x] /[(2 N+1)(1-2 x)],
$$

where the choice of the positive quantity $x=b / \tilde{\zeta}$ remains our privilege, provided it falls in the interval

$$
0<x<[2(1+N \alpha)]^{-1}
$$

which corresponds to (A.10).

Since (A.12) coincides with (1.9a), this latter equation is now proven. It would be easy to obtain the optimal choice of the constant $R$ by maximizing the right-hand side of (A.13) in the interval (A.14). More simply we just take $x$ in the middle of that interval (A.14), namely we set

$$
x=[4(1+N \alpha)]^{-1},
$$

and we thereby obtain for $R$ the following simple expression:

$$
R=[4(1+2 N)(1+2 N \alpha)]^{-1}
$$

\section{Appendix B}

In this appendix we investigate the nature of the branch points of the solutions of the first order ODE (4.4b),

$$
\left(\zeta^{\prime}\right)^{2}=V^{2}\left[1+(\zeta / L)^{-2 a}\right]
$$

with $V$ and $L$ arbitrary (nonvanishing) constants, obviously related to the initial data:

$$
V=\zeta_{1}^{\prime}(0)+\zeta_{2}^{\prime}(0)
$$

(see $(4.2))$, and

$$
L=\zeta(0)\left\{\left[\zeta^{\prime}(0) / V\right]^{2}-1\right\}^{1 /(2 a)}
$$

(see (B.1a)).

Let us begin by setting

$$
\zeta(\tau)=L f(w), \quad w=(V / L) \tau
$$


so that the equation satisfied by $f(w)$ read

$$
\left[f^{\prime}(w)\right]^{2}=1+[f(w)]^{-2 a}=1+[f(w)]^{\beta} .
$$

Here and below

$$
\beta=-2 a,
$$

and the prime appended to the function $f$ signifies of course differentiation with respect to its argument $w$.

Two possible mechanisms are at play to yield a singularity of $f(w)$, say at $w=w_{b}$ : (i) the vanishing of $f(w)$,

$$
f\left(w_{b}\right)=0 ;
$$

(ii) the divergence of $f(w)$,

$$
f\left(w_{b}\right)=\infty .
$$

Let us discuss them separately. But in both cases our approach will be based on replacing (B.3a) by a recursive set of equations, namely

$$
\left[{ }^{(j+1)} f^{\prime}(w)\right]^{2}=1+\left[{ }^{(j)} f(w)\right]^{-2 a}=1+\left[{ }^{(j)} f(w)\right]^{\beta}, \quad j=0,1,2, \ldots,
$$

and by assigning appropriately the initial element, ${ }^{(0)} f(w)$, of this iteration, so that it account properly for the leading behavior of the solution $f(w)$ in the neighborhood of the singular point $w=w_{b}$ - as demonstrated by the preservation of this singular behavior through the iteration (see below).

Let us begin with mechanism (i), by therefore setting

$$
{ }^{(j)} f\left(w_{b}\right)=0, \quad j=0,1,2, \ldots
$$

Firstly we consider the case characterized by the inequality

$$
\operatorname{Re}(a)>0,
$$

and we introduce correspondingly the exponent (see below)

$$
\gamma=1 /(1+a)
$$

which clearly then satisfies the restriction

$$
0<\operatorname{Re}(\gamma)<1
$$

Correspondingly we set (consistently with (B.6))

$$
{ }^{(0)} f(w)=\gamma^{-\gamma}\left(w-w_{b}\right)^{\gamma},
$$

and via (B.5) we then get

$$
\left[{ }^{(1)} f^{\prime}(w)\right]^{2}=\left[{ }^{(0)} f(w)\right]^{-2 a}\left\{1+\left[{ }^{(0)} f(w)\right]^{-2 a}\right\},
$$


namely

$$
{ }^{(1)} f^{\prime}(w)=\left[{ }^{(0)} f(w)\right]^{-a}\left\{1+\left[{ }^{(0)} f(w)\right]^{-2 a}\right\}^{1 / 2},
$$

namely (via (B.9))

$$
{ }^{(1)} f^{\prime}(w)=\gamma^{1-\gamma}\left(w-w_{b}\right)^{\gamma-1}\left\{1+\gamma^{2(\gamma-1)}\left(w-w_{b}\right)^{2(1-\gamma)}\right\}^{1 / 2} .
$$

To get this expression we used the relation

$$
a \gamma=1-\gamma
$$

which is clearly entailed by (B.8a).

In the neighborhood of $w=w_{b}$ we can rewrite (B.10c) as follows:

$$
\begin{aligned}
&(1) f^{\prime}(w)=\gamma^{1-\gamma}\left(w-w_{b}\right)^{\gamma-1} \\
& \quad \times\left\{1+\sum_{l=1}^{\infty}\left(\begin{array}{c}
1 / 2 \\
l
\end{array}\right) \gamma^{2 l(\gamma-1)}\left(w-w_{b}\right)^{2 l(1-\gamma)}\right\} .
\end{aligned}
$$

Here and below we use of course the usual notation for the binomial coefficient, so that

$$
\begin{aligned}
\left(\begin{array}{c}
1 / 2 \\
l
\end{array}\right) & =\Gamma(3 / 2)[\Gamma(3 / 2-l) \Gamma(1+l)]^{-1} \\
& =(-2)^{-(1+l)}(l-1 / 2)(2 l-1) ! ! / l ! .
\end{aligned}
$$

We then integrate (B.10d) (using (B.6) and (B.7b)) and we thereby get (using (B.8a))

$$
\begin{aligned}
{ }^{(1)} f(w)= & \gamma^{-\gamma}\left(w-w_{b}\right)^{\gamma} \\
& \times\left\{1+\sum_{l=1}^{\infty}(1+2 l a)^{-1}\left(\begin{array}{c}
1 / 2 \\
l
\end{array}\right) \gamma^{2 l(\gamma-1)}\left(w-w_{b}\right)^{2 l(1-\gamma)}\right\} .
\end{aligned}
$$

A comparison of this expression, (B.12), with (B.9) demonstrates the preservation of the leading term characterizing the behavior in the neighborhood of the singularity.

This result, as well as the following development, suggest setting

$$
\begin{gathered}
{ }^{(j)} f(w)=\gamma^{-\gamma}\left(w-w_{b}\right)^{\gamma}\left\{1+\sum_{l=1}^{\infty} \sum_{k=0}^{l}{ }^{(j)} g_{k l}\left(w-w_{b}\right)^{k \gamma}\left(w-w_{b}\right)^{2 l(1-\gamma)}\right\}, \\
j=0,1,2 \ldots,
\end{gathered}
$$

of course with

$$
{ }^{(0)} g_{k l}=0, \quad{ }^{(1)} g_{k l}=\delta_{k 0}(1+2 l a)^{-1}\left(\begin{array}{c}
1 / 2 \\
l
\end{array}\right) \gamma^{2 l(\gamma-1)} .
$$


We now show that this ansatz, (B.13), is preserved by the iteration (B.5), which we now rewrite in the following form (analogous to (B.10b)):

$$
{ }^{(j+1)} f^{\prime}(w)=\left[{ }^{(j)} f(w)\right]^{-a}\left\{1+\left[{ }^{(j)} f(w)\right]^{-2 a}\right\}^{1 / 2}
$$

Indeed the insertion of (B.15) in (B.13) yields

$$
\begin{gathered}
{ }^{(j+1)} f(w)=\gamma^{-\gamma}\left(w-w_{b}\right)^{\gamma}\left\{1+\sum_{l=1}^{\infty} \sum_{k=0}^{l}(j) \tilde{g}_{k l}\left(w-w_{b}\right)^{k \gamma}\left(w-w_{b}\right)^{2 l(1-\gamma)}\right\}, \\
j=0,1,2, \ldots,
\end{gathered}
$$

with the coefficients ${ }^{(j)} \tilde{g}_{k l}$ related to the coefficients ${ }^{(j)} g_{k l}$ by the formula

$$
\begin{aligned}
& \left\{1+\sum_{l=1}^{\infty} \sum_{k=0}^{l}{ }^{(j)} g_{k l} x^{k \gamma} y^{l}\right\}^{-a}\left[1+\gamma^{2(\gamma-1)} y\left\{1+\sum_{l=1}^{\infty} \sum_{k=0}^{l}{ }^{(j)} g_{k l} x^{k \gamma} y^{l}\right\}^{2 a}\right]^{1 / 2} \\
& =1+\sum_{l=1}^{\infty} \sum_{k=0}^{l}{ }^{(j)} \tilde{g}_{k l} x^{k \gamma} y^{l} .
\end{aligned}
$$

To get this formula, (B.16) with (B.17), we used again (B.8b) and we also set $x=\left(w-w_{b}\right)^{\gamma}$, $y=\left(w-w_{b}\right)^{2(1-\gamma)}$. A closed form expression of the coefficients ${ }^{(j)} \tilde{g}_{k l}$ in terms of the coefficients ${ }^{(j)} g_{k l}$ is not available, but there is no difficulty in principle to compute them sequentially, by expanding the left-hand side of (B.17) in powers of $x$ and $y$.

It is then clear that (B.16) can be integrated to yield (via (B.6) and (B.7b))

$$
\begin{aligned}
{ }^{(j+1)} f(w)= & \gamma^{-\gamma}\left(w-w_{b}\right)^{\gamma} \\
& \times\left\{1+\sum_{l=1}^{\infty} \sum_{k=0}^{l}{ }^{(j+1)} g_{k l}\left(w-w_{b}\right)^{k \gamma}\left(w-w_{b}\right)^{2 l(1-\gamma)}\right\},
\end{aligned}
$$

with

$$
{ }^{(j+1)} g_{k l}=(1+k+2 l a)^{-1(j)} \tilde{g}_{k l} .
$$

The consistency of (B.18) with (B.13) validates the ansatz (B.13) for all (nonnegative integer) values of the index $j$, and by assuming that this continues to hold in the limit as this index diverges, $j \rightarrow \infty$, and that in this limit ${ }^{(j)} f(w)$ yields the solution $f(w)$ of (B.3a), as indicated by a comparison of (B.3a) with (B.5), we infer for $f(w)$ the expression

$$
\begin{gathered}
f(w)=\gamma^{-\gamma}\left(w-w_{b}\right)^{\gamma}\left\{1+\sum_{l=1}^{\infty} \sum_{k=0}^{l} g_{k l}\left(w-w_{b}\right)^{k \gamma}\left(w-w_{b}\right)^{2 l(1-\gamma)}\right\}, \\
j=0,1,2, \ldots,
\end{gathered}
$$

Here the coefficients $g_{k l}$ are of course defined in terms of the above iteration, $g_{k l}={ }^{(\infty)} g_{k l}$, and they depend only on the "coupling constant" $a$ (and of course, as indicated by the 
notation, on the two indices $k$ and $l$ ), and we of course assume that, for $w$ sufficiently close to $w_{b}$, namely for $\left|w-w_{b}\right|$ sufficiently small, the infinite sum over the index $l$ in the right-hand side of this expression, (B.20), does converge.

Of course we could have directly started from the ansatz (B.20) and then verified its consistency with the nonlinear ODE (B.3a) - but the iterative approach appeared to us preferable in order to explain here how one arrives at such an ansatz.

Let us now proceed and discuss, always in the context of mechanism (i), the complementary case to (B.7a), namely let us now assume that

$$
\operatorname{Re}(a)<0,
$$

entailing of course

$$
\operatorname{Re}(\beta)>0 \text {. }
$$

It is now convenient to rewrite the iteration formula (B.5) in the form

$$
{ }^{(j+1)} f^{\prime}(w)= \pm\left\{1+\left[{ }^{(j)} f(w)\right]^{\beta}\right\}^{1 / 2}, \quad j=0,1,2, \ldots,
$$

and to solve it - consistently with (B.4a), again with the initial conditions (B.6)- by starting from the zeroth-order assignment

${ }^{(0)} f(w)= \pm\left(w-w_{b}\right)$.

We then get

$$
\begin{aligned}
& { }^{(1)} f^{\prime}(w)= \pm\left\{1+\left[ \pm\left(w-w_{b}\right)\right]^{\beta}\right\}^{1 / 2}, \\
& { }^{(1)} f^{\prime}(w)= \pm\left\{1+\sum_{l=1}^{\infty}\left(\begin{array}{c}
1 / 2 \\
l
\end{array}\right)\left[ \pm\left(w-w_{b}\right)\right]^{l \beta}\right\}
\end{aligned}
$$

entailing, via (B.6),

$$
{ }^{(1)} f(w)= \pm\left(w-w_{b}\right)\left\{1+\sum_{l=1}^{\infty}(1+l \beta)^{-1}\left(\begin{array}{c}
1 / 2 \\
l
\end{array}\right)\left[ \pm\left(w-w_{b}\right)\right]^{l \beta}\right\} .
$$

We now set, as suggested by this formula, (B.25),

$$
{ }^{(j)} f(w)= \pm\left(w-w_{b}\right)\left\{1+\sum_{l=1}^{\infty}{ }^{(j)} g_{l}\left[ \pm\left(w-w_{b}\right)\right]^{l \beta}\right\}, \quad j=0,1,2, \ldots,
$$

and verify the consistency of this ansatz, (B.26), with the iteration (B.22). Indeed, by proceeding as above, we get

$$
{ }^{(j+1)} f(w)= \pm\left(w-w_{b}\right)\left\{1+\sum_{l=1}^{\infty}{ }^{(j+1)} g_{l}\left[ \pm\left(w-w_{b}\right)\right]^{l \beta}\right\}
$$


with

$$
{ }^{(j+1)} g_{l}=(1+l \beta)^{-1(j)} \tilde{g}_{l}
$$

and

$$
\left\{1+x\left[1+\sum_{l=1}^{\infty}{ }^{(j)} g_{l} x^{l}\right]\right\}^{1 / 2}=1+\sum_{l=1}^{\infty}{ }^{(j)} \tilde{g}_{l} x^{l} .
$$

So, in analogy to the preceding case, we infer now the following expression for the solution of (B.3a) with (B.4a) and (B.21):

$$
f(w)= \pm\left(w-w_{b}\right)\left\{1+\sum_{l=1}^{\infty} g_{l}\left[ \pm\left(w-w_{b}\right)\right]^{l \beta}\right\} .
$$

The significance of the choice among one or the other alternative for the \pm sign is explained in Section 4.

Let us now turn to the consideration of mechanism (ii), see (B.4b). In this case the assumption (B.7a) is inconsistent with (B.4b), because (B.4b) with (B.7a) entails, via (B.3a), that $f^{\prime}\left(w_{b}\right)= \pm 1$, while a finite value of $f^{\prime}\left(w_{b}\right)$ is clearly inconsistent with (B.4b). It is moreover clear that the assumption (B.7a) is consistent with (B.21) only if this inequality is strengthened to read

$$
\operatorname{Re}(a)<-1
$$

entailing (see (B.8a))

$$
\operatorname{Re}(\gamma)<0
$$

Then the appropriate representation of the solution is given by (B.20), which is then clearly consistent with (B.4b) due to (B.31b) (and note that all the terms in the sums in the right-hand side of this representation, (B.20), become vanishingly small for $w \approx w_{b}$, again thanks to (B.31b), because the index $k$ is limited not to exceed the index $l$ ).

This concludes our analysis, which validates the results reported in Section 4.

Let us end this Appendix with a final remark, based on (B.1), which we conveniently rewrite in the following form (see (4.4a)):

$$
\left(\zeta^{\prime}\right)^{2}-B \zeta^{-2 a}=V^{2}
$$

where of course $V$ is given in terms of the initial data by (B.1b) and $B$ by the formula (see (B.1b) and (4.1b))

$$
B=-4 \zeta_{1}^{\prime}(0) \zeta_{2}^{\prime}(0)\left[\zeta_{1}(0)-\zeta_{2}(0)\right]^{2 a} .
$$

Assume now that the coupling constant $a$ is a negative integer, $a=-p$, so that (B.32) reads

$$
\left(\zeta^{\prime}\right)^{2}+4 \zeta_{1}^{\prime}(0) \zeta_{2}^{\prime}(0)\left[\zeta_{1}(0)-\zeta_{2}(0)\right]^{-2 p} \zeta^{2 p}=V^{2}
$$


and assume moreover that the initial data, $\zeta_{1}(0), \zeta_{2}(0), \zeta_{1}^{\prime}(0), \zeta_{2}^{\prime}(0)$, are all real, so that this equation of motion, (B.33), and its solution, are also real (for real $\tau$ ), and the solution of the equations of motion (1.7) is as well real for real $\tau$. It is then clear from (B.33) (which can then be interpreted as the energy conservation formula for a one-dimensional particle in a power-law potential which vanishes at the origin and diverges at large distance) that, if the two initial "velocities" $\zeta_{1}^{\prime}(0), \zeta_{2}^{\prime}(0)$ have the same sign, so that the potential energy (i.e., the second term in the left-hand side of (B.33)) diverges at large distances to positive infinity, then the solution $\zeta(\tau)$ of this equation (B.33) is, for real $\tau$, always periodic, albeit with a period which depends on the initial data. In this case, however, the motion of the two "particles" $\zeta_{1}(\tau)$ and $\zeta_{2}(\tau)$ will not be periodic, due to the drift associated with the uniform motion of their center of mass (note that, if the two initial "velocities" $\zeta_{1}^{\prime}(0)$, $\zeta_{2}^{\prime}(0)$ have the same sign, the center of mass will indeed move). Let us emphasize that this discussion refers to the solution of the equations of motion (1.7) in the special case in which they can themselves be interpreted to represent the (one-dimensional) motion of real particles. For explicit examples of this phenomenon see the treatments in Section 4 of the two-body problems with pair coupling constants $a=-1, a=-2$ and $a=-3$, respectively.

\section{Appendix C}

In this Appendix we outline the treatment that justifies the main results of Section 5 . Hence we investigate the behavior of the (generic) solution $\zeta(\tau)$ of $(1.7)$ in the neighborhood of its singularities. In analogy to what we did in Appendix B our basic approach is to replace (1.7) with the following recursive set of equations

$$
{ }^{(j+1)} \zeta_{n}^{\prime \prime}=2 \sum_{m=1 ; m \neq n}^{N} a_{n m}{ }^{(j)} \zeta_{n}^{\prime(j)} \zeta_{m}^{\prime} /\left({ }^{(j)} \zeta_{n}-{ }^{(j)} \zeta_{m}\right), \quad j=0,1,2, \ldots
$$

and to solve this recursive sequence of equations by starting from an appropriate assignment for the zeroth-order term ${ }^{(0)} \zeta(\tau)$ so that it capture the leading behavior of the solution $\underline{\zeta}(\tau)$ in the neighborhood of the singularity whose nature we wish to ascertain. Let us emphasize that this procedure is only supposed to work in the neighborhood of the value, $\tau=\tau_{b}$, at which a singularity occurs, and its effectiveness is predicated upon the successful identification of the zeroth-order assignment ${ }^{(0)} \underline{\zeta}(\tau)$, as demonstrated by the subsequent preservation of the leading part of its behavior for $\tau \approx \tau_{b}$ throughout the iteration (see examples below). We restrict our treatment to a demonstration of this fact; to qualify as a complete proof our analysis should be complemented by two additional, synergistically related, results (which will instead be taken for granted here): (i) a proof that the resulting representation of $\underline{\zeta}(\tau)$ as an infinite series (see for instance (5.4), (5.6) and (5.9)) does converge in a sufficiently small neighborhood of the singular point $\tau=\tau_{b}$, and (ii) a proof that the sequence produced by the iteration (C.1) (supplemented by appropriate "initial conditions" at every iteration cycle: see examples below) does converge to a solution $\underline{\zeta}(\tau)$ of $(1.7)$,

$$
\lim _{j \rightarrow \infty}\left[{ }^{(j)} \underline{\zeta}\right]=\underline{\zeta}(\tau)
$$


Throughout this Appendix $\mathrm{C}$ we use the notation of Section 5 without reporting anew the corresponding definitions. Our first task is to justify (5.4). To this end we set

$$
\begin{aligned}
& { }^{(0)} \zeta_{1}(\tau)=b+c\left(\tau-\tau_{b}\right)^{\gamma}+v\left(\tau-\tau_{b}\right), \\
& { }^{(0)} \zeta_{2}(\tau)=b-c\left(\tau-\tau_{b}\right)^{\gamma}+v\left(\tau-\tau_{b}\right), \\
& { }^{(0)} \zeta_{n}(\tau)=b_{n}+v_{n}\left(\tau-\tau_{b}\right), \quad n=3, \ldots, N .
\end{aligned}
$$

From this assignment and (C.1) (with $j=0$ and $n=1$ ) we get

$$
\begin{aligned}
& { }^{(1)} \zeta_{1}^{\prime \prime}=2 a\left[c \gamma\left(\tau-\tau_{b}\right)^{\gamma-1}+v\right]\left[-c \gamma\left(\tau-\tau_{b}\right)^{\gamma-1}+v\right] /\left[2 c\left(\tau-\tau_{b}\right)^{\gamma}\right] \\
& +2 \sum_{m=3}^{N} a_{1 m}\left[c \gamma\left(\tau-\tau_{b}\right)^{\gamma-1}+v\right] v_{m} /\left[b-b_{m}+c\left(\tau-\tau_{b}\right)^{\gamma}+\left(v-v_{m}\right)\left(\tau-\tau_{b}\right)\right] \\
& =-a \gamma^{2} c\left(\tau-\tau_{b}\right)^{\gamma-2}\left[1-v^{2}(c \gamma)^{-2}\left(\tau-\tau_{b}\right)^{2-2 \gamma}\right] \\
& +2 c \gamma\left(\tau-\tau_{b}\right)^{\gamma-1}\left[1+v(c \gamma)^{-1}\left(\tau-\tau_{b}\right)^{1-\gamma}\right] \\
& \times \sum_{m=3}^{N} a_{1 m} v_{m}\left(b-b_{m}\right)^{-1}\left\{1+\left(b-b_{m}\right)^{-1}\left[c\left(\tau-\tau_{b}\right)^{\gamma}+\left(v-v_{m}\right)\left(\tau-\tau_{b}\right)\right]\right\}^{-1} .
\end{aligned}
$$

The motivation to re-write (C.4a) in the form (C.4b) is of course based on (5.2b), which is as well essential for the subsequent developments.

We now use the following formula:

$$
(1-\varepsilon-\delta)^{-1}=\sum_{k=0}^{\infty} \sum_{l=0}^{k}\left(\begin{array}{c}
k \\
l
\end{array}\right) \varepsilon^{k-l} \delta^{l},
$$

where

$$
\left(\begin{array}{c}
k \\
l
\end{array}\right)=k ! /[l !(k-l) !]
$$

is the binomial coefficient, which is hereafter understood to vanish if $l$ or $(k-l)$ is a negative integer. This formula, (C.5), is of course only applicable provided

$$
|\varepsilon+\delta|<1 .
$$

Via (C.5) we rewrite (C.4b) as follows:

$$
\begin{aligned}
{ }^{(1)} \zeta_{1}^{\prime \prime}= & \gamma(\gamma-1) c\left(\tau-\tau_{b}\right)^{\gamma-2}+\left(a v^{2} / c\right)\left(\tau-\tau_{b}\right)^{-\gamma} \\
& +\left[1+v(c \gamma)^{-1}\left(\tau-\tau_{b}\right)^{1-\gamma}\right] \sum_{k=0}^{\infty} \sum_{l=0}^{k} f_{k l}^{(1)}\left(\tau-\tau_{b}\right)^{(k-l+1) \gamma+l-1},
\end{aligned}
$$

where

$$
\begin{aligned}
f_{k l}^{(1)}= & 2 c \gamma\left(\begin{array}{c}
k \\
l
\end{array}\right) \sum_{m=3}^{N} a_{1 m} v_{m}\left(b-b_{m}\right)^{-1} \\
& \times\left[-c /\left(b-b_{m}\right)\right]^{k-l}\left[-\left(v-v_{m}\right) /\left(b-b_{m}\right)\right]^{l},
\end{aligned}
$$


and we also took advantage of the relation

$$
\gamma-1=-a \gamma
$$

entailed by the definition (5.2a) of $\gamma$. Note that the step from (C.4c) to (C.4d) is justified provided the following condition holds (which corresponds to (C.6a)):

$$
\left|c\left(\tau-\tau_{b}\right)^{\gamma}+\left(v-v_{m}\right)\left(\tau-\tau_{b}\right)\right|<\left|b-b_{m}\right|,
$$

which is certainly true (see $(5.2 \mathrm{~b})$ ) provided $\tau$ is sufficiently close to $\tau_{b}$, namely $\left|\tau-\tau_{b}\right|$ is sufficiently small.

We now integrate twice (C.4c), adjusting the two integration constants so that, for $\tau \approx \tau_{b}$, the three leading terms of ${ }^{(1)} \zeta_{1}(\tau)$ coincide with ${ }^{(0)} \zeta_{1}(\tau)$, see (C.3a) (note that the first correction term next to the constant one, namely the second one of the three, is automatically $\mathrm{OK}$ ) and we thereby get

$$
\begin{aligned}
{ }^{(1)} \zeta_{1}(\tau)= & b+c\left(\tau-\tau_{b}\right)^{\gamma}+v\left(\tau-\tau_{b}\right)+a v^{2}[c(2-\gamma)(1-\gamma)]^{-1}\left(\tau-\tau_{b}\right)^{2-\gamma} \\
& +\sum_{j=1}^{\infty} \sum_{k=1}^{\infty}{ }^{(1)} g_{j k}^{(1)}\left(\tau-\tau_{b}\right)^{j}\left(\tau-\tau_{b}\right)^{k \gamma}
\end{aligned}
$$

with

$$
\begin{aligned}
{ }^{(1)} g_{j k}^{(1)}= & (j+k \gamma)^{-1}(j-1+k \gamma)^{-1} f_{j+k-2, j-1}^{(1)} \\
& +v(c \gamma)^{-1}[j+1+(k-1) \gamma]^{-1}[j+(k-1) \gamma]^{-1} f_{j+k-2, j}^{(1)} .
\end{aligned}
$$

In a completely analogous manner we get

$$
\begin{aligned}
{ }^{(2)} \zeta_{1}(\tau)= & b-c\left(\tau-\tau_{b}\right)^{\gamma}+v\left(\tau-\tau_{b}\right)-a v^{2}[c(2-\gamma)(1-\gamma)]^{-1}\left(\tau-\tau_{b}\right)^{2-\gamma} \\
& +\sum_{j=1}^{\infty} \sum_{k=1}^{\infty}{ }^{(1)} g_{j k}^{(2)}\left(\tau-\tau_{b}\right)^{j}\left(\tau-\tau_{b}\right)^{k \gamma}
\end{aligned}
$$

with

$$
\begin{aligned}
{ }^{(1)} g_{j k}^{(2)}= & (j+k \gamma)^{-1}(j-1+k \gamma)^{-1} f_{j+k-2, j-1}^{(2)} \\
& -v(c \gamma)^{-1}[j+1+(k-1) \gamma]^{-1}[j+(k-1) \gamma]^{-1} f_{j+k-2, j}^{(2)},
\end{aligned}
$$

where

$$
\begin{aligned}
f_{k l}^{(2)}= & -2 c \gamma\left(\begin{array}{c}
k \\
l
\end{array}\right) \sum_{m=3}^{N} a_{2 m} v_{m}\left(b-b_{m}\right)^{-1} \\
& \times\left[c /\left(b-b_{m}\right)\right]^{k-l}\left[-\left(v-v_{m}\right) /\left(b-b_{m}\right)\right]^{l} .
\end{aligned}
$$

Also analogous is the derivation of ${ }^{(1)} \zeta_{n}(\tau), n=3, \ldots, N$ :

$$
\begin{aligned}
{ }^{(1)} \zeta_{n}(\tau)= & b_{n}+v_{n}\left(\tau-\tau_{b}\right) \\
& +\sum_{j=1}^{\infty} \sum_{k=\delta_{j 1}}^{\infty}{ }^{(1)} g_{j k}^{(n)}\left(\tau-\tau_{b}\right)^{j}\left(\tau-\tau_{b}\right)^{k \gamma}, \quad n=3, \ldots, N,
\end{aligned}
$$


with

$$
\begin{aligned}
{ }^{(1)} g_{j k}^{(n)}= & {[(j+k \gamma)(j-1+k \gamma)]^{-1}\left\{\left(1-\delta_{j 1}\right)\left[v_{n} /\left(b_{n}-b\right)\right]\left[c /\left(b_{n}-b\right)\right]^{k}\right.} \\
& \times\left[-\left(v_{n}-v\right) /\left(b_{n}-b\right)\right]^{j-2}\left[v+k(j-1)^{-1} \gamma\left(v-v_{n}\right)\right] \\
& \times\left(\begin{array}{c}
j+k-2 \\
k
\end{array}\right) 2 \sum_{s=1,2} a_{n s}(-1)^{(s-1) k} \\
& \left.+\delta_{k, 0} 2 \sum_{m=3}^{N} a_{n m} v_{n} v_{m}\left(b_{n}-b_{m}\right)^{-1}\left[-\left(v_{n}-v_{m}\right) /\left(b_{n}-b_{m}\right)\right]^{j-1}\right\} .
\end{aligned}
$$

At this point we introduce the ansatz

$$
\begin{aligned}
{ }^{(j)} \zeta_{s}(\tau)= & b+(-1)^{s-1} c\left(\tau-\tau_{b}\right)^{\gamma}+v\left(\tau-\tau_{b}\right) \\
& +\sum_{k=1}^{\infty} \sum_{l, m=0 ; l+m \geq 1}^{\infty}{ }^{(j)} g_{k l m}^{(s)}\left(\tau-\tau_{b}\right)^{k+l \gamma+m(1-\gamma)}, \quad s=1,2, \\
{ }^{(j)} \zeta_{n}(\tau)= & b_{n}+v_{n}\left(\tau-\tau_{b}\right) \\
& +\sum_{k=1}^{\infty} \sum_{l=\delta_{k 1}}^{\infty} \sum_{m=0}^{\infty}{ }^{(j)} g_{k l m}^{(n)}\left(\tau-\tau_{b}\right)^{k+l \gamma+m(1-\gamma)}, \quad n=3, \ldots, N,
\end{aligned}
$$

which is clearly consistent (for the appropriate choice of the coefficients $g_{k l m}^{(n)}$ ) with both (C.3) $(j=0)$ and $(\mathrm{C} .10,11)(j=1)$, and we show that it is moreover consistent with the iteration (C.1), namely that insertion of this ansatz, (C.12), in the right-hand side of (C.1) yields, after appropriate expansions and integrations, its validity at the iteration order $j+1$. To this end one must of course use the formal expansion formula

$$
\left\{1+\sum_{k=1}^{\infty} g_{k} \varepsilon^{k}\right\}^{-1}=1+\sum_{k=1}^{\infty} \tilde{g}_{k} \varepsilon^{k},
$$

where we assume that the coefficients $g_{k}$ are given and the coefficients $\tilde{g}_{k}$ are obtained from them (clearly this can be done sequentially starting from the lowest powers, although a closed form expression is not generally available). Of course this formula can only hold under the restriction

$$
|\varepsilon|<E,
$$

where the constant $E$ is required to be small enough so that all the sums in (C.13a) converge (and in order that this be possible appropriate restrictions must hold to begin with on the behavior of the coefficients $g_{k}$ when their indices diverge, to guarantee that the sum in the left-hand side of this formula, (C.13a), do converge when (C.13b) holds).

It is easy to convince oneself that all one in fact needs to prove is that the following expansions,

$$
\begin{aligned}
{ }^{(j)} \zeta_{s}(\tau)= & b+(-1)^{(s-1)} c\left(\tau-\tau_{b}\right)^{\gamma}+v\left(\tau-\tau_{b}\right) \\
& +O\left(\left|\left(\tau-\tau_{b}\right)\right|^{1+\gamma},\left|\left(\tau-\tau_{b}\right)\right|^{2-\gamma}\right), \quad s=1,2, \\
{ }^{(j)} \zeta_{n}(\tau)= & b_{n}+v_{n}\left(\tau-\tau_{b}\right)+O\left(\left|\left(\tau-\tau_{b}\right)\right|^{1+\gamma}\right), \quad n=3, \ldots, N,
\end{aligned}
$$


which are clearly entailed by (C.12) (and are of course valid for $\tau \approx \tau_{b}$ ), are consistent with the iteration (C.1), namely that their validity at the order $j$, see (C.14), entails their validity at the order $j+1$. Indeed this result (once proven) entails that the iteration process keeps producing only the powers of $\left(\tau-\tau_{b}\right)$ that appear in the right-hand side of (C.12) (and no logarithms!), with the coefficients ${ }^{(j+1)} g_{k l m}^{(n)}$ uniquely determined via (C.1) by the coefficients ${ }^{(j)} g_{k l m}^{(n)}$ and the other constants appearing in the right-hand side of (C.12).

To prove the consistency of (C.14) with (C.1) we now note that these formulas, (C.14), entail

$$
\begin{aligned}
{ }^{(j)} \zeta_{s}^{\prime}(\tau)= & \gamma(-1)^{(s-1)} c\left(\tau-\tau_{b}\right)^{\gamma-1}+v \\
& +O\left(\left.\left|\left(\tau-\tau_{b}\right)\right|^{\gamma}|,|\left(\tau-\tau_{b}\right)\right|^{1-\gamma}\right), \quad s=1,2, \\
{ }^{(j)} \zeta_{n}^{\prime}(\tau)= & v_{n}+O\left(\left|\left(\tau-\tau_{b}\right)\right|^{\gamma}\right), \quad n=3, \ldots, N,
\end{aligned}
$$

hence

$$
\begin{aligned}
{ }^{(j)} \zeta_{1}^{\prime}(\tau){ }^{(j)} \zeta_{2}^{\prime}(\tau)= & -\gamma^{2} c^{2}\left(\tau-\tau_{b}\right)^{2(\gamma-1)}+v^{2} \\
& +O\left(\left|\left(\tau-\tau_{b}\right)\right|^{2 \gamma-1},\left|\left(\tau-\tau_{b}\right)\right|\right), \\
{ }^{(j)} \zeta_{s}^{\prime}(\tau)^{(j)} \zeta_{n}^{\prime}(\tau)= & (-1)^{(s-1)} \gamma c v_{n}\left(\tau-\tau_{b}\right)^{\gamma-1}+v v_{n}+O\left(\left|\left(\tau-\tau_{b}\right)\right|^{\gamma}\left|\left(\tau-\tau_{b}\right)\right|^{1-\gamma}\right), \\
s=1,2, \quad n=3, \ldots, N, & \\
{ }^{(j)} \zeta_{n}^{\prime}(\tau)^{(j)} \zeta_{m}^{\prime}(\tau)= & v_{n} v_{m}+O\left(\left|\left(\tau-\tau_{b}\right)\right|^{\gamma}\right), \quad n, m=3, \ldots, N,
\end{aligned}
$$

and

$$
\begin{aligned}
& { }^{(j)} \zeta_{1}(\tau)-{ }^{(j)} \zeta_{2}(\tau)=2 c\left(\tau-\tau_{b}\right)^{\gamma}+O\left(\left|\left(\tau-\tau_{b}\right)\right|^{1+\gamma},\left|\left(\tau-\tau_{b}\right)\right|^{2-\gamma}\right), \\
& { }^{(j)} \zeta_{s}(\tau)-{ }^{(j)} \zeta_{n}(\tau)=b-b_{n}+O\left(\left|\left(\tau-\tau_{b}\right)\right|^{\gamma}\right), \quad s=1,2, \quad n=3, \ldots, N, \\
& { }^{(j)} \zeta_{n}(\tau)-{ }^{(j)} \zeta_{m}(\tau)=b_{n}-b_{m}+O\left(\left|\left(\tau-\tau_{b}\right)\right|^{1+\gamma}\right), \quad n, m=3, \ldots, N,
\end{aligned}
$$

hence

$$
\begin{aligned}
{\left[{ }^{(j)} \zeta_{1}(\tau)-{ }^{(j)} \zeta_{2}(\tau)\right]^{-1}=} & (2 c)^{-1}\left(\tau-\tau_{b}\right)^{-\gamma} \\
& \times\left[1+O\left(\left|\left(\tau-\tau_{b}\right)\right|,\left|\left(\tau-\tau_{b}\right)\right|^{2(1-\gamma)}\right)\right], \\
{\left[{ }^{(j)} \zeta_{s}(\tau)-{ }^{(j)} \zeta_{n}(\tau)\right]^{-1}=} & \left(b-b_{n}\right)^{-1}\left[1+O\left(\left|\left(\tau-\tau_{b}\right)\right|^{\gamma}\right)\right], \\
& s=1,2 ; \quad n=3, \ldots, N, \\
{\left[{ }^{(j)} \zeta_{n}(\tau)-{ }^{(j)} \zeta_{m}(\tau)\right]^{-1}=} & \left(b_{n}-b_{m}\right)^{-1}\left[1+O\left(\left|\left(\tau-\tau_{b}\right)\right|^{1+\gamma}\right)\right], \\
& n, m=3, \ldots, N .
\end{aligned}
$$


It is therefore clear that (C.1) entails (via (C.16a,b) and (C.17a,b))

$$
\begin{aligned}
(j+1) & \zeta_{s}^{\prime \prime}(\tau)=2 a\left[-\gamma^{2} c^{2}\left(\tau-\tau_{b}\right)^{2(\gamma-1)}+v^{2}+O\left(\left|\left(\tau-\tau_{b}\right)\right|^{2 \gamma-1},\left|\left(\tau-\tau_{b}\right)\right|\right)\right] \\
& \times(-1)^{s-1}(2 c)^{-1}\left(\tau-\tau_{b}\right)^{-\gamma}\left[1+O\left(\left|\left(\tau-\tau_{b}\right)\right|,\left|\left(\tau-\tau_{b}\right)\right|^{2(1-\gamma)}\right)\right] \\
& +2 \sum_{m=3}^{N} a_{s m}\left[(-1)^{(s-1)} \gamma c v_{n}\left(\tau-\tau_{b}\right)^{\gamma-1}+v v_{n}+O\left(\left|\left(\tau-\tau_{b}\right)\right|^{\gamma},\left|\left(\tau-\tau_{b}\right)\right|^{1-\gamma}\right)\right] \\
& \times\left(b-b_{n}\right)^{-1}\left[1+O\left(\left|\left(\tau-\tau_{b}\right)\right|^{\gamma}\right)\right], \quad s=1,2, \quad \text { (C.19a) }
\end{aligned}
$$

namely

$$
\begin{aligned}
{ }^{(j+1)} \zeta_{s}^{\prime \prime}(\tau)= & (-1)^{s-1} \gamma(\gamma-1)\left(\tau-\tau_{b}\right)^{\gamma-2} \\
& +O\left(\left|\left(\tau-\tau_{b}\right)\right|^{-\gamma},\left|\left(\tau-\tau_{b}\right)^{\gamma-1}\right|\right), \quad s=1,2
\end{aligned}
$$

Integration (with the appropriate choice of integration constants) of this equation, (C.19b), yields

$$
\begin{aligned}
{ }^{(j+1)} \zeta_{s}(\tau)= & b+(-1)^{(s-1)} c\left(\tau-\tau_{b}\right)^{\gamma}+v\left(\tau-\tau_{b}\right) \\
& +O\left(\left|\left(\tau-\tau_{b}\right)\right|^{1+\gamma},\left|\left(\tau-\tau_{b}\right)\right|^{2-\gamma}\right), \quad s=1,2,
\end{aligned}
$$

and the consistency of this expression, (C.20), with (C.14a) is plain.

It is likewise clear that (C.1) entails (via (C.16a,c) and (C.17a,c))

$$
\begin{aligned}
{ }^{(j+1)} \zeta_{n}^{\prime \prime}(\tau) & =2 \sum_{s=1,2}\left\{a _ { n s } \left[(-1)^{(s-1)} \gamma c v_{n}\left(\tau-\tau_{b}\right)^{\gamma-1}+v v_{n}\right.\right. \\
+ & \left.\left.O\left(\left|\left(\tau-\tau_{b}\right)\right|^{\gamma},\left|\left(\tau-\tau_{b}\right)\right|^{1-\gamma},\left|\left(\tau-\tau_{b}\right)\right|^{2 \gamma-1}\right)\right]\left(b_{n}-b\right)^{-1}\left[1+O\left(\left|\left(\tau-\tau_{b}\right)\right|^{\gamma}\right)\right]\right\} \\
+ & 2 \sum_{m=3}^{N}\left\{a_{n m}\left[v_{n} v_{m}+O\left(\left|\left(\tau-\tau_{b}\right)\right|^{\gamma}\right)\right]\left(b_{n}-b_{m}\right)^{-1}\left[1+O\left(\left|\left(\tau-\tau_{b}\right)\right|^{1+\gamma}\right)\right]\right\} \\
n & =3, \ldots, N,
\end{aligned}
$$

namely

$$
{ }^{(j+1)} \zeta_{n}^{\prime \prime}(\tau)=O\left(\left|\tau-\tau_{b}\right|^{\gamma-1}\right), \quad n=3, \ldots, N
$$

and the integration (with the appropriate choice of integration constants) of this equation, (C.21b), yields

$$
{ }^{(j+1)} \zeta_{n}(\tau)=b_{n}+v_{n}\left(\tau-\tau_{b}\right)+O\left(\left|\left(\tau-\tau_{b}\right)\right|^{1+\gamma}\right), \quad n=3, \ldots, N,
$$

which is clearly consistent with (C.14b). (Note however that the estimate (C.21b) can be replaced by more stringent estimates if $a_{n 1}=a_{n 2}$ and $v_{n} \neq 0$, or if $v_{n}=0$; indeed it is easily seen from (C.21a) that the last term in the right hand sides of (C.14b) and (C.22) can be replaced by $O\left(\left|\tau-\tau_{b}\right|^{2}\right)$ if $a_{n 1}=a_{n 2}$ and $v_{n} \neq 0$, and by $O\left(\left|\tau-\tau_{b}\right|^{2+\gamma},\left|\tau-\tau_{b}\right|^{3-\gamma}\right)$ if $v_{n}=0$. This entails in subsequent equations some corresponding changes that the 
reader will easily ascertain, but which do not modify the main conclusions, see Section 5 , as regards the nature of the branch point of the solution $\underline{\zeta}(\tau)$ ).

This concludes our treatment meant to justify the validity of the representation (5.4).

Let us now proceed to justify the ansatz (5.6), namely the compatibility of the expansion

$$
\begin{aligned}
& \zeta_{1}(\tau)=b+c\left(\tau-\tau_{b}\right)^{1+\beta}+\sum_{k, l=1 ; k+l \geq 3}^{\infty} g_{k l}^{(1)}\left(\tau-\tau_{b}\right)^{k+l \beta}, \\
& \zeta_{2}(\tau)=b+v_{2}\left(\tau-\tau_{b}\right)-c\left(\tau-\tau_{b}\right)^{1+\beta}+\sum_{k=1}^{\infty} \sum_{l=2 \delta_{k 1}}^{\infty} g_{k l}^{(2)}\left(\tau-\tau_{b}\right)^{k+l \beta}, \\
& \zeta_{n}(\tau)=b_{n}+v_{n}\left(\tau-\tau_{b}\right)+\sum_{k=2}^{\infty} \sum_{l=0}^{\infty} g_{k l}^{(n)}\left(\tau-\tau_{b}\right)^{k+l \beta}, \quad n=3, \ldots, N,
\end{aligned}
$$

where

$$
\beta=-2 a, \quad \operatorname{Re}(\beta)>0,
$$

with the evolution equation (1.7) satisfied by the solution $\underline{\zeta}(\tau)$. Of course this ansatz, (C.23), is again only applicable for $\tau \approx \tau_{b}$, namely in the neighborhood of the value $\tau_{b}$ of the independent variable where the solution $\underline{\zeta}(\tau)$ features a "two-body collision", characterized, see (C.23), by the equality and inequalities

$$
\zeta_{1}\left(\tau_{b}\right)=\zeta_{2}\left(\tau_{b}\right) \neq \zeta_{n}\left(\tau_{b}\right), \quad n=3, \ldots, N .
$$

And clearly in that neighborhood this ansatz, (C.23), entails

$$
\begin{aligned}
& \zeta_{1}(\tau)=b+c\left(\tau-\tau_{b}\right)^{1+\beta}+O\left(\left|\tau-\tau_{b}\right|^{2+\beta},\left|\tau-\tau_{b}\right|^{1+2 \beta}\right), \\
& \zeta_{2}(\tau)=b+v_{2}\left(\tau-\tau_{b}\right)-c\left(\tau-\tau_{b}\right)^{1+\beta}+O\left(\left|\tau-\tau_{b}\right|^{2},\left|\tau-\tau_{b}\right|^{1+2 \beta}\right), \\
& \zeta_{n}(\tau)=b_{n}+v_{n}\left(\tau-\tau_{b}\right)+O\left(\left|\tau-\tau_{b}\right|^{2}\right), \quad n=3, \ldots, N .
\end{aligned}
$$

Here we limit our treatment to show that this behavior is compatible with the evolution equation (1.7), which is the key point of our argument (see above - it is instead left for the diligent reader to repeat, in close analogy to what we did before, the iteration argument that leads to the ansatz (C.23)).

Clearly the expansions (C.25) entail

$$
\begin{aligned}
& \zeta_{1}^{\prime}(\tau)=(1+\beta) c\left(\tau-\tau_{b}\right)^{\beta}\left[1+O\left(\left|\tau-\tau_{b}\right|,\left|\tau-\tau_{b}\right|^{\beta}\right)\right], \\
& \zeta_{2}^{\prime}(\tau)=v_{2}\left[1+O\left(\left|\tau-\tau_{b}\right|,\left|\tau-\tau_{b}\right|^{\beta}\right)\right], \\
& \zeta_{n}^{\prime}(\tau)=v_{n}\left[1+O\left(\left|\tau-\tau_{b}\right|\right)\right], \quad n=3, \ldots, N,
\end{aligned}
$$

hence

$$
\begin{aligned}
& \zeta_{1}^{\prime}(\tau) \zeta_{n}^{\prime}(\tau)=(1+\beta) c v_{n}\left(\tau-\tau_{b}\right)^{\beta}\left[1+O\left(\left|\tau-\tau_{b}\right|,\left|\tau-\tau_{b}\right|^{\beta}\right)\right], \\
& n=2, \ldots, N \\
& \zeta_{2}^{\prime}(\tau) \zeta_{n}^{\prime}(\tau)= v_{2} v_{n}\left[1+O\left(\left|\tau-\tau_{b}\right|,\left|\tau-\tau_{b}\right|^{\beta}\right)\right], \quad n=3, \ldots, N, \\
& \zeta_{n}^{\prime}(\tau) \zeta_{m}^{\prime}(\tau)=v_{n} v_{m}\left[1+O\left(\left|\tau-\tau_{b}\right|\right)\right], \quad n, m=3, \ldots, N,
\end{aligned}
$$


as well as

$$
\begin{aligned}
& \zeta_{1}(\tau)-\zeta_{2}(\tau)=-v_{2}\left(\tau-\tau_{b}\right)\left[1+O\left(\left|\tau-\tau_{b}\right|^{1+\beta}\right)\right], \\
& \zeta_{s}(\tau)-\zeta_{n}(\tau)=\left(b-b_{n}\right)\left[1+O\left(\left|\tau-\tau_{b}\right|\right)\right], \quad s=1,2, \quad n=3, \ldots, N, \\
& \zeta_{n}(\tau)-\zeta_{m}(\tau)=\left(b_{n}-b_{m}\right)\left[1+O\left(\left|\tau-\tau_{b}\right|\right)\right], \quad n, m=3, \ldots, N,
\end{aligned}
$$

hence

$$
\begin{aligned}
& {\left[\zeta_{1}(\tau)-\zeta_{2}(\tau)\right]^{-1}=-v_{2}^{-1}\left(\tau-\tau_{b}\right)^{-1}\left[1+O\left(\left|\tau-\tau_{b}\right|^{1+\beta}\right)\right],} \\
& {\left[\zeta_{s}(\tau)-\zeta_{n}(\tau)\right]^{-1}=\left(b-b_{n}\right)^{-1}\left[1+O\left(\left|\tau-\tau_{b}\right|\right)\right],} \\
& \quad s=1,2, \quad n=3, \ldots, N, \\
& {\left[\zeta_{n}(\tau)-\zeta_{m}(\tau)\right]^{-1}=\left(b_{n}-b_{m}\right)^{-1}\left[1+O\left(\left|\tau-\tau_{b}\right|\right)\right], \quad n, m=3, \ldots, N .}
\end{aligned}
$$

It is now easy, using these formulas, to verify the consistency of (C.25) with (1.7). Indeed for $n=1$ (1.7) yields (via (C.27a) and (C.29a,b))

$$
\begin{aligned}
\zeta_{1}^{\prime \prime}= & -2 a c(1+\beta)\left(\tau-\tau_{b}\right)^{-1+\beta} \\
& \times\left[1+O\left(\left|\tau-\tau_{b}\right|,\left|\tau-\tau_{b}\right|^{\beta}\right)\right]+O\left(\left|\tau-\tau_{b}\right|^{\beta}\right),
\end{aligned}
$$

namely, via (C.23d),

$$
\zeta_{1}^{\prime \prime}=c \beta(1+\beta)\left(\tau-\tau_{b}\right)^{-1+\beta}+O\left(\left|\tau-\tau_{b}\right|^{\beta},\left|\tau-\tau_{b}\right|^{-1+2 \beta}\right),
$$

which can be immediately integrated (with an appropriate choice of integration constants) to yield (C.25a). Likewise for $n=2$ (1.7) yields (via (C.27a) and (C.29a,b))

$$
\zeta_{2}^{\prime \prime}=2 a c(1+\beta)\left(\tau-\tau_{b}\right)^{-1+\beta}\left[1+O\left(\left|\tau-\tau_{b}\right|,\left|\tau-\tau_{b}\right|^{\beta}\right)\right]+O(1)
$$

namely, via (C.23d),

$$
\zeta_{2}^{\prime \prime}=-c \beta(1+\beta)\left(\tau-\tau_{b}\right)^{-1+\beta}+O\left(\left|\tau-\tau_{b}\right|^{-1+2 \beta}, 1\right),
$$

which can be immediately integrated (with an appropriate choice of integration constants) to yield (C.25b). And finally for $n=3, \ldots, N$ (1.7) yields (via (C.27b,c) and (C.29b,c))

$$
\zeta_{n}^{\prime \prime}=O(1),
$$

which can be immediately integrated (with an appropriate choice of integration constants) to yield (C.25c).

\section{Appendix D}

In this Appendix D we introduce the monic polynomials $\Pi_{N}(\zeta, \tau)$ respectively $P_{N}(z, t)$, of degree $N$ in the variable $\zeta$ respectively $z$, the zeros of which coincide with the coordinates, 
$\zeta_{n} \equiv \zeta_{n}(\tau)$ respectively $z_{n} \equiv z_{n}(t)$, of the particles moving according to the $N$-body problems (1.7) respectively (1.5),

$$
\begin{aligned}
& \Pi_{N}(\zeta, \tau)=\zeta^{N}+\sum_{m=1}^{N} \gamma_{m}(\tau) \zeta^{N-m}=\prod_{n=1}^{N}\left[\zeta-\zeta_{n}(\tau)\right], \\
& P_{N}(z, t)=z^{N}+\sum_{m=1}^{N} c_{m}(t) z^{N-m}=\prod_{n=1}^{N}\left[z-z_{n}(t)\right] .
\end{aligned}
$$

Via such a position a one-to-one correspondence is introduced among the (ordered) sets of coefficients $\left\{\gamma_{m}(\tau), m=1, \ldots, N\right\}$ respectively $\left\{c_{m}(t), m=1, \ldots, N\right\}$ and the (unordered) sets of zeros $\left\{\zeta_{n}(\tau), n=1, \ldots, N\right\}$ respectively $\left\{z_{n}(t), n=1, \ldots, N\right\}$. This correspondence turns out to be extremely convenient $[5,2]$ in the integrable case in which all the coupling constants $a_{n m}$ in (1.5) and (1.7) equal unity,

$$
a_{n m}=1 \text {. }
$$

Indeed in this integrable case these polynomials satisfy extremely simple linear PDEs, namely (note that throughout this Appendix D subscripted variables denote partial differentiations)

$$
\begin{aligned}
& \Pi_{N, \tau \tau}=0, \\
& P_{N, t t}=i \omega P_{N, t},
\end{aligned}
$$

entailing of course

$$
\begin{aligned}
& \gamma_{m}^{\prime \prime}=0, \quad \gamma_{m}(\tau)=\gamma_{m}(0)+\gamma_{m}^{\prime}(0) \tau \\
& \ddot{c}_{m}=i \omega \dot{c}_{m}, \quad c_{m}(t)=c_{m}(0)+\dot{c}_{m}(0)[\exp (i \omega t)-1] /(i \omega) .
\end{aligned}
$$

It is this connection that lies behind the resolvent formula (1.17).

In this Appendix D we investigate the extent to which an analogous approach is useful to treat the $N$-body problems (1.7) and (1.5) in the (generally nonintegrable) case when the coupling constants $a_{n m}$ are still all equal, but they need not be unity,

$$
a_{n m}=a,
$$

with $a$ an arbitrary (possibly complex) constant.

Hereafter we focus on the $N$-body problem (1.7), and only at the end, by taking advantage of our by now usual trick (see (1.6)), we mention the analogous results related to the $N$-body problem (1.5). We also drop, whenever this is notationally convenient, the subscript $N$ attached to the symbols $\Pi$ and $P$ (namely hereafter $\Pi_{N} \equiv \Pi, P_{N} \equiv P$ ).

Our results hinge on the following basic result, the proof of which is relegated to the very end of this Appendix.

Lemma D.1. If the $N$ zeros $\zeta_{n}(\tau)$ evolve according to (1.7) with (D.6), the (monic) polynomial $\Pi(\zeta, \tau)$ of degree $N$ in $\zeta$, see (D.1), satisfies the following nonlinear PDE:

$$
\Pi_{\tau \tau}\left[\Pi_{\zeta}\right]^{2}+(1-a)\left\{\Pi_{\zeta \zeta}\left(\Pi_{\tau}\right)^{2}-\Pi_{\zeta}\left[\left(\Pi_{\tau}\right)^{2}\right]_{\zeta}\right\}=\Psi \Pi
$$


where $\Psi \equiv \Psi(\zeta, \tau) \equiv \Psi_{2 N-4}(\zeta, \tau)$ is a polynomial of degree $2 N-4$ in the variable $\zeta$,

$$
\Psi(\zeta, \tau)=\sum_{k=0}^{2 N-4} \psi_{k}(\tau) \zeta^{N-k} .
$$

Note that this PDE, (D.7), does not explicitly feature the parameter $N$.

Let us note that (D.1) entails the identification

$$
\gamma_{1}(\tau)=-\sum_{n=1}^{N} \zeta_{n}(\tau),
$$

so that the coefficient $\gamma_{1}(\tau)$ clearly, see (1.7), satisfies the equation

$$
\gamma_{1}^{\prime \prime}(\tau)=0 .
$$

Hence $\Pi_{\tau \tau}(\zeta, \tau)$ is a polynomial in $\zeta$ of degree $N-2$ (see (D.1)). It is therefore clear, see (D.1), that the two sides of the PDE (D.7) are polynomials of degree $3 N-4$ in $\zeta$, hence this PDE entails $3 N-3$ equations to be satisfied by the $N$ coefficients $\gamma_{n}(\tau)$, see (D.1), and the $2 N-3$ coefficients $\psi_{k}(\tau)$, see (D.8). Hence the number of equations matches the number of unknowns $(3 N-3=N+(2 N-3))$. Moreover these equations are clearly all linear and purely algebraic (not differential!) for the $2 N-3$ coefficients $\psi_{k}(\tau)$, see (D.7) and (D.8). It is therefore in principle possible to eliminate the $2 N-3$ unknowns $\psi_{k}(\tau)$ and to thereby obtain a system of only $N$, generally nonlinear, second-order ODEs for the $N$ unknowns $\gamma_{n}(\tau)$, or rather a system of $N-1$, generally nonlinear, second-order ODEs for the $N-1$ unknowns $\gamma_{n}(\tau), n=2, \ldots, N$, since $\gamma_{1}(\tau)$ shall turn out to satisfy the linear second-order ODE (D.10a), which of course entails

$$
\gamma_{1}(\tau)=\gamma_{1}(0)+\gamma_{1}^{\prime}(0) \tau
$$

A systematic algorithmic procedure ("Euclid's method") to eliminate the coefficients of the polynomial $\Psi$ from (D.7) and to thereby obtain the (generally nonlinear) secondorder ODEs satisfied by the coefficients $\gamma_{n}(\tau)$ can be based on the observation (which is actually instrumental, see below, to obtain the PDE (D.7)) that the right-hand side of (D.7) vanishes for $\zeta=\zeta_{n}$, see (D.1). One can therefore lower the degree of the left-hand side of (D.7) by applying sequentially the substitution

$$
\zeta^{N}=-\sum_{m=1}^{N} \gamma_{m} \zeta^{N-m}
$$

to the term of highest degree, or to all terms of degree higher than $N$. After applying this procedure $2 N-3$ times the left-hand side of (D.7) becomes a polynomial in $\zeta$ of degree $N-1$, since each application lowers by one unit the degree of the polynomial. But since the polynomial of degree $N-1$ thus obtained must vanish at the $N$ points $\zeta=\zeta_{n}$ (see (D.7)), it vanishes identically, namely all its $N$ coefficients vanish: and this requirement yields precisely the $N$ equations satisfied by the $N$ coefficients $\gamma_{n}(\tau)$ (hence one of these $N$ equations will be precisely (D.10a)). For instance for $N=2$ one thereby obtains

$$
\gamma_{2}^{\prime \prime}=2(1-a)\left(4 \gamma_{2}-\gamma_{1}^{2}\right)^{-1}\left(\gamma_{2}^{\prime 2}-\gamma_{2}^{\prime} \gamma_{1}^{\prime} \gamma_{1}+\gamma_{1}^{\prime 2} \gamma_{2}\right)
$$


of course with $\gamma_{1}(\tau)$ given by (D.10b), and for $N=3$ one obtains

$$
\begin{aligned}
\gamma_{2}^{\prime \prime}= & 2(1-a) \delta^{-1}\left[\gamma_{1}^{\prime 2}\left(-6 \gamma_{2}^{2} \gamma_{1}^{2}-4 \gamma_{1} \gamma_{2} \gamma_{3}+\gamma_{2}^{3}+9 \gamma_{3}^{2}\right)+\gamma_{2}^{\prime 2}\left(-3 \gamma_{1} \gamma_{3}+\gamma_{2}^{2}\right)\right. \\
& +\gamma_{3}^{\prime 2}\left(\gamma_{1}^{2}-3 \gamma_{2}\right)+\gamma_{1}^{\prime} \gamma_{2}^{\prime}\left(-\gamma_{1} \gamma_{2}^{2}+4 \gamma_{1}^{2} \gamma_{3}-3 \gamma_{2} \gamma_{3}\right)+2 \gamma_{1}^{\prime} \gamma_{3}^{\prime}\left(-3 \gamma_{1} \gamma_{3}+\gamma_{2}^{2}\right) \\
& \left.+\gamma_{2}^{\prime} \gamma_{3}^{\prime}\left(-3 \gamma_{3} \gamma_{1}+\gamma_{2}^{2}\right)\right] \\
\gamma_{3}^{\prime \prime}= & 2(1-a) \delta^{-1}\left[\gamma_{1}^{\prime 2} \gamma_{3}\left(-3 \gamma_{1} \gamma_{3}+\gamma_{2}^{2}\right)+\gamma_{2}^{\prime 2} \gamma_{3}\left(\gamma_{1}^{2}-3 \gamma_{2}\right)+\gamma_{3}^{\prime 2}\left(\gamma_{1}^{3}-4 \gamma_{1} \gamma_{2}+9 \gamma_{3}\right)\right. \\
& +\gamma_{1}^{\prime} \gamma_{2}^{\prime} \gamma_{3}\left(-\gamma_{1} \gamma_{2}+9 \gamma_{3}\right)+2 \gamma_{1}^{\prime} \gamma_{3}^{\prime} \gamma_{3}\left(\gamma_{1}^{2}-3 \gamma_{2}\right) \\
& \left.+\gamma_{2}^{\prime} \gamma_{3}^{\prime}\left(-3 \gamma_{1} \gamma_{3}-\gamma_{1}^{2} \gamma_{2}+4 \gamma_{2}^{2}\right)\right] \\
\delta= & 27 \gamma_{3}^{2}-18 \gamma_{3} \gamma_{2} \gamma_{1}+4 \gamma_{3} \gamma_{1}^{3}+4 \gamma_{2}^{3}-\gamma_{2}^{2} \gamma_{1}^{2},
\end{aligned}
$$

of course with $\gamma_{1}(\tau)$ given again by (D.10b).

Note the consistency of these equations with the general result $\gamma_{n}^{\prime \prime}=0$, valid for arbitrary $N$ in the integrable $(a=1)$ case $[5,2]$.

The diligent reader will also verify the consistency of these ODEs, (D.12), (D.13) and (D.7), with the solutions in the free $(a=0)$ case, when of course, for all $n=1, \ldots, N$ with arbitrary $N, \zeta_{n}(\tau)=\zeta_{n}(0)+\zeta_{n}^{\prime}(0) \tau$ (see (1.7) and (D.6), with $a=0$ ), and the coefficients $\gamma_{n}(\tau)$ have the corresponding expressions entailed by the well-known explicit formulas expressing the coefficients of a polynomial in terms of its zeros, see (D.1). The diligent reader will also obtain the solutions of (D.12) that correspond to the two-body problems treated in Section 4, and the solutions of (D.13) respectively (D.7) that correspond to the similarity solutions (with $N=3$ respectively arbitrary $N$ ) of Section 3.

The results of [11] entail the conjecture that, if $a=-1 / 2$, the solutions of the two nonlinear coupled nonautonomous ODEs (D.13) with (D.10b) possess the ("super Painlevé") property to only feature solutions which are entire functions of the independent variable $\tau$.

Let us now report tersely the analogous results relevant to the equations of motion (1.5) rather than (1.7). To this end we set

$$
\Pi_{N}(\zeta, \tau)=P_{N}(z, t), \quad \zeta=z, \quad \tau=[\exp (i \omega t)-1] /(i \omega)
$$

as suggested by (1.6), and we then note that the nonlinear PDE (D.7) can now be rewritten as follows:

$$
\left(P_{t t}-i \omega P_{t}\right)\left[P_{z}\right]^{2}+(1-a)\left\{P_{z z}\left(P_{t}\right)^{2}-P_{z}\left[\left(P_{t}\right)^{2}\right]_{z}\right\}=Q P .
$$

Here we introduced via

$$
Q \equiv Q(z, t)=\exp (-2 i \omega t) \Psi(\zeta, \tau)
$$

the polynomial $Q(z, t)$ of degree $2 N-4$ in the variable $z$,

$$
Q(z, t)=\sum_{k=0}^{2 N-4} q_{k}(t) z^{N-k} .
$$

We do not elaborate any further on the polynomial solutions of the PDE (D.15), since with trivial modifications what is written above after (D.7) remains applicable, and it is as well quite easy to apply the change of (independent) variable $\gamma_{n}(\tau)=c_{n}(t)$, see 
(D.14) and (D.1,2) in order to obtain from (D.10a), from (D.12) and from (D.13) the corresponding ODEs for the coefficients $c_{n}(t)$. We only note that we expect (D.15) to possess generally a lot of solutions that are completely periodic with period $T$, see (1.2), in the (real) independent variable $t$. And in particular it is natural (see above) to conjecture that, if $a=-1 / 2$, all polynomial solutions $P(z, t)$ of degree $N=3$ of the nonlinear PDE (D.15) are completely periodic with period $T$ in the (real) independent variable $t$.

Finally, let us obtain (D.7) from (D.1) and (1.7) with (D.6).

Differentiation of (D.1) with respect to $\tau$ yields

$$
\sum_{m=1}^{N} \gamma_{m}^{\prime}(\tau) \zeta^{N-m}=-\sum_{n=1}^{N} \zeta_{n}^{\prime}(\tau) \prod_{l=1 ; l \neq n}^{N}\left[\zeta-\zeta_{l}(\tau)\right]
$$

and a further differentiation yields

$$
\begin{aligned}
\sum_{m=1}^{N} \gamma_{m}^{\prime \prime}(\tau) \zeta^{N-m} & =-\sum_{n=1}^{N} \zeta_{n}^{\prime \prime}(\tau) \prod_{l=1 ; l \neq n}^{N}\left[\zeta-\zeta_{l}(\tau)\right] \\
& +\sum_{n=1}^{N} \zeta_{n}^{\prime}(\tau) \sum_{l=1 ; l \neq n}^{N} \zeta_{l}^{\prime}(\tau) \prod_{k=1 ; k \neq l, n}^{N}\left[\zeta-\zeta_{k}(\tau)\right] .
\end{aligned}
$$

We now set $\zeta=\zeta_{n}$ in (D.18a) and we thereby get

$$
\zeta_{n}^{\prime}(\tau)=-\sum_{m=1}^{N} \gamma_{m}^{\prime}(\tau)\left[\zeta_{n}(\tau)\right]^{N-m} / \Pi_{\zeta}\left[\zeta_{n}(\tau), \tau\right],
$$

where we used the relation

$$
\Pi_{\zeta}\left[\zeta_{n}(\tau), \tau\right] \equiv \partial \Pi(\zeta, \tau) /\left.\partial \zeta\right|_{\zeta=\zeta_{n}(\tau)}=\prod_{l=1 ; l \neq n}^{N}\left[\zeta_{n}(\tau)-\zeta_{l}(\tau)\right] .
$$

Likewise, by setting $\zeta=\zeta_{n}$ in (D.18b) we get

$$
\begin{array}{r}
-\zeta_{n}^{\prime \prime}(\tau)+2 \zeta_{n}^{\prime}(\tau) \sum_{l=1 ; l \neq n}^{N} \zeta_{l}^{\prime}(\tau) /\left[\zeta_{n}(\tau)-\zeta_{l}(\tau)\right] \\
=\sum_{m=1}^{N} \gamma_{m}^{\prime \prime}(\tau)\left[\zeta_{n}(\tau)\right]^{N-m} / \Pi_{\zeta}\left[\zeta_{n}(\tau), \tau\right],
\end{array}
$$

where we used again (D.20).

We now use the equations of motion (1.7) and we thereby get

$$
\begin{aligned}
2 \zeta_{n}^{\prime}(\tau) & \sum_{l=1 ; l \neq n}^{N}\left(1-a_{n l}\right) \zeta_{l}^{\prime}(\tau) /\left[\zeta_{n}(\tau)-\zeta_{l}(\tau)\right] \\
= & \sum_{m=1}^{N} \gamma_{m}^{\prime \prime}(\tau)\left[\zeta_{n}(\tau)\right]^{N-m} / \Pi_{\zeta}\left[\zeta_{n}(\tau), \tau\right],
\end{aligned}
$$


and via (D.19a) this yields

$$
\begin{aligned}
& 2 \sum_{m=1}^{N} \gamma_{m}^{\prime}(\tau)\left[\zeta_{n}(\tau)\right]^{N-m} \sum_{l=1 ; l \neq n}^{N}\left(1-a_{n l}\right) \sum_{k=1}^{N} \gamma_{k}^{\prime}(\tau)\left[\zeta_{l}(\tau)\right]^{N-k} \\
& \quad \times\left\{\left[\zeta_{n}(\tau)-\zeta_{l}(\tau)\right] \Pi_{\zeta}\left[\zeta_{l}(\tau), \tau\right]\right\}^{-1}=\sum_{m=1}^{N} \gamma_{m}^{\prime \prime}(\tau)\left[\zeta_{n}(\tau)\right]^{N-m}
\end{aligned}
$$

From now on we restrict consideration to the equal-coupling-constants case, see (D.6), and we re-write (D.23a) as follows:

$$
\begin{aligned}
& 2 \sum_{m, k=1}^{N} \gamma_{m}^{\prime}(\tau) \gamma_{k}^{\prime}(\tau)\left[\zeta_{n}(\tau)\right]^{N-m} \sum_{l=1 ; l \neq n}^{N}(1-a)\left[\zeta_{l}(\tau)\right]^{N-k} \\
& \quad \times\left\{\left[\zeta_{n}(\tau)-\zeta_{l}(\tau)\right] \Pi_{\zeta}\left[\zeta_{l}(\tau), \tau\right]\right\}^{-1}=\sum_{m=1}^{N} \gamma_{m}^{\prime \prime}(\tau)\left[\zeta_{n}(\tau)\right]^{N-m} .
\end{aligned}
$$

We now note that the rational function of $\zeta$,

$$
F_{n l}(\zeta)=\zeta^{N-k}\left[\left(\zeta_{n}-\zeta\right) \Pi(\zeta)\right]^{-1},
$$

where $\Pi(\zeta)$ is the polynomial of degree $N$ that has the $N$ zeros $\zeta_{n}$ (see (D.1)) and $k$ is an arbitrary positive integer not larger than $N, k=1, \ldots, N$, vanishes faster than $\zeta^{-1}$ as $\zeta \rightarrow \infty$, so that the sum of all its residues vanishes. Since this rational function has $N-1$ simple poles (at $\zeta=\zeta_{m}, m=1, \ldots, N, m \neq n$ ) and a double pole (at $\zeta=\zeta_{n}$ ), this implies the identity

$$
\begin{aligned}
& \sum_{l=1 ; l \neq n}^{N} \zeta_{l}^{N-k}\left[\left(\zeta_{n}-\zeta_{l}\right) \Pi_{\zeta}\left(\zeta_{l}\right)\right]^{-1} \\
& \quad=(N-k) \zeta_{n}^{N-k-1}\left[\Pi_{\zeta}\left(\zeta_{n}\right)\right]^{-1}-(1 / 2) \zeta_{n}^{N-k} \Pi_{\zeta \zeta}\left(\zeta_{n}\right)\left[\Pi_{\zeta}\left(\zeta_{n}\right)\right]^{-2}
\end{aligned}
$$

Via this identity (D.23b) yields

$$
\begin{aligned}
& (1-a) \sum_{m, k=1}^{N} \gamma_{m}^{\prime}(\tau) \gamma_{k}^{\prime}(\tau)\left\{2(N-k)\left[\zeta_{n}(\tau)\right]^{2 N-m-k-1} \Pi_{\zeta}\left[\zeta_{n}(\tau), \tau\right]\right. \\
& \left.\quad-\left[\zeta_{n}(\tau)\right]^{2 N-m-k} \Pi_{\zeta \zeta}\left[\zeta_{n}(\tau), \tau\right]\right\}=\left(\Pi_{\zeta}\left[\zeta_{n}(\tau), \tau\right]\right)^{2} \sum_{m=1}^{N} \gamma_{m}^{\prime \prime}(\tau)\left[\zeta_{n}(\tau)\right]^{N-m} .
\end{aligned}
$$

We now note that the definition (D.1) of the polynomial $\Pi(\zeta, \tau)$ implies the relations

$$
\begin{aligned}
& \sum_{m, k=1}^{N} \gamma_{m}^{\prime}(\tau) \gamma_{k}^{\prime}(\tau)\left[\zeta_{n}(\tau)\right]^{2 N-m-k}=\left.\left[\Pi_{\tau}(\zeta, \tau)\right]^{2}\right|_{\zeta=\zeta_{n}(\tau)}, \\
& \sum_{m, k=1}^{N} \gamma_{m}^{\prime}(\tau) \gamma_{k}^{\prime}(\tau) 2(N-k)\left[\zeta_{n}(\tau)\right]^{2 N-m-k-1} \\
& \quad=\sum_{m, k=1}^{N} \gamma_{m}^{\prime}(\tau) \gamma_{k}^{\prime}(\tau)(2 N-m-k)\left[\zeta_{n}(\tau)\right]^{2 N-m-k-1}=\left.\left\{\left[\Pi_{\tau}(\zeta, \tau)\right]^{2}\right\}_{\zeta}\right|_{\zeta=\zeta_{n}(\tau)}
\end{aligned}
$$


where the first step in (D.27b) is of course justified by the possibility to exchange the dummy indices $m$ and $k$. Hence from (D.26) and (D.1) we infer that the polynomial

$$
(1-a)\left\{\Pi_{\zeta}\left(\left[\Pi_{\tau}\right]^{2}\right)_{\zeta}-\Pi_{\zeta \zeta}\left[\Pi_{\tau}\right]^{2}\right\}-\Pi_{\tau \tau}\left[\Pi_{\zeta}\right]^{2}
$$

of degree $3 N-4$ in $\zeta$, vanishes at the $N$ points $\zeta=\zeta_{n}$, namely that it has the same zeros as the polynomial of $N$ degree $\Pi$, see (D.1), and this clearly implies the validity of (D.7).

\section{References}

[1] Calogero F and Françoise J-P, Periodic Solutions of a Many-Rotator Problem in the Plane, Inverse Problems 17 (2001), 1-8.

[2] Calogero F, Classical Many-Body Problems Amenable to Exact Treatments, Lecture Notes in Physics Monograph m 66, Springer, 2001.

[3] Calogero F, A Class of Integrable Hamiltonian Systems whose Solutions are (Perhaps) All Completely Periodic, J. Math. Phys. 38 (1997), 5711-5719.

[4] Calogero F, Tricks of the Trade: Relating and Deriving Solvable and Integrable Dynamical Systems, in Calogero-Moser-Sutherland Models, Editors: van Diejen J F and Vinet L, Proceedings of the Workshop on Calogero-Moser-Sutherland Models held in Montreal, 10-15 March 1997, CRM Series in Mathematical Physics, Springer, 2000, 93-116.

[5] Calogero F, Motion of Poles and Zeros of Special Solutions of Nonlinear and Linear Partial Differential Equations, and Related 'Solvable' Many-Body Problems, Nuovo Cimento B43 (1978), 177-241.

[6] Erdélyi A (Editor), Higher Transcendental Functions, McGraw-Hill, 1953.

[7] Ince E L, Ordinary Differential Equations, Dover, 1956.

[8] Sommacal M, Studio analitico e numerico di sistemi a molti corpi, Dissertation to obtain the degree of "Dottore in Fisica", Università di Roma "La Sapienza", 2002.

[9] Calogero F and Sommacal M, Periodic Solutions of a System of Complex ODEs. II. Higher Periods, J. Nonlin. Math. Phys. 9 (2002), 483-516.

[10] Calogero F, Periodic Solutions of a System of Complex ODEs, Phys. Lett. A293 (2002), $146-150$.

[11] Calogero F, Solvable Three-Body Problem and Painlevé Conjectures, Theor. Math. Phys. 133 (2002), 1443-1452; Erratum 134 (2003), 139.

[12] Calogero F, The "Neatest" Many-Body Problem Amenable to Exact Treatments (a "Goldfish"?), Physica D 152/153 (2001), 78-84. 